\title{
Recent Developments in Wintgen Inequality and Wintgen Ideal Submanifolds
}

\author{
Bang-Yen Chen \\ (Dedicated to the memory of Prof. Dr. Aurel BEJANCU (1946 - 2020))
}

\begin{abstract}
P. Wintgen proved in [Sur l'inégalité de Chen-Willmore. C. R. Acad. Sci. Paris 288, 993-995 (1979)] that the Gauss curvature $G$ and the normal curvature $K^{D}$ of a surface in the Euclidean 4-space $\mathbb{E}^{4}$ satisfy

$$
G+\left|K^{D}\right| \leq\|H\|^{2},
$$

where $\|H\|^{2}$ is the squared mean curvature. A surface $M^{2}$ in $\mathbb{E}^{4}$ is called a Wintgen ideal surface if it satisfies the equality case of the inequality identically. Wintgen ideal surfaces in $\mathbb{E}^{4}$ form an important family of surfaces; namely, surfaces with circular ellipse of curvature. In 1999, P. J. De Smet, F. Dillen, L. Verstraelen and L. Vrancken gave a conjecture for Wintgen inequality on Riemannian submanifolds in real space forms, which was well-known as the DDVV conjecture. Later, the DDVV conjecture was proven by $\mathrm{Z}$. Lu and by Ge and $\mathrm{Z}$. Tang independently.

In this paper, we provide a comprehensive survey on recent developments in Wintgen inequality and Wintgen ideal submanifolds.
\end{abstract}

\section{Contents}

1 Introduction

2 Preliminaries 9

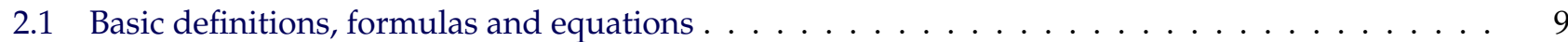

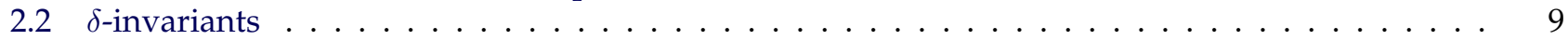

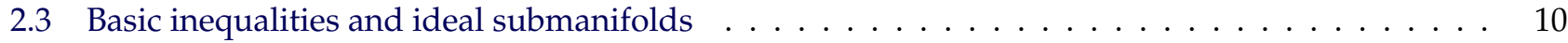

2.4 Hopf fibration and Lagrangian submanifolds of complex space forms . . . . . . . . . . . . . 10

3 Wintgen's inequality and Wintgen ideal surfaces in $\mathbb{E}^{4} \quad 11$

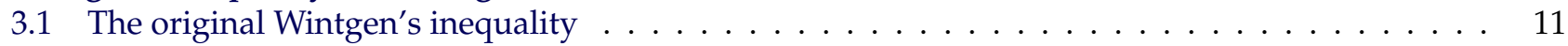

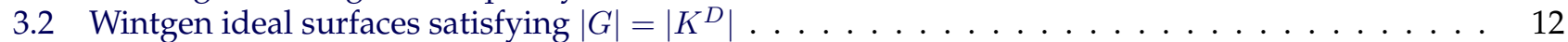

3.3 Wintgen's inequality for surfaces in real space forms $\ldots \ldots \ldots \ldots \ldots \ldots$

4 Wintgen type inequality for surfaces in indefinite real space form $R_{2}^{4}(c) \quad 14$

4.1 Pseudo-Riemannian space forms . . . . . . . . . . . . . . . . . . . . . . . 14

4.2 Wintgen type inequality for space-like surfaces in $R_{2}^{4}(c) \ldots \ldots \ldots \ldots \ldots \ldots \ldots$

4.3 Wintgen ideal surfaces in $\mathbb{E}_{2}^{4}$ satisfying $|G|=\left|K^{D}\right| \ldots \ldots \ldots \ldots \ldots \ldots$

4.4 Wintgen ideal surfaces in $\mathbb{E}_{2}^{4}$ satisfying $K^{D}=2 G \ldots \ldots \ldots \ldots \ldots \ldots \ldots$ 
5 Complex curves and space-like surfaces in $\mathbb{E}_{2}^{4}$

5.1 Complex curves and space-like surfaces satisfying $K^{D}=-G$ in $\mathbb{E}_{2}^{4} \ldots \ldots \ldots \ldots$

5.2 Space-like surfaces with constant Gauss curvature and null normal curvature . . . . . . . . . 16

6 Space-like minimal surfaces in $R_{2}^{4}(c) \quad 16$

6.1 Space-like minimal surfaces in $R_{2}^{4}(c) \ldots \ldots \ldots \ldots \ldots \ldots \ldots \ldots \ldots \ldots$

6.2 Applications to minimal Wintgen ideal surfaces in $R_{2}^{4}(c) \ldots \ldots \ldots \ldots \ldots \ldots \ldots$

7 DDVV conjecture and theorem 18

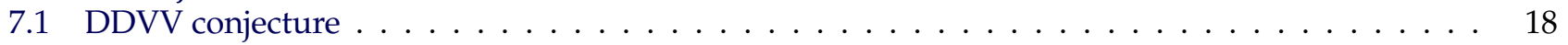

7.2 The Solution of DDVV conjecture by Z. Lu, J. Ge and Z. Tang . . . . . . . . . . . . . . . 19

8 Some properties of Wintgen ideal submanifolds 19

8.1 Wintgen ideal submanifolds of real space forms are Chen submanifolds . . . . . . . . . . . 20

8.2 Ricci and Casorati principal directions of Wintgen ideal submanifolds . . . . . . . . . . . . . 20

8.3 Some classification theorems for Wintgen ideal submanifolds . . . . . . . . . . . . . . . . . . 20

9 Wintgen ideal submanifolds in Kaehler manifolds 21

9.1 Normal scalar curvature for complex submanifolds . . . . . . . . . . . . . . . . . . . . . . . 21

9.2 Wintgen inequality for totally and Lagrangian submanifolds . . . . . . . . . . . . . . . . . 22

9.3 Wintgen inequality for slant and CR-submanifolds . . . . . . . . . . . . . . . . . 22

9.4 Bi-slant submanifolds in locally conformal Kaehler space forms . . . . . . . . . . . . . . . 23

10 Wintgen ideal submanifolds in Sasakian space forms 23

10.1 Almost contact metric manifold and Sasakian space forms . . . . . . . . . . . . . . 23

10.2 Invariant submanifolds in Sasakian space forms . . . . . . . . . . . . . . . . . . . . 24

$10.3 C$-totally real, Legendrian and slant submanifolds in Sasakian space forms . . . . . . . . . . . 24

$10.4 C$-totally real, Legendrian and bi-slant submanifolds in Kenmotsu space forms . . . . . . . . . 25

10.5 Legendrian submanifolds in generalized Sasakian-space-forms . . . . . . . . . . . . . . . . 26

11 Wintgen ideal submanifolds in quaternionic space forms 26

11.1 Quaternionic Kaehler manifold and quaternionic space forms . . . . . . . . . . . . . . 26

11.2 Lagrangian and slant submanifolds in quaternionic space forms . . . . . . . . . . . . . 27

11.3 Wintgen ideal inequality for quaternionic CR-submanifolds . . . . . . . . . . . . . . . . . 27

12 Wintgen ideal submanifolds in golden Riemannian space forms 28

12.1 Locally Golden product space forms . . . . . . . . . . . . . . . . . . . . . . . . . . . . . 29

$12.2 C$-totally real submanifolds of a locally Golden product space form . . . . . . . . . . . . . 29

12.3 Slant and invariant submanifolds of a locally Golden product space form . . . . . . . . . . . . . 29

13 Wintgen ideal submanifolds for statistical submanifolds 30

13.1 Statistical manifolds . . . . . . . . . . . . . . . . . . . . . . . 30

13.2 Statistical submanifolds in statistical manifolds . . . . . . . . . . . . . . . . . . 31

13.3 Wintgen type inequalities of statistical submanifolds in statistical space forms . . . . . . . . . 32

13.4 Totally real statistical submanifolds in holomorphic statistical space forms . . . . . . . . . . . 32

13.5 Statistical submanifolds of statistical manifolds with quasi-constant curvature . . . . . . . . . 33

13.6 Wintgen type inequalities for submanifolds in statistical warped products . . . . . . . . . . 33

13.7 Wintgen type inequality for Legendrian submanifolds of Sasakian statistical manifolds _ . . . . 33

13.8 Statistical submanifolds of Kenmotsu statistical manifolds . . . . . . . . . . . . . . . . . . . . . 34

13.9 Legendrian submanifolds of almost Kenmotsu statistical manifolds . . . . . . . . . . . . . . 35

14 Möbious geometry and Wintgen ideal submanifolds 36

14.1 Submanifold theory in Möbius geometry . . . . . . . . . . . . . . . . . 36

14.2 Wintgen ideal submanifolds with a two-dimensional integrable distribution . . . . . . . . . . . 37

14.3 Mean curvature spheres and Wintgen ideal submanifolds of codimension two . . . . . . . . . 37

14.4 Möbius homogenous surfaces in $S^{4} \ldots \ldots \ldots \ldots \ldots \ldots \ldots$

14.5 Wintgen ideal submanifolds with vanishing Möbius form . . . . . . . . . . . . . . . 39

14.6 Conformal Gauss map and Möbius geometry of Wintgen ideal submanifolds . . . . . . . . . 39 
14.7 Classification of Wintgen ideal submanifolds via Möbius geometry ～. . . . . . . . . . . . . 39

15 Symmetry of Wintgen ideal submanfiolds 40

15.1 Deszcz's pseudo-symmetry . . . . . . . . . . . . . . . . . . . . . . . . . 40

15.2 Pseudo-symmetry of Wintgen ideal submanifolds in real space forms . . . . . . . . . . . . . . 41

15.3 Pseudo-psymmetry of Wintgen ideal Lagrangian submanifolds in complex space forms . . . . . 41

15.4 Pseudo-psymmetry of Wintgen ideal Legendrian submanifolds in Sasakian space forms . . . . . 41

15.5 Roter spaces, Deszcz symmetric spaces, and Wintgen ideal submanifolds . . . . . . . . . . . . 42

References

$37-40$

\section{Introduction}

For a surface $M^{2}$ in a Euclidean 3-space $\mathbb{E}^{3}$, the well-known Euler inequality in classical differential geometry is given by

$$
G \leq\|H\|^{2},
$$

where $G$ is the Gauss curvature of $M^{2}$ and $\|H\|^{2}$ is the squared mean curvature of $M^{2}$ in $\mathbb{E}^{3}$. Obviously, $G=\|H\|^{2}$ everywhere on $M$ if and only if the surface $M^{2}$ is totally umbilical in $\mathbb{E}^{3}$.

For an isometric immersion $\psi: M^{2} \rightarrow \widetilde{M}^{4}$ of a surface $M$ into a Riemannian 4-manifold $\widetilde{M}^{4}$, the ellipse of curvature at a point $p \in M^{2}$ is defined as

$$
\mathcal{E}_{p}=\left\{h(X, X) \mid X \in T_{p} M^{2},\|X\|=1\right\},
$$

where $h$ denotes the second fundamental form of $M^{2}$ in $\widetilde{M}^{4}$. The ellipse of curvature is the analogue of the Dupin indicatrix of an ordinary surface in $\mathbb{E}^{3}$.

A surface $\psi: M^{2} \rightarrow \widetilde{M}^{4}$ is called super-minimal if and only if, at each point $p \in M^{2}$, the ellipse of curvature $\mathcal{E}_{p}$ is a circle with center 0 (see [74]). Simple examples of super-minimal surfaces in the Euclidean 4-space $\mathbb{E}^{4}$ are $R$-surfaces, i.e., graphs of holomorphic functions: $\{(z, f(z)): z \in U\}$, where $U \subset \mathbb{C} \approx \mathbf{R}^{2}$ is an open subset of the complex plane and $f$ is a holomorphic function.

Th. Friedrich proved in 1984 that super-minimal surfaces are characterized by the property that the lift into the twistor space is holomorphic and horizontal (see [74, 75] for details). When the ambient space $\widetilde{M}^{4}$ is a space of constant curvature, O. Borůvka [18] proved in 1928 that the family of super-minimal immersions $\psi: M^{2} \rightarrow \tilde{M}^{4}$ depends (locally) on two holomorphic functions. In [20], R. Bryant proved that every compact Riemann surface admits a conformal super-minimal immersion into the ordinary 4-sphere $S^{4}$. M. Dajczer and R. Tojeiro established in [59] a representation formula for super-minimal surfaces in $\mathbb{E}^{4}$ in terms of pairs $(g, h)$ of conjugate minimal surfaces in $\mathbb{E}^{4}$. For further results on super-minimal surfaces we refer to $\S 2$ of my survey article [41].

In 1979, P. Wintgen [137] proved a basic relationship between the intrinsic Gauss curvature G, the extrinsic normal curvature $K^{D}$, and squared mean curvature $\|H\|^{2}$ of any surface $M^{2}$ in a Euclidean 4-space $\mathbb{E}^{4}$; namely,

$$
G+\left|K^{D}\right| \leq\|H\|^{2},
$$

with the equality holding if and only if the curvature ellipse is a circle. Wintgen's inequality was extended in [83] by I. V. Guadalupe and L. Rodriguez to surfaces of arbitrary codimension in real space forms $R^{2+m}(c)$ with $m \geq 2$. Also, the present author extended Wintgen's inequality in $[38,42]$ to surfaces in pseudo-Euclidean 4 -space $\mathbb{E}_{2}^{4}$ with a neutral metric.

In 1999, P. J. De Smet, F. Dillen, L. Verstraelen and L. Vrancken proposed in [61] a conjecture of Wintgen inequality for general Riemannian submanifolds in real space forms, which was later well-known as the DDVV conjecture. This conjecture was proven to be true by Z. Lu [94] and by Ge and Z. Tang [78] independently. Since then, the study of Wintgen's inequalities and Wintgen ideal submanifolds has attracting more and more researchers and a lot of interesting results have been achieved during the last 15 years.

The main purpose of this paper is to provide a comprehensive survey on recent developments in Wintgen inequality and Wintgen ideal submanifolds done mainly in the last 15 years. 


\section{Preliminaries}

We follow the notations from $[40,45,46,109]$.

\subsection{Basic definitions, formulas and equations}

Let $\psi: M^{n} \rightarrow \widetilde{M}^{m}$ be an isometric immersion of an $n$-dimensional Riemannian manifold $M^{n}$ into a Riemannian $m$-manifold $\widetilde{M}^{m}$. Denote by $\nabla$ and $\widetilde{\nabla}$ the Levi-Civita connections on $M^{n}$ and $\widetilde{M}^{m}$, respectively, and by $\langle$,$\rangle the inner product on M^{n}$ and $\tilde{M}^{m}$. Let $R$ and $\widetilde{R}$ denote the Riemann curvature tensors of $M^{n}$ and $\widetilde{M}^{m}$, respectively, so that we have

$$
R(X, Y) Z=\nabla_{X} \nabla_{Y}-\nabla_{Y} \nabla_{X} Z-\nabla_{[X, Y]} Z .
$$

For vector fields $X, Y$ tangent to $M^{n}$ and $\xi$ normal to $M^{n}$, the formulas of Gauss and Weingarten are given respectively by

$$
\begin{aligned}
& \widetilde{\nabla}_{X} Y=\nabla_{X} Y+h(X, Y), \\
& \widetilde{\nabla}_{X} \xi=-A_{\xi} X+D_{X} \xi,
\end{aligned}
$$

where $h, A$ and $D$ are the second fundamental form $h$, the shape operator $A$, and the normal connection $D$ of $M^{n}$ in $\widetilde{M}^{m}$. The shape operator and the second fundamental form are related by

$$
\langle h(X, Y), \xi\rangle=\left\langle A_{\xi} X, Y\right\rangle .
$$

The mean curvature vector is defined by $\vec{H}=\frac{1}{n}$ trace $h$. The squared mean curvature $\|H\|^{2}$ is defined to be $\langle\vec{H}, \vec{H}\rangle$.

For a submanifold $M^{n}$ of a Riemannian $m$-manifold $\widetilde{M}^{m}$, we denote by $\left(h_{i j}^{r}\right)$ the coefficients of the second fundamental form defined by

$$
h_{i j}^{r}=\left\langle h\left(e_{i}, e_{j}\right), \xi_{r}\right\rangle,
$$

where $\left\{e_{1}, \ldots, e_{n}\right\}$ and $\left\{\xi_{1}, \ldots, \xi_{m-n}\right\}$ are is an orthonormal frames of the tangent bundle $T M^{n}$ and normal bundle $T^{\perp} M^{n}$, respectively.

The equations of Gauss, Codazzi and Ricci of $M^{n}$ in $\widetilde{M}^{m}$ are given respectively by

$$
\begin{aligned}
& \langle R(X, Y), Z, W\rangle=\langle\widetilde{R}(X, Y) Z, W\rangle+\langle h(X, W), h(Y, Z)\rangle-\langle h(X, Z), h(Y, W)\rangle, \\
& (\widetilde{R}(X, Y) Z)^{\perp}=\left(\bar{\nabla}_{X} h\right)(Y, Z)-\left(\bar{\nabla}_{Y} h\right)(X, Z), \\
& \left\langle R^{D}(X, Y) \xi, \eta\right\rangle=\langle\widetilde{R}(X, Y) \xi, \eta\rangle+\left\langle\left[A_{\xi}, A_{\eta}\right] X, Y\right\rangle
\end{aligned}
$$

for vector fields $X, Y, Z$ tangent to $M$ and $\xi, \eta$ normal to $M$, where $(\widetilde{R}(X, Y) Z)^{\perp}$ is the normal component of $\widetilde{R}(X, Y) Z, \bar{\nabla} h$ is defined by

$$
\left(\bar{\nabla}_{X} h\right)(Y, Z)=D_{X} h(Y, Z)-h\left(\nabla_{X} Y, Z\right)-h\left(Y, \nabla_{X} Z\right)
$$

and $R^{D}$ is the curvature tensor associated with the normal connection $D$, i.e.,

$$
R^{D}(X, Y) \xi=D_{X} D_{Y} \xi-D_{Y} D_{X} \xi-D_{[X, Y]} \xi
$$

For a surface $M^{2}$ in $\mathbb{E}^{4}$, the Gauss curvature $G$ and the normal curvature $K^{D}$ of $M^{2}$ are given by

$$
G=\left\langle R^{D}\left(e_{1}, e_{2}\right) e_{2}, e_{1}\right\rangle, \quad K^{D}=\left\langle R^{D}\left(e_{1}, e_{2}\right) \xi_{2}, \xi_{1}\right\rangle .
$$

\section{2. $\delta$-invariants}

Let $M^{n}$ be a Riemannian $n$-manifold and a point $p \in M^{n}$. Denote by $K(\pi)$ the sectional curvature of $M^{n}$ associated with 2-plane section $\pi \subset T_{p} M^{n}$. For an orthonormal basis $\left\{e_{1}, \ldots, e_{n}\right\}$ of $T_{p} M^{n}$, the scalar curvature $\tau$ at $p$ is defined by

$$
\tau(p)=\sum_{i<j} K\left(e_{i} \wedge e_{j}\right) .
$$


If $L$ is an $r$-dimensional subspace of $T_{p} M^{n}$ with $r \geq 2$ and if $\left\{e_{1}, \ldots, e_{r}\right\}$ is an orthonormal basis of $L$, then the scalar curvature $\tau(L)$ of $L$ is defined by

$$
\tau(L)=\sum_{\alpha<\beta} K\left(e_{\alpha} \wedge e_{\beta}\right), \quad 1 \leq \alpha, \beta \leq r .
$$

For two given integers $n \geq 3$ and $k \geq 1$, we denote by $\mathcal{S}(n, k)$ the finite set consisting of all $k$-tuples $\left(n_{1}, \ldots, n_{k}\right)$ of integers satisfying $\sum_{i=1}^{k} n_{i} \leq n$ with $2 \leq n_{i}<n$. We put $\mathcal{S}(n)=\cup_{k \geq 1} \mathcal{S}(n, k)$.

For each $\left(n_{1}, \ldots, n_{k}\right) \in \mathcal{S}(n)$ and each $p \in M^{n}$, the author introduced the notion of $\delta$-invariant $\delta\left(n_{1}, \ldots, n_{k}\right)(p)$ in $[25,30,32]$ by

$$
\delta\left(n_{1}, \ldots, n_{k}\right)(p)=\tau(p)-\inf \left\{\tau\left(L_{1}\right)+\cdots+\tau\left(L_{k}\right)\right\},
$$

where $L_{1}, \ldots, L_{k}$ run over all $k$ mutually orthogonal subspaces of $T_{p} M^{n}$ such that $\operatorname{dim} L_{j}=n_{j}, j=1, \ldots, k$.

\subsection{Basic inequalities and ideal submanifolds}

The author proved the following optimal universal inequality in [30,32] which relates the $\delta$-invariants $\delta\left(n_{1}, \ldots, n_{k}\right)$ and the squared mean curvature $\|H\|^{2}$ for submanifolds in real space forms.

Theorem 2.1. Let $M^{n}$ be an n-dimensional submanifold of a real space form $R^{m}(c)$ of constant sectional curvature $c$. Then, for each $k$-tuple $\left(n_{1}, \ldots, n_{k}\right) \in \mathcal{S}(n)$, we have

$$
\delta\left(n_{1}, \ldots, n_{k}\right) \leq \frac{n^{2}\left(n+k-1-\sum n_{j}\right)}{2\left(n+k-\sum n_{j}\right)}\|H\|^{2}+\frac{1}{2}\left(n(n-1)-\sum_{j=1}^{k} n_{j}\left(n_{j}-1\right)\right) c .
$$

The equality case of inequality (2.12) holds at a point $x \in M^{n}$ if and only if there exists an orthonormal basis $\left\{e_{1}, \ldots, e_{m}\right\}$ at $x$ such that the shape operators of $M^{n}$ in $R^{m}(c)$ at $x$ with respect to $\left\{e_{1}, \ldots, e_{m}\right\}$ take the following form:

$$
A_{r}=\left(\begin{array}{cccc}
A_{1}^{r} & \ldots & 0 & \\
\vdots & \ddots & \vdots & 0 \\
0 & \ldots & A_{k}^{r} & \\
& 0 & & \mu_{r} I
\end{array}\right), \quad r=n+1, \ldots, m
$$

where $I$ is an identity matrix and $A_{j}^{r}$ is a symmetric $n_{j} \times n_{j}$ submatrix satisfying

$$
\operatorname{trace}\left(A_{1}^{r}\right)=\cdots=\operatorname{trace}\left(A_{k}^{r}\right)=\mu_{r} .
$$

The notions of ideal immersions and ideal submanifolds were introduced in the 1990s by the author in [30] as follows. An $n$-dimensional submanifold $M^{n}$ of a real space form $R^{m}(c)$ is said to be $\delta\left(n_{1}, \ldots, n_{k}\right)$-ideal if it satisfies the equality case of inequality (2.12) identically. And a submanifold $M^{n}$ in $R^{m}(c)$ is simply called an ideal submanifold if it is a $\delta\left(n_{1}, \ldots, n_{k}\right)$-ideal for some $\left(n_{1}, \ldots, n_{k}\right) \in \mathcal{S}(n)$.

Physical Interpretation of Ideal Immersions. "An isometric immersion $\phi: M^{n} \rightarrow R^{m}(c)$ of a Riemannian $n$ manifold into a real space form $R^{m}(c)$ is an ideal immersion" means that $M^{n}$ receives the least possible amount of tension (given by $\hat{\Delta}_{0}(p)$ ) from the surrounding space at each point in $M^{n}$. This is due to inequality (2.12) and the well-known fact that the mean curvature vector field is exactly the tension field for isometric immersions. For an isometric immersion, the squared mean curvature at each point on the submanifold simply measures the amount of tension the submanifold receiving from the surrounding space at that point. For this reason, sometime an ideal immersion is also called a best way of living (see, [30, 32, 33, 35, 40] for details).

\subsection{Hopf fibration and Lagrangian submanifolds of complex space forms}

We recall the Hopf fibration and the general method of H. Reckziegel [114] for constructing Lagrangian submanifolds in complex projective spaces and in complex hyperbolic spaces.

Case (1): $C P^{n}(4)$. Let $S^{2 n+1}(c)=\left\{Z=\left(z_{1}, \ldots, z_{n+1}\right) \in \mathbb{C}^{n+1}:\langle Z, Z\rangle=\frac{1}{c}>0\right\}$ be the hypersphere of $\mathbb{C}^{n+1}$ with constant sectional curvature $c$ centered at the origin. Consider the complex structure $J$ induced by $i=\sqrt{-1}$ and 
the inner product $\langle$,$\rangle on \mathbb{C}^{n+1}$. Let us consider the canonical Sasakian structure on $S^{2 n+1}(1)$ consisting of $\phi$ given by the projection of the complex structure $J$ of $\mathbb{C}^{n+1}$ on the tangent bundle of $S^{2 n+1}(1)$ and the structure vector field $\xi=J x$ with $x$ being the position vector.

An isometric immersion $f: M^{n} \rightarrow S^{2 n+1}(1)$ is called $C$-totally real (or Legendrian) if $\xi$ is normal to $f_{*}\left(T M^{n}\right.$ ) and $\left\langle J\left(f_{*}\left(T M^{n}\right)\right), f_{*}\left(T M^{n}\right)\right\rangle=0$, where $J$ denotes the canonical complex structure of $\mathbb{C}^{n+1}$. Since vectors of $S^{2 n+1}(1)$ perpendicular to $\xi$ at a point $Z$ define the horizontal subspace $\mathcal{H}_{z}$ of the Hopf fibration

$$
\pi: S^{2 n+1}(1) \rightarrow C P^{n}(4),
$$

the condition " $\xi$ is normal to $f_{*}\left(T M^{n}\right)$ " means that $f$ is horizontal.

The main results of [114] can be specialized as follows. Let $f: M^{n} \rightarrow C P^{n}(4)$ be a Lagrangian isometric immersion. Then there exists an isometric covering map $\tau: \hat{M} \rightarrow M^{n}$, and a $C$-totally real isometric immersion $\hat{f}: \hat{M} \rightarrow S^{2 n+1}(1)$ such that $f(\tau)=\pi(\hat{f})$. Hence every Lagrangian immersion can be lifted locally (or globally if we assume the manifold is simply connected) to a $C$-totally real immersion of the same Riemannian manifold.

Conversely, let $f: \hat{M} \rightarrow S^{2 n+1}(1)$ be a $C$-totally real isometric immersion. Then $f=\pi(\hat{f}): M^{n} \rightarrow C P^{n}(4)$ is again an isometric immersion, which is Lagrangian. Under this correspondence, the second fundamental forms $h^{\hat{f}}$ and $h^{f}$ of $\hat{f}$ and $f$ satisfy $\pi_{*}\left(h^{\hat{f}}\right)=h^{f}$. Moreover, $h^{\hat{f}}$ is horizontal with respect to $\pi$.

Case (2): $C H^{n}(-4)$. We consider the complex number space $\mathbb{C}_{1}^{n+1}$ with the pseudo-Euclidean metric:

$$
g_{0}=-d z_{1} d \bar{z}_{1}+\sum_{j=2}^{n+1} d z_{j} d \bar{z}_{j} .
$$

Put $H_{1}^{2 n+1}(c)=\left\{Z=\left(z_{1}, z_{2}, \ldots, z_{n+1}\right):\langle Z, Z\rangle=\frac{1}{c}<0\right\}$, where $\langle$,$\rangle is the inner product on \mathbf{C}_{1}^{n+1}$ induced from $g_{0}$. We put $T_{z}^{\prime}=\left\{Z \in \mathbb{C}^{n+1}: \operatorname{Re}\langle U, Z\rangle=\operatorname{Re}\langle U, i Z\rangle=0\right\}$ and $H_{1}^{1}=\{\lambda \in \mathbf{C}: \lambda \bar{\lambda}=1\}$. Then we have an $H_{1}^{1}$-action on $H_{1}^{2 n+1}(-1), Z \mapsto \lambda Z$ and at each point $Z \in H_{1}^{2 n+1}(-1)$, the vector $i Z$ is tangent to the flow of the action. Since the metric $g_{0}$ is Hermitian, we have $\operatorname{Re} g_{0}(i Z, i Z)=-1$. Note that the orbit is given by $x_{t}=(\cos t+i \sin t) z$ and $d x_{t} / d t=i Z_{t}$. Thus the orbit lies in the negative definite plane spanned by $Z$ and $i Z$. The quotient space $H_{1}^{2 n+1} / \sim$, under the identification from the action, is the complex hyperbolic space $C H^{n}(-4)$ with constant holomorphic sectional curvature -4 , with the complex structure $J$ induced from the canonical complex structure $J$ on $\mathbb{C}_{1}^{n+1}$ via the following Hopf fibration:

$$
\pi: H_{1}^{2 n+1}(-1) \rightarrow C H^{n}(-4) .
$$

Just as in Case (1), let $f: M^{n} \rightarrow C H^{n}(-4)$ be a Lagrangian isometric immersion. Then there exists an isometric covering map $\tau: \hat{M} \rightarrow M^{n}$, and a $C$-totally real isometric immersion $\hat{f}: \hat{M} \rightarrow H_{1}^{2 n+1}(-1)$ such that $f(\tau)=\pi(\hat{f})$. Hence every totally real immersion can be lifted locally (or globally if we assume the manifold is simply connected) to a $C$-totally real immersion.

Conversely, let $\hat{f}: \hat{M} \rightarrow H_{1}^{2 n+1}(-1)$ be a $C$-totally real isometric immersion. Then $f=\pi(\hat{f}): M^{n} \rightarrow C H^{n}(-4)$ is again an isometric immersion, which is Lagrangian. Similarly, under this correspondence, the second fundamental forms $h^{\hat{f}}$ and $h^{f}$ of $\hat{f}$ and $f$ satisfy $\pi_{*}\left(h^{\hat{f}}\right)=h^{f}$. Moreover, $h^{\hat{f}}$ is horizontal with respect to $\pi$.

\section{Wintgen's inequality and Wintgen ideal surfaces in $\mathbb{E}^{4}$}

\subsection{The original Wintgen's inequality}

The result of P. Wintgen obtained in [137] is the following.

Theorem 3.1. Let $M^{2}$ be a surface in Euclidean 4-space $\mathbb{E}^{4}$. Then we have

$$
\|H\|^{2} \geq G+\left|K^{D}\right|
$$

at any point $x \in M^{2}$. Moreover,

(i) If $K^{D} \geq 0$ holds at a point $x \in M^{2}$, then the equality sign of (3.1) holds at $x$ if and only if, with respect to some suitable orthonormal basis $\left\{e_{1}, e_{2}, e_{3}, e_{4}\right\}$ at $x$, the shape operators at $x$ satisfies

$$
A_{e_{3}}=\left(\begin{array}{cc}
\mu+2 \gamma & 0 \\
0 & \mu
\end{array}\right), A_{e_{4}}=\left(\begin{array}{ll}
0 & \gamma \\
\gamma & 0
\end{array}\right)
$$


(ii) If $K^{D}<0$ holds at $x \in M^{2}$, then the equality sign of (3.1) holds at $x$ if and only if, with respect to some suitable orthonormal basis $\left\{e_{1}, e_{2}, e_{3}, e_{4}\right\}$ at $x$, the shape operators at $x$ satisfies

$$
A_{e_{3}}=\left(\begin{array}{cc}
\mu-2 \gamma & 0 \\
0 & \mu
\end{array}\right), A_{e_{4}}=\left(\begin{array}{ll}
0 & \gamma \\
\gamma & 0
\end{array}\right) .
$$

Analogous to ideal submanifolds defined in $§ 2$, a surface $M^{2}$ in $\mathbb{E}^{4}$ is called Wintgen ideal if it satisfies the equality case of the Wintgen inequality (3.1) identically.

The following are some simple examples of Wintgen ideal surfaces in $\mathbb{E}^{4}$.

Example 3.1. Consider the Whitney sphere in the complex Euclidean plane $\mathbf{C}^{2}$ defined by

$$
w\left(y_{0}, y_{1}, y_{2}\right)=\frac{1+\mathrm{i} y_{0}}{1+y_{0}^{2}}\left(y_{1}, y_{2}\right)
$$

with $y_{0}^{2}+y_{1}^{2}+y_{2}^{2}=1$. The Whitney immersion $w: S^{2} \rightarrow \mathbf{C}^{2}$ is a Lagrangian immersion of a sphere $S^{2}$ into $\mathbf{C}^{2}$ which has a unique self-intersection point at $w(-1,0,0)=w(1,0,0)$. The Whitney sphere satisfies the equality case of (3.1) identically.

Example 3.2. The rotation surface of Vrănceanu is defined by the immersion $f: \mathbf{R} \times(0,2 \pi) \rightarrow \mathbb{E}^{4}$,

$$
f(u, v)=r(u)(\cos u \cos v, \cos u \sin v, \sin u \cos v, \sin u \sin v),
$$

where $r>0$ is a smooth real-valued function. The ellipse of curvature of the rotation surface of Vrănceanu given by (3.5) is a circle if and only if either

$$
\begin{gathered}
r(u)=\frac{A}{\sqrt{|\cos (2 u+a)|}}, \quad A>0, a \in \mathbf{R}, \text { or } \\
r(u)=B \sqrt{|\cos (2 u+b)|}, \quad B>0, b \in \mathbf{R} .
\end{gathered}
$$

In the first case the rotation surface of Vrănceanu is the tensor product of an equilateral hyperbola and a unit circle and in the second case the tensor product of a lemniscate of Bernoulli and a unit circle (for the definition of tensor products of immersions, see [26, 62]).

\subsection{Wintgen ideal surfaces satisfying $|G|=\left|K^{D}\right|$}

The following result was given in [36].

Proposition 3.1. Let $M^{2}$ be a Wintgen ideal surface in $\mathbb{E}^{4}$. Then $M^{2}$ has constant mean curvature and constant Gauss curvature if and only if $M^{2}$ is totally umbilical.

The next theorem classifies all Wintgen ideal surfaces in $\mathbb{E}^{4}$ with equal Gauss and normal curvatures.

Theorem 3.2. [36] A Wintgen ideal surface $M^{2}$ in $\mathbb{E}^{4}$ satisfies $|K|=\left|K^{D}\right|$ identically if and only if one of the following four cases occurs:

(1) $M^{2}$ is an open portion of a totally geodesic plane in $\mathbb{E}^{4}$.

(2) $M^{2}$ is a complex curve lying fully in $\mathbb{C}^{2}$, where $\mathbb{C}^{2}$ is the Euclidean 4-space $\mathbb{E}^{4}$ endowed with some orthogonal almost complex structure.

(3) Up to dilations and rigid motions on the Euclidean 4-space $\mathbb{E}^{4}, M^{2}$ is an open portion of the Whitney sphere defined by

$$
\psi(u, v)=\frac{\sin u}{1+\cos ^{2} u}(\sin v, \cos v, \cos u \sin v, \cos u \cos v) .
$$


(4) Up to dilations and rigid motions of the Euclidean 4-space $\mathbb{E}^{4}, M^{2}$ is a surface with $K=K^{D}=\frac{1}{2}\|H\|^{2}$ defined by

$$
\begin{aligned}
\psi(x, y)= & \frac{2 \sqrt{y}}{5} \sqrt{\cos x} \cos \left(\frac{x}{2}\right) \cos (\ln y) \cos \left(\frac{1}{2} \tanh ^{-1}\left(\tan \frac{x}{2}\right)\right) \\
\times & \left(\tan \left(\frac{1}{2} \tanh ^{-1}\left(\tan \frac{x}{2}\right)\right)(2-\tan (\ln y))+\tan \left(\frac{x}{2}\right)(1+2 \tan (\ln y)),\right. \\
& \tan \left(\frac{1}{2} \tanh ^{-1}\left(\tan \frac{x}{2}\right)\right)(1+2 \tan (\ln y))-\tan \left(\frac{x}{2}\right)(2-\tan (\ln y)), \\
& \tan \left(\frac{x}{2}\right) \tan \left(\frac{1}{2} \tanh ^{-1}\left(\tan \frac{x}{2}\right)\right)(1+2 \tan (\ln y))+\tan (\ln y)-2, \\
& \left.\tan \left(\frac{x}{2}\right) \tan \left(\frac{1}{2} \tanh ^{-1}\left(\tan \frac{x}{2}\right)\right)(\tan (\ln y)-2)-2 \tan (\ln y)-1\right) .
\end{aligned}
$$

Remark 3.1. To prove Theorem 3.2, the author had solved the following fourth order differential equation:

$$
p^{(4)}(x)-2(\tan x) p^{\prime \prime \prime}(x)+\left(1+\frac{5}{8} \sec ^{2} x\right) p^{\prime \prime}(x)+\left(\frac{5}{8} \sec ^{2} x-2\right)(\tan x) p^{\prime}(x)+\frac{185}{256}\left(\sec ^{4} x\right) p(x)=0
$$

to obtain its exact solution:

$$
\begin{aligned}
p(x)= & \sqrt{\cos x}\left\{\left(c_{1} \cos \left(\frac{x}{2}\right)+c_{2} \sin \left(\frac{x}{2}\right)\right) \cos \left(\frac{1}{2} \tanh ^{-1}\left(\tan \left(\frac{x}{2}\right)\right)\right)\right. \\
& \left.+\left(c_{3} \cos \left(\frac{x}{2}\right)+c_{4} \sin \left(\frac{x}{2}\right)\right) \sin \left(\frac{1}{2} \tanh ^{-1}\left(\tan \left(\frac{x}{2}\right)\right)\right)\right\}
\end{aligned}
$$

Remark 3.2. It was proved by I. Castro in [22] that, up to rigid motions and dilations of $\mathbb{C}^{2}$, the Whitney sphere is the only compact orientable Lagrangian super-minimal surface in $\mathbb{C}^{2}$.

\subsection{Wintgen's inequality for surfaces in real space forms}

I. V. Guadalupe and L. Rodriguez [83] used ellipse of curvature to define the normal curvature $K^{D}$ for oriented compact surfaces into oriented real space forms $R^{m}(c)$ of constant curvature $c$. Further, they proved the following.

Theorem 3.3. Let $\phi: M^{2} \rightarrow R^{m}(c)$ be an isometric immersion of a compact oriented surface $M^{2}$ into an orientable $m$-dimensional real space form $R^{m}(c)$ of constant curvature $c$. Then we have the following

$$
\int_{M^{2}}\|H\|^{2} d v+c \operatorname{Area}\left(M^{2}\right) \geq 2 \pi \chi(M)+\left|\int_{M^{2}} K^{D} d v\right|
$$

with equality if and only if $K^{D}$ does not change sign and the ellipse of curvature is a circle at every point. If in addition $M^{2}$ is homeomorphic to the 2-sphere $S^{2}, m=4$ and the mean curvature vector is parallel, then

$$
\left(\|H\|^{2}+c\right) \operatorname{Area}\left(M^{2}\right)=2 \pi\left(\chi\left(M^{2}\right)+\left|\chi\left(T^{\perp} M^{2}\right)\right|\right),
$$

where $\chi\left(M^{2}\right)$ and $\chi\left(T^{\perp} M^{2}\right)$ denote the Euler number of the $M^{2}$ and the normal bundle $T^{\perp} M^{2}$ of $M^{2}$.

A submanifold $M^{n}$ of a Riemannian manifold $\widetilde{M}^{m}$ is called semi-parallel if it satisfies $\bar{R} \cdot h=0$, where $\bar{R}$ is the curvature tensor of the van der Waerden-Bortolotti connection and $h$ is the second fundamental form of $M^{n}$. In [16], B. Bulca, K. Arslan studied the class of semi-parallel Wintgen ideal surfaces in $\mathbb{E}^{m}$ and showed that such surfaces are totally umbilical in $\mathbb{E}^{m}$. 


\section{Wintgen type inequality for surfaces in indefinite real space form $R_{2}^{4}(c)$}

\subsection{Pseudo-Riemannian space forms}

Denote by $\mathbb{E}_{t}^{m}$ the pseudo-Euclidean $m$-space equipped with pseudo-Euclidean metric of index $t$ given by

$$
g_{t}=-\sum_{i=1}^{t} d x_{i}^{2}+\sum_{j=t+1}^{n} d x_{j}^{2}
$$

where $\left(x_{1}, \ldots, x_{m}\right)$ is a rectangular coordinate system of $\mathbb{E}_{t}^{m}$. Let us put

$$
S_{s}^{k}(c)=\left\{x \in \mathbb{E}_{s}^{k+1}:\langle x, x\rangle=\frac{1}{c}>0\right\}, \quad H_{s}^{k}(c)=\left\{x \in \mathbb{E}_{s+1}^{k+1}:\langle x, x\rangle=\frac{1}{c}<0\right\}
$$

where $\langle$,$\rangle is the inner product associated with g_{t}$. It is well known that $S_{s}^{k}(c)$ and $H_{s}^{k}(c)$ are pseudoRiemannian manifolds of constant curvature $c$ and with index $s$, which are known as a pseudo-Riemannian $k$-sphere and a pseudo-hyperbolic $k$-space, respectively. The pseudo-Riemannian manifolds $\mathbb{E}_{s}^{k}, S_{s}^{k}(c)$ and $H_{s}^{k}(c)$ are called indefinite real space forms, simply denoted by $R_{s}^{k}(c)$ (see, e.g., $\left.[40,45,109]\right)$.

A vector $v$ is called space-like (resp., time-like) if $\langle v, v\rangle>0$ (resp., $\langle v, v\rangle<0$ ). A vector $v$ is called light-like if it is nonzero and it satisfies $\langle v, v\rangle=0$. Further, a submanifold $M^{n}$ in a pseudo-Riemannian manifold is called space-like if each nonzero tangent vector of $M^{n}$ is a space-like vector.

An $n$-dimensional space-like submanifold $M^{n}$ of a pseudo-Riemannian manifold is called a Chen submanifold (see, e.g., $[79,80,116])$ if

$$
\sum_{i, j}\left\langle h\left(e_{i}, e_{j}\right), \vec{H}\right\rangle h\left(e_{i}, e_{j}\right)
$$

is parallel to the mean curvature vector $\vec{H}$, where $h$ is the second fundamental form and $\left\{e_{1}, \ldots, e_{n}\right\}$ is an orthonormal frame of the submanifold $M^{n}$.

\subsection{Wintgen type inequality for space-like surfaces in $R_{2}^{4}(c)$}

For oriented space-like surfaces in a 4-dimensional indefinite real space form $R_{2}^{4}(c)$ with neutral metric, the author proved the following Wintgen type inequality in [38, 42].

Theorem 4.1. Let $M^{2}$ be an oriented space-like surface in a 4-dimensional indefinite space form $R_{2}^{4}(c)$ of constant curvature $c$ and with index two. Then we have

$$
G+K^{D} \geq\|H\|^{2}+c
$$

at every point.

The equality sign of (4.4) holds at a point $x \in M^{2}$ if and only if, with respect to some suitable orthonormal frame $\left\{e_{1}, e_{2}, e_{3}, e_{4}\right\}$, the shape operator at $x$ satisfies

$$
A_{e_{3}}=\left(\begin{array}{cc}
\mu+2 \gamma & 0 \\
0 & \mu
\end{array}\right), A_{e_{4}}=\left(\begin{array}{ll}
0 & \gamma \\
\gamma & 0
\end{array}\right)
$$

Similar as before, we call a space-like surface $M^{2}$ in $R_{2}^{4}(c)$ a Wintgen ideal surface if it satisfies the equality case of (4.4) identically.

Remark 4.1. Clearly, every totally geodesic space-like surface in $R_{2}^{4}(c)$ is a Wintgen ideal surface. Furthermore, it follows from (4.3) and (4.5) that every Wintgen ideal surface surface in $R_{2}^{4}(c)$ is a Chen surface.

\subsection{Wintgen ideal surfaces in $\mathbb{E}_{2}^{4}$ satisfying $|G|=\left|K^{D}\right|$}

The following classification theorem was proved in [42].

Theorem 4.2. Let $M^{2}$ be a Wintgen ideal surface in a neutral pseudo-Euclidean 4-space $\mathbb{E}_{2}^{4}$. Then $M^{2}$ satisfies $|G|=\left|K^{D}\right|$ identically if and only if, up to dilations and rigid motions, $M^{2}$ is one of the following three types of surfaces: (i) A space-like complex curve in $\mathbf{C}_{1}^{2}$, where $\mathbf{C}_{1}^{2}$ denotes $\mathbb{E}_{2}^{4}$ endowed with some orthogonal complex structure; 
(ii) An open portion of a non-minimal surface defined by

$$
\sec ^{2} x\left(\sin x \sinh y, \sqrt{2-\sin ^{2} x} \cosh y, \sin x \cosh y, \sqrt{2-\sin ^{2} x} \sinh y\right) ;
$$

(iii) An open portion of a non-minimal surface defined by

$$
\begin{gathered}
\frac{\cosh x}{6 \sqrt{2 y}}\left(6 \sqrt{2} \sqrt{\sqrt{2}+(1-2 \tanh x) \sqrt{1+\tanh x}}+y^{2} \sqrt{\sqrt{2}+\sqrt{1+\tanh x}}, 6 \sqrt{2} \sqrt{\sqrt{2}+(2 \tanh x-1) \sqrt{1+\tanh x}}\right. \\
+y^{2} \sqrt{\sqrt{2}+\sqrt{1+\tanh x}}\left(\sqrt{2} \cosh x \sqrt{1+\tanh x}-e^{x}\right), \\
6 \sqrt{2} \sqrt{\sqrt{2}+(1-2 \tanh x) \sqrt{1+\tanh x}}-y^{2} \sqrt{\sqrt{2}+\sqrt{1+\tanh x}}, 6 \sqrt{2} \sqrt{\sqrt{2}+(2 \tanh x-1) \sqrt{1+\tanh x}} \\
\left.-y^{2} \sqrt{\sqrt{2}+\sqrt{1+\tanh x}}\left(\sqrt{2} \cosh x \sqrt{1+\tanh x}-e^{x}\right)\right) .
\end{gathered}
$$

4.4. Wintgen ideal surfaces in $\mathbb{E}_{2}^{4}$ satisfying $K^{D}=2 G$

The following result was proved by the author and B. D. Suceavă in [52].

Theorem 4.3. For a real number $c$, let $\gamma$ with $3 \gamma^{2}>-c$ be a positive solution of the following second order partial differential equation

$$
\begin{aligned}
& \frac{\partial}{\partial x}\left(\frac{\left(3 \gamma \sqrt{c+3 \gamma^{2}}-c\right)\left(6 \gamma+2 \sqrt{3 c+9 \gamma^{2}}\right)^{\sqrt{3}} \gamma_{x}}{2 \gamma\left(c+3 \gamma^{2}\right)}\right) \\
& \quad-\frac{\partial}{\partial y}\left(\frac{\left(3 \gamma \sqrt{c+3 \gamma^{2}}-c\right) \gamma_{y}}{2 \gamma\left(c+3 \gamma^{2}\right)\left(6 \gamma+2 \sqrt{3 c+9 \gamma^{2}}\right)^{\sqrt{3}}}\right)=\gamma \sqrt{c+3 \gamma^{2}}
\end{aligned}
$$

defined on a simply-connected domain $D \subset \mathbf{R}^{2}$. Then $M_{\gamma}^{2}=\left(D, g_{\gamma}\right)$ with the metric

$$
g_{\gamma}=\frac{\sqrt{c+3 \gamma^{2}}}{\gamma\left(6 \gamma+2 \sqrt{3 c+9 \gamma^{2}}\right)^{\sqrt{3}}}\left(d x^{2}+\left(6 \gamma+2 \sqrt{3 c+9 \gamma^{2}}\right)^{2 \sqrt{3}} d y^{2}\right)
$$

admits a non-minimal Wintgen ideal immersion $\psi_{\gamma}: M_{\gamma}^{2} \rightarrow R_{2}^{4}(c)$ into a complete simply-connected indefinite space form $R_{2}^{4}(c)$ satisfying $K^{D}=2 G$ identically.

The author and Suceavă [52] also proved the following result which classifies all Wintgen ideal surfaces in $R_{2}^{4}(c)$ satisfying $K^{D}=2 G$.

Theorem 4.4. Let $M^{2}$ be a Wintgen ideal surface in a complete simply-connected indefinite space form $R_{2}^{4}(c)$ with $c=1,0$ or -1 . If $M^{2}$ satisfies $K^{D}=2 K$ identically, then one of following three cases occurs:

(1) $c=0$ and $M^{2}$ is a totally geodesic surface in $\mathbb{E}_{2}^{4}$;

(2) $c=-1$ and $M^{2}$ is a minimal surface in $H_{2}^{4}(-1)$ congruent to an open part of $\psi_{\mathcal{B}}:\|H\|^{2}\left(-\frac{1}{3}\right) \rightarrow H_{2}^{4}(-1) \subset \mathbb{E}_{3}^{5}$ defined by

$$
\begin{gathered}
\left(\sinh \left(\frac{2 s}{\sqrt{3}}\right)-\frac{t^{2}}{3}-\left(\frac{7}{8}+\frac{t^{4}}{18}\right) e^{\frac{2 s}{\sqrt{3}}}, t+\left(\frac{t^{3}}{3}-\frac{t}{4}\right) e^{\frac{2 s}{\sqrt{3}}}, \frac{1}{2}+\frac{t^{2}}{2} e^{\frac{2 s}{\sqrt{3}}}, t+\left(\frac{t^{3}}{3}+\frac{t}{4}\right) e^{\frac{2 s}{\sqrt{3}}}\right. \\
\left.\sinh \left(\frac{2 s}{\sqrt{3}}\right)-\frac{t^{2}}{3}-\left(\frac{1}{8}+\frac{t^{4}}{18}\right) e^{\frac{2 s}{\sqrt{3}}}\right)
\end{gathered}
$$

(3) $M^{2}$ is a non-minimal surface in $R_{2}^{4}(c)$ which is congruent to an open part of $\psi_{\gamma}: M_{\gamma}^{2} \rightarrow R_{2}^{4}(c)$ associated with a positive solution $\gamma$ of the partial differential equation (4.6) as described in Theorem 4.3. 


\section{Complex curves and space-like surfaces in $\mathbb{E}_{2}^{4}$}

\subsection{Complex curves and space-like surfaces satisfying $K^{D}=-G$ in $\mathbb{E}_{2}^{4}$}

Lorentzian minimal surfaces in a pseudo-Euclidean space $\mathbb{E}_{s}^{m}$ was completely classified by the author in [39] and by $\mathrm{H}$. Anciux in [4] independently as follows.

Theorem 5.1. A Lorentzian surface in a pseudo-Euclidean $m$-space $\mathbb{E}_{s}^{m}$ is minimal if and only if locally the immersion takes the form

$$
L(x, y)=z(x)+w(y)
$$

where $z$ and $w$ are null curves satisfying $\left\langle z^{\prime}(x), w^{\prime}(y)\right\rangle \neq 0$.

For space-like minimal surface in $\mathbf{C}_{1}^{2}$, the author proved the following result in [42] which provides a very simple link between complex curves and space-like minimal surfaces satisfying $K^{D}=-G$ in $\mathbf{C}_{1}^{2}$.

Proposition 5.1. Let $M^{2}$ be an oriented space-like minimal surface in $\mathbb{E}_{2}^{4}$. Then $M^{2}$ is a complex curve in a Lorentzian complex plane $\mathbf{C}_{1}^{2}$ (i.e. $\mathbb{E}_{2}^{4}$ equipped with a compatible orthogonal complex structure) if and only if $M^{2}$ satisfies $K^{D}=-G$ identically.

\subsection{Space-like surfaces with constant Gauss curvature and null normal curvature}

The next theorem of the author and Suceavă from [52] classifies some space-like surfaces with null normal curvature in $\mathbb{E}_{2}^{4}$.

Theorem 5.2. Let $M$ be a space-like surface in the pseudo-Euclidean 4-space $\mathbb{E}_{2}^{4}$. If $M$ has constant mean and Gauss curvatures and null normal curvature, then $M$ is congruent to an open part of one of the following six types of surfaces:

(1) A totally geodesic plane in $\mathbb{E}_{2}^{4}$ defined by $(0,0, x, y)$;

(2) a totally umbilical hyperbolic plane $H^{2}\left(-\frac{1}{a^{2}}\right) \subset \mathbb{E}_{1}^{3} \subset \mathbb{E}_{2}^{4}$ given by $(0, a \cosh u, a \sinh u \cos v, a \sinh u \sin v)$, where a is a positive number;

(3) A flat surface in $\mathbb{E}_{2}^{4}$ defined by $\frac{1}{\sqrt{2} k}(\cosh (\sqrt{2} k x), \cosh (\sqrt{2} k y), \sinh (\sqrt{2} k x), \sinh (\sqrt{2} k y))$ where $k$ is a positive number;

(4) A flat surface in $\mathbb{E}_{2}^{4}$ defined by $\left(0, \frac{1}{a} \cosh (a x), \frac{1}{a} \sinh (a x), y\right)$, where a is a positive number;

(5) A flat surface in $\mathbb{E}_{2}^{4}$ defined by

$$
\left(\frac{\cosh (\sqrt{2} x)}{\sqrt{2 k r}}, \frac{\cosh (\sqrt{2} y)}{\sqrt{2 k(2 k-r)}}, \frac{\sinh (\sqrt{2} x)}{\sqrt{2 k r}}, \frac{\sinh (\sqrt{2} y)}{\sqrt{2 k(2 k-r)}}\right),
$$

where $k$ and $r$ are positive numbers satisfying $2 k>r>0$;

(6) A surface of negative curvature $-b^{2}$ in $\mathbb{E}_{2}^{4}$ defined by

$$
\begin{aligned}
\left(\frac{1}{b} \cosh (b x) \cosh (b y), \int_{0}^{y} \cosh (b y) \sinh \left(\frac{4 \sqrt{k^{2}-b^{2}}}{b} \tan ^{-1}\left(\tanh \frac{b y}{2}\right)\right) d y\right. \\
\left.\frac{1}{b} \sinh (b x) \cosh (b y), \int_{0}^{y} \cosh (b y) \cosh \left(\frac{4 \sqrt{k^{2}-b^{2}}}{b} \tan ^{-1}\left(\tanh \frac{b y}{2}\right)\right) d y\right),
\end{aligned}
$$

where $b$ and $k$ are real numbers satisfying $0<b<k$.

\section{Space-like minimal surfaces in $R_{2}^{4}(c)$}

6.1. Space-like minimal surfaces in $R_{2}^{4}(c)$

The following lemma follows immediately from the equation of Gauss.

Lemma 6.1. Let $M^{2}$ be a space-like minimal surface in $R_{2}^{4}(c)$. Then we have $G \geq c$. In particular, if $G=c$ holds identically, then $M$ is totally geodesic. 
For space-like minimal surfaces $M^{2}$ in $R_{2}^{4}(c)$, it follows from [117, Theorem 1] that $M^{2}$ has constant Gauss curvature if and only if it has constant normal curvature.

We recall the following result of M. Sasaki from [117].

Theorem 6.1. Let $M^{2}$ be a space-like minimal surface in $R_{2}^{4}(c)$. If $M^{2}$ has constant Gauss curvature $G$, then either (1) $G=c$ and $M^{2}$ is a totally geodesic surface in $R_{2}^{4}(c)$;

(2) $c<0, G=0$ and $M^{2}$ is locally congruent to the minimal surface defined by $\frac{1}{\sqrt{2}}(\cosh u, \cosh v, 0, \sinh u$, $\sinh v)$, or

(3) $c<0, G=c / 3$ and $M^{2}$ is isotropic.

Now, consider a space-like minimal surface in $H_{2}^{4}(-1)$ as follows. Let $\mathbf{R}^{2}$ be a plane with coordinates $s, t$. Consider a map $\mathcal{B}: \mathbf{R}^{2} \rightarrow \mathbb{E}_{3}^{5}$ given by

$$
\begin{gathered}
\mathcal{B}(s, t)=\left(\sinh \left(\frac{2 s}{\sqrt{3}}\right)-\frac{t^{2}}{3}-\left(\frac{7}{8}+\frac{t^{4}}{18}\right) e^{\frac{2 s}{\sqrt{3}}}, t+\left(\frac{t^{3}}{3}-\frac{t}{4}\right) e^{\frac{2 s}{\sqrt{3}}}, \frac{1}{2}+\frac{t^{2}}{2} e^{\frac{2 s}{\sqrt{3}}}, t+\left(\frac{t^{3}}{3}+\frac{t}{4}\right) e^{\frac{2 s}{\sqrt{3}}},\right. \\
\left.\sinh \left(\frac{2 s}{\sqrt{3}}\right)-\frac{t^{2}}{3}-\left(\frac{1}{8}+\frac{t^{4}}{18}\right) e^{\frac{2 s}{\sqrt{3}}}\right) .
\end{gathered}
$$

The author proved in [37] that the map $\mathcal{B}$ defines a full isometric parallel immersion

$$
\psi_{\mathcal{B}}: H^{2}\left(-\frac{1}{3}\right) \rightarrow H_{2}^{4}(-1)
$$

of the hyperbolic plane $H^{2}\left(-\frac{1}{3}\right)$ of curvature $-\frac{1}{3}$ into the pseudo-hyperbolic 4-space $H_{2}^{4}(-1)$.

The next result was also obtained by the author in [37].

Theorem 6.2. Let $\psi: M^{2} \rightarrow H_{2}^{4}(-1)$ be a parallel full immersion of a space-like surface $M^{2}$ into $H_{2}^{4}(-1)$. Then $M^{2}$ is minimal in $H_{2}^{4}(-1)$ if and only if $M^{2}$ is locally congruent to the surface defined by

$$
\begin{gathered}
\left(\sinh \left(\frac{2 s}{\sqrt{3}}\right)-\frac{t^{2}}{3}-\left(\frac{7}{8}+\frac{t^{4}}{18}\right) e^{\frac{2 s}{\sqrt{3}}}, t+\left(\frac{t^{3}}{3}-\frac{t}{4}\right) e^{\frac{2 s}{\sqrt{3}}}, \frac{1}{2}+\frac{t^{2}}{2} e^{\frac{2 s}{\sqrt{3}}}, t+\left(\frac{t^{3}}{3}+\frac{t}{4}\right) e^{\frac{2 s}{\sqrt{3}}},\right. \\
\left.\sinh \left(\frac{2 s}{\sqrt{3}}\right)-\frac{t^{2}}{3}-\left(\frac{1}{8}+\frac{t^{4}}{18}\right) e^{\frac{2 s}{\sqrt{3}}}\right) .
\end{gathered}
$$

By combining Theorem 6.1 and Theorem 6.2, we obtain the following (see [37]).

Theorem 6.3. Let $M^{2}$ be a non-totally geodesic space-like minimal surface in $H_{2}^{4}(-1)$. If $M^{2}$ has constant Gauss curvature $K$, then either

(1) $G=0$ and $M^{2}$ is congruent to an open part of the surface defined by $\frac{1}{\sqrt{2}}(\cosh u, \cosh v, 0, \sinh u, \sinh v)$, or

(2) $G=-\frac{1}{3}$ and $M^{2}$ is is congruent to an open part of the surface defined by

$$
\begin{gathered}
\left(\sinh \left(\frac{2 s}{\sqrt{3}}\right)-\frac{t^{2}}{3}-\left(\frac{7}{8}+\frac{t^{4}}{18}\right) e^{\frac{2 s}{\sqrt{3}}}, t+\left(\frac{t^{3}}{3}-\frac{t}{4}\right) e^{\frac{2 s}{\sqrt{3}}}, \frac{1}{2}+\frac{t^{2}}{2} e^{\frac{2 s}{\sqrt{3}}}, t+\left(\frac{t^{3}}{3}+\frac{t}{4}\right) e^{\frac{2 s}{\sqrt{3}}}\right. \\
\left.\sinh \left(\frac{2 s}{\sqrt{3}}\right)-\frac{t^{2}}{3}-\left(\frac{1}{8}+\frac{t^{4}}{18}\right) e^{\frac{2 s}{\sqrt{3}}}\right) .
\end{gathered}
$$

\subsection{Applications to minimal Wintgen ideal surfaces in $R_{2}^{4}(c)$}

A function $f$ on a space-like surface $M^{2}$ is called logarithm-harmonic, if $\Delta(\ln f)=0$ holds identically on $M^{2}$, where $\Delta$ is the Laplacian. A function $f$ on $M^{2}$ is called subharmonic if $\Delta f \geq 0$ holds everywhere on $M^{2}$.

The author proved the following results.

Theorem 6.4. [38] Let $\psi: M^{2} \rightarrow H_{2}^{4}(-1)$ be a non-totally geodesic, minimal immersion of a space-like surface $M^{2}$ into $H_{2}^{4}(-1)$. Then

$$
G+K^{D} \geq-1
$$


holds identically on $M^{2}$.

If $G+1$ is logarithm-harmonic, then the equality sign of (6.3) holds identically if and only if $\psi: M^{2} \rightarrow H_{2}^{4}(-1)$ is congruent to an open portion of the immersion $\psi_{\phi}: H^{2}\left(-\frac{1}{3}\right) \rightarrow H_{2}^{4}(-1)$ which is induced from the map $\phi: \mathbf{R}^{2} \rightarrow \mathbb{E}_{3}^{5}$ defined by

$$
\begin{gathered}
\phi(s, t)=\left(\sinh \left(\frac{2 s}{\sqrt{3}}\right)-\frac{t^{2}}{3}-\left(\frac{7}{8}+\frac{t^{4}}{18}\right) e^{\frac{2 s}{\sqrt{3}}}, t+\left(\frac{t^{3}}{3}-\frac{t}{4}\right) e^{\frac{2 s}{\sqrt{3}}}, \frac{1}{2}+\frac{t^{2}}{2} e^{\frac{2 s}{\sqrt{3}}}, t+\left(\frac{t^{3}}{3}+\frac{t}{4}\right) e^{\frac{2 s}{\sqrt{3}}},\right. \\
\left.\sinh \left(\frac{2 s}{\sqrt{3}}\right)-\frac{t^{2}}{3}-\left(\frac{1}{8}+\frac{t^{4}}{18}\right) e^{\frac{2 s}{\sqrt{3}}}\right) .
\end{gathered}
$$

Corollary 6.1. [38] Let $\psi: M^{2} \rightarrow H_{2}^{4}(-1)$ be a minimal immersion of a space-like surface $M^{2}$ of constant Gauss curvature into $H_{2}^{4}(-1)$. Then the equality sign of (6.3) holds identically if and only if one of the following two statements holds.

(1) $G=-1, K^{D}=0$, and $\psi$ is totally geodesic.

(2) $K^{D}=2 G=-\frac{2}{3}$ and $\psi$ is congruent to an open part of the minimal surface $\psi_{\phi}: H^{2}\left(-\frac{1}{3}\right) \rightarrow H_{2}^{4}(-1)$ induced from $\psi$ defined by (6.4).

Proposition 6.1. [38] Let $\psi: M^{2} \rightarrow \mathbb{E}_{2}^{4}$ be a minimal immersion of a space-like surface $M^{2}$ into the pseudo-Euclidean 4 -space $\mathbb{E}_{2}^{4}$. Then

$$
G \geq-K^{D}
$$

holds identically on $M^{2}$. Further, if $M^{2}$ has constant Gauss curvature, then the equality sign of (6.5) holds identically if and only if $M^{2}$ is a totally geodesic surface.

Proposition 6.2. [38] Let $\psi: M^{2} \rightarrow \mathbb{E}_{2}^{4}$ be a minimal immersion of a space-like surface $M^{2}$ into $\mathbb{E}_{2}^{4}$. Then

(1) If the equality sign of (6.3) holds identically, then $K$ is a non-logarithm-harmonic function.

(2) If $M^{2}$ contains no totally geodesic points and the equality sign of (6.5) holds identically on $M^{2}$, then $\ln G$ is a subharmonic function.

Proposition 6.3. [38] Let $\psi: M^{2} \rightarrow S_{2}^{4}(1)$ be a minimal immersion of a space-like surface $M^{2}$ into the neutral pseudosphere $S_{2}^{4}(1)$. Then

$$
G+K^{D} \geq 1
$$

holds identically on $M^{2}$. Further, if $M^{2}$ has constant Gauss curvature, then the equality sign of (6.6) holds identically if and only if $M^{2}$ is a totally geodesic surface.

Proposition 6.4. [38] Let $\psi: M^{2} \rightarrow S_{2}^{4}(1)$ be a minimal immersion of a space-like surface $M^{2}$ into $S_{2}^{4}(1)$. We have

(1) If the equality sign of (6.6) holds identically, then $G-1$ is non-logarithm-harmonic.

(2) If $M^{2}$ contains no totally geodesic points and if the equality case of (6.6) holds, then $\ln (G-1)$ is subharmonic.

\section{DDVV conjecture and theorem}

\section{1. $D D V V$ conjecture}

Suppose that $M^{n}$ is a submanifold of a real space form $R^{m}(c)$. Then the normalized scalar curvature $\rho$ of $M^{n}$ is defined by

$$
\rho=\frac{2}{n(n-1)} \sum_{i<j}\left\langle R\left(e_{i}, e_{j}\right) e_{j}, e_{i}\right\rangle,
$$

where $R$ is the Riemann curvature tensor of $M^{n}$ and $\left\{e_{1}, \ldots, e_{n}\right\}$ is an orthonormal frame of the tangent bundle $T M^{n}$. 
P. J. De Smet, F. Dillen, L. Vrancken and L. Verstraelen defined the normalized normal scalar curvature $\rho^{\perp}$ of $M^{n}$ in [61] as

$$
\rho^{\perp}=\frac{2}{n(n-1)} \sqrt{\sum_{1 \leq i<j \leq n ; 1 \leq r<s \leq m-n}\left\langle R^{\perp}\left(e_{i}, e_{j}\right) \xi_{r}, \xi_{s}\right\rangle^{2}},
$$

where $R^{\perp}$ is the normal connection of $M^{n}$, and $\left\{e_{1}, \ldots, e_{n}\right\}$ and $\left\{\xi_{1}, \ldots, \xi_{m-n}\right\}$ are orthonormal frames of the tangent and normal bundles of $M^{n}$, respectively.

In 1999, De Smet, Dillen, Vrancken and Verstraelen [61] formulated the following conjecture as the (generalized) Wintgen's inequality in general case which is well-known as the DDVV conjecture.

Conjecture 1. Let $\phi: M^{n} \rightarrow R^{m}(c)$ be an isometric immersion of an Riemannian n-manifold $M^{n}$ into a real space form $R^{m}(c)$ of constant curvature $c$. Then

$$
\rho \leq\|H\|^{2}-\rho^{\perp}+c
$$

holds at each point $p \in M^{n}$.

For normally flat submanifolds, i.e., $R^{D}=0$, the normal scalar curvature vanished. In this case, the inequality (7.3) was proved earlier in 1996 by the author in [27]. Hence, the DDVV conjecture holds true for hypersurfaces of real space form [27]. Further, this conjecture was also proved by De Smet et. al. for submanifolds with codimension 2 in real space forms $R^{n+2}(c)$ in [61].

\subsection{The Solution of DDVV conjecture by Z. Lu, J. Ge and Z. Tang}

The DDVV conjecture was finally settled for general case by Z. Lu [94] and J. Ge and Z. Tang [78] independently. Consequently, we have the following.

Theorem 7.1. The Wintgen inequality (7.3) holds for every submanifold $M^{n}, n \geq 2$, in any real space form $R^{m}(c)$ with $m \geq 4$.

The equality case of (7.3) holds at $p \in M^{n}$ if and only if there exists an orthonormal basis $\left\{e_{1}, \cdots, e_{n}\right\}$ of the tangent space $T_{p} M^{n}$ and an orthonormal basis $\left\{n_{1}, \cdots, n_{m-n}\right\}$ of the normal space $T_{p}^{\perp} M^{n}$, such that the shape operators $\left\{A_{r}, r=1, \cdots, m-n\right\}$ take the form as below:

$$
\begin{aligned}
A_{1}=\left(\begin{array}{ccccc}
\lambda_{1} & \mu_{0} & 0 & \cdots & 0 \\
\mu_{0} & \lambda_{1} & 0 & \cdots & 0 \\
0 & 0 & \lambda_{1} & \cdots & 0 \\
\vdots & \vdots & \vdots & \ddots & \vdots \\
0 & 0 & 0 & \cdots & \lambda_{1}
\end{array}\right), A_{2}=\left(\begin{array}{ccccc}
\lambda_{2}+\mu_{0} & 0 & 0 & \cdots & 0 \\
0 & \lambda_{2}-\mu_{0} & 0 & \cdots & 0 \\
0 & 0 & \lambda_{2} & \cdots & 0 \\
\vdots & \vdots & \vdots & \ddots & \vdots \\
0 & 0 & 0 & \cdots & \lambda_{2}
\end{array}\right), \\
A_{3}=\lambda_{3} I_{n}, A_{s}=0(s=4, \ldots, m-n),
\end{aligned}
$$

where $I_{n}$ is the identity matrix.

In the following, the inequality (7.3) is also called the Wintgen inequality and a submanifold $M^{n}$ of a real space form $M^{m}(c)$ is called a generalized Wintgen ideal submanifold (or simply a Wintgen ideal submanifold) if it satisfies the equality case of the Wintgen inequality (7.3) identically.

It follows from Theorem 7.1 that, for a non-totally umbilical Wintgen ideal submanifold $M^{n}$, there exists a special 2-dimensional distribution spanned by $e_{1}, e_{2}$ such that the shape operators of $M^{n}$ takes the special form (7.4). We denote this special 2-dimensional distribution by $\mathfrak{D}_{2}$ so that we have

$$
\mathfrak{D}_{2}=\operatorname{Span}\left\{e_{1}, e_{2}\right\} .
$$

\section{Some properties of Wintgen ideal submanifolds}

In this section, we present some general results for Wintgen ideal submanifolds in real space forms. 


\subsection{Wintgen ideal submanifolds of real space forms are Chen submanifolds}

The following general property of Wintgen ideal submanifold was proved by S. Decu, M. Petrović-Torgašev and L. Verstraelen in [70],

Theorem 8.1. Every Wintgen ideal submanifold of arbitrary dimension and arbitrary codimension in a real space form is a Chen submanifold.

\subsection{Ricci and Casorati principal directions of Wintgen ideal submanifolds}

For a submanifold $M^{n}$ in a real space form $\widetilde{M}^{m}(c)$ of constant sectional curvature $c$, the Casorati curvature $\mathcal{C}$ and the Casorati operator of $M^{n}$ are defined respectively by

$$
\mathcal{C}=\frac{1}{n} \sum_{r=n+1}^{m}\left(\sum_{i, j=1}^{n}\left(h_{i j}^{r}\right)^{2}\right), \quad A^{C}=\sum_{r=n+1}^{m} A_{r}^{2} .
$$

The eigenvectors and eigenvalues of Casorati operator $A^{C}$ are called the principal Casorati directions and principal Casorati curvatures, respectively, so that the principal Casorati curvatures $\mathcal{C}_{1}, \ldots, \mathcal{C}_{n}$ satisfy $\mathcal{C}=\mathcal{C}_{1}+\cdots+\mathcal{C}_{n}$.

By contraction, it follows from the equation (2.6) of Gauss that the Ricci tensor $S$ satisfies

$$
\operatorname{Ric}(Y, Z)=(n-1) c g(Y, Z)+n g\left(A_{\vec{H}}(Y), Z\right)-g\left(A^{C}(Y), Z\right) .
$$

In terms of Casorati operator, (8.2) can be simply expressed as

$$
S(X)=c(n-1) X+n A_{H} X-A^{C}(X),
$$

where $S$ is the Ricci operator defined by $\langle S(X), Y\rangle=\operatorname{Ric}(X, Y)$.

The following general property of Wintgen ideal submanifold was proved by S. Decu, M. Petrović-Torgašev, A. Šebeković and L. Verstraelen in [68].

Theorem 8.2. On every Wintgen ideal submanifold in a real space form the Casorati and the Ricci principal directions do coincide.

Remark 8.1. S. Decu, S. Haesen and L. Verstraelen [64] introduced in 2007 the notion of $\delta$-curvature invariants. The study of $\delta$-Casorati curvatures is another very active research subject during the last decade. For a comprehensive survey on results in $\delta$-Casorati curvatures, we refer to a very recent survey article [47].

\subsection{Some classification theorems for Wintgen ideal submanifolds}

The following three classification results for Wintgen ideal submanifolds of codimension two were obtained by De Smet, Dillen, Vrancken and Verstraelen in [61].

Theorem 8.3. Let $\phi: M^{n} \rightarrow R^{n+2}(c)$ be an isometric immersion realizing at every point the equality in (7.3). If $M^{n}$ has constant nonzero mean curvature, then $M^{n}$ is totally umbilical.

Theorem 8.4. Let $\phi: M^{n} \rightarrow R^{n+2}(c), n \geq 3$ be an isometric immersion realizing at every point $x \in M^{n}$ the equality in (7.3). If $\rho^{\perp}$ is a nonzero constant, then $\phi$ is minimal.

Theorem 8.5. Let $\phi: M^{n} \rightarrow R^{n+2}(c), n \geq 3$ and $c \in\{-1,0,1\}$, be an immersion realizing at every point the equality in (7.3). If $M^{n}$ has constant nonzero normal curvature, then $n=3, c=1$ and $\phi$ is (locally) congruent to the lift of the holomorphic curve of constant curvature 2 in a complex projective plane $C P^{2}(4)$ of constant holomorphic sectional curvature 4 with Hopf's fibrations (see [114] or [40]).

For Wintgen ideal submanifolds of codimension $\geq 3$, Z. Xie [139] obtained the following results.

Theorem 8.6. Let $\phi: M^{n} \rightarrow R^{m}(c)$ be a Wintgen ideal submanifold with $n \geq 3$ and $m \geq n+3$. If the mean curvature of $M^{n}$ is constant, then locally

(1) $\phi$ is a totally umbilical submanifold, or

(2) $\phi$ is a minimal Wintgen ideal submanifold of $R^{m}(c)$, or

(3) $\phi$ lies in an umbilical hypersurface of $R^{m}(c)$ as a minimal Wintgen ideal submanifold. 
Theorem 8.7. Suppose $\phi: M^{n} \rightarrow R^{m}(c)$ is a Wintgen ideal submanifold with $n \geq 3$ and $m \geq n+2$. If the normal scalar curvature $\rho^{\perp}$ is constant, then locally

(1) $\phi$ is a totally umbilical submanifold; or

(2) $\phi$ is Möbius equivalent to a cone, cylinder, or rotational submanifold generated by a super-minimal surface in low dimensional space form; or

(3) $n=3$ and $\phi$ is a minimal Wintgen ideal submanifold of dimension three in $R^{m}(c)$ with $c>0$; or

(4) $n=3$ and $\phi$ lies in an umbilical hypersurface $\tilde{R}^{m-1}(\tilde{c})$ of $R^{m}(c)$ as a minimal Wintgen ideal submanifold of dimension three with $\tilde{c}>0$.

Theorem 8.8. Let $\phi: M^{n} \rightarrow R^{m}(c)$ be a Wintgen ideal submanifold with $n \geq 3$ and $m \geq n+2$. If the scalar curvature $\rho$ is constant, then locally

(1) $\phi$ is a totally umbilical submanifold; or

(2) $\phi$ is Möbius equivalent to a cone, cylinder, or rotational submanifold generated by a super-minimal surface in low dimensional space form; or

(3) $n=3$ and $\phi$ is a minimal Wintgen ideal submanifold in $R^{m}(c)$ with $c>0$; or

(4) $n=3$ and $\phi$ lies in an umbilical hypersurface $\tilde{R}^{m-1}(\tilde{c})$ of $R^{m}(c)$ as a minimal Wintgen ideal submanifold with $\tilde{c}>0$.

Remark 8.2. For more results of Wintgen ideal submanifolds in Möbius geometry, see §14.

\section{Wintgen ideal submanifolds in Kaehler manifolds}

Let $\widetilde{M}^{m}(4 c)$ be a complex space form of constant holomorphic sectional curvature $4 c$. Then the Riemannian curvature tensor field $\widetilde{R}$ takes the following expression:

$$
\begin{aligned}
\widetilde{R}(X, Y, Z, W)= & c\{g(X, Z) g(Y, W)-g(X, W) g(Y, Z)+g(J X, Z) g(J Y, W) \\
& -g(J X, W) g(J Y, Z)+2 g(J X, Y) g(J Z, W)\},
\end{aligned}
$$

for vector fields $X, Y, Z, W$ tangent to $\widetilde{M}^{m}(4 c)$, where $J$ denotes the almost complex structure on $\widetilde{M}^{m}(4 c)$.

According to the behavior of the tangent spaces under the action of $J$, we distinguish three fundamental classes of submanifolds in a complex space form.

(a) A submanifold $M^{n}$ is said to be a complex submanifold if $J\left(T_{x} M^{n}\right)=T_{x} M^{n}$, at any point $x \in M^{n}$ (cf. [106]).

(b) A submanifold $M^{n}$ is called totally real if $J\left(T_{x} M^{n}\right) \subset T_{x}^{\perp} M^{n}$, at any point $x \in M^{n}$. In particular, a totally real submanifold of maximum dimension $(n=m)$ is called Lagrangian (see [40,51]).

(c) A submanifold $M^{n}$ of a complex space form $\widetilde{M}^{m}(4 c)$ is calledslant if for any point $x \in M^{n}$ and any nonzero vector $X \in T_{x} M^{n}$, the angle between $J X$ and $T_{x} M^{n}$ is a constant $\theta$ (see [23, 24]).

9.1. Normal scalar curvature for complex submanifolds

For a complex submanifold $M^{n}$ in a complex space form $\widetilde{M}^{m}(c)$, let $\tau$ and $\tau^{\perp}$ denote the (non-normalized) scalar curvature and (non-normalized) normal scalar curvature of $M^{n}$ so that

$$
\tau=\frac{n(n-2)}{2} \rho, \quad \tau^{\perp}=\frac{n(n-2)}{2} \rho^{\perp} .
$$

In [71], F. Dillen, J. Fastenakels, J. Van der Veken proved the following inequalities for complex submanifolds in the complex space forms.

Theorem 9.1. Let $M^{2 n}$ be an invariant submanifold of a complex space form $\widetilde{M}^{m}(c)$. Then we have

$[(\mathrm{i})] 4 n\left(\tau^{\perp}\right)^{2} \geq[n(n+2) c-2 \tau]^{2}+n^{2}(m-n-1) c^{2}$ holds, with equality holding identically if and only if $M^{2 n}$ is an Einstein manifold, and

[(ii) $] 4\left(\tau^{\perp}\right)^{2} \leq\left[\left(n^{2}+n+1\right) c-2 \tau\right]^{2}+\left(m n-n^{2}-1\right) c^{2}$ holds, with equality holding identically if and only if the complex rank of $\mathcal{A}=\sum_{\alpha=1}^{2 m} A_{\alpha}^{2}$ is at most 1 .

Theorem 9.1 implies the following.

Corollary 9.1. For an invariant submanifold $M^{n}$ of a complex Euclidean $m$-space $\mathbf{C}^{m}$, we have $\rho \leq-\rho^{\perp}$. 


\subsection{Wintgen inequality for totally and Lagrangian submanifolds}

I. Mihai shown in [103] that Theorem 7.1 holds true for totally real submanifolds in a complex space form of constant holomorphic sectional curvature $4 c$. On the other hand, for Lagrangian submanifolds in complex space forms, I. Mihai proved the following inequality.

Theorem 9.2. [101] Let $M^{n}$ be a Lagrangian submanifold of a complex space form $\widetilde{M}^{m}(4 c)$. Then we have

$$
\left(\rho^{\perp}\right)^{2} \leq\left(\|H\|^{2}-\rho+c\right)^{2}+\frac{4}{n(n-1)}(\rho-c) c+\frac{2 c^{2}}{n(n-1)} .
$$

The equality case holds identically if and only if, with respect to suitable orthonormal frames $\left\{e_{1}, \ldots, e_{n}\right\}$ and $\left\{\xi_{1}, \ldots, \xi_{m-n}\right\}$, the shape operators of $M^{n}$ in $\widetilde{M}^{m}(4 c)$ take the forms

$$
\begin{aligned}
A_{\xi_{1}} & =\left(\begin{array}{ccccc}
\lambda_{1} & \mu & 0 & \cdots & 0 \\
\mu & \lambda_{1} & 0 & \cdots & 0 \\
0 & 0 & \lambda_{1} & \cdots & 0 \\
\vdots & \vdots & \vdots & \ddots & \vdots \\
0 & 0 & 0 & \cdots & \lambda_{1}
\end{array}\right), A_{\xi_{2}}=\left(\begin{array}{ccccc}
\lambda_{2}+\mu & 0 & 0 & \cdots & 0 \\
0 & \lambda_{2}-\mu & 0 & \cdots & 0 \\
0 & 0 & \lambda_{2} & \cdots & 0 \\
\vdots & \vdots & \vdots & \ddots & \vdots \\
0 & 0 & 0 & \cdots & \lambda_{2}
\end{array}\right), \\
A_{\xi_{3}} & =\lambda_{3} I_{n}, \quad A_{\xi_{4}}=\cdots=A_{\xi_{2 m-n}}=0,
\end{aligned}
$$

where $\lambda_{1}, \lambda_{2}, \lambda_{3}$ and $\mu$ are real functions on $M^{n}$.

Theorem 9.2 implies the following.

Corollary 9.2. [101] Any Wintgen ideal Lagrangian submanifold of a complex space form is a Chen submanifold.

Remark 9.1. Let $f: M^{2} \rightarrow S^{5}$ be a minimal isometric Legendrian immersion of a surface $M^{2}$ in the 5dimensional sphere $S^{5}$ and define

$$
x:\left(0, \frac{\pi}{2}\right) \times \cos t M^{2} \times \sin t S^{n-3} \rightarrow S^{2 n+1},(t, p, q) \mapsto(\cos t) f(p)+(\sin t) q .
$$

Then its image under the Hopf fibration $\pi: S^{2 n+1} \rightarrow P^{n}(\mathbf{C})$ satisfies the equality case of (9.1) in Theorem 2.3 because their shape operators have the desired forms (see [48]).

Remark 9.2. We have:

(a) The inequality (9.1) in Theorem 9.2 with $n=3$ was established by A. Mihai [98].

(b) The inequality in Corollary 9.2 with $n=3,4$ was given by Dillen, Fastenakels and Van der Veken in [72].

9.3. Wintgen inequality for slant and CR-submanifolds

The following Wintgen type inequality for slant submanifolds was obtained by I. Mihai.

Theorem 9.3. [101] Let $M^{n}$ be an n-dimensional $\theta$-slant submanifold of a complex space form $\widetilde{M}^{m}(4 c)$. Then we have

$$
\|H\|^{2} \geq \rho+\rho^{\perp}-c-\frac{3 c}{n-1} \cos ^{2} \theta
$$

The next corollary from [101] follows immediately from Theorem 9.3.

Corollary 9.3. Let $M^{n}$ be an $n$-dimensional $\theta$-slant submanifold of $\mathbf{C}^{m}$. Then

$$
\|H\|^{2} \geq \rho+\rho^{\perp} .
$$

A submanifold $M^{n}$ of a Kaehler manifold $\left(\widetilde{M}^{m}, \tilde{g}, J\right)$ is called a $C R$-submanifold if there exist a holomorphic distribution $\mathcal{D}$ and a totally real distribution $\mathcal{D}^{\perp}$ on $M^{n}$ such that $T M^{n}=\mathcal{D} \oplus \mathcal{D}^{\perp}$, where $T M^{n}$ denotes the tangent bundle of $M^{n}$. The notion of $C R$-submanifolds was introduced by A. Bejancu (cf. [13]). [99].

For CR-submanifolds of a complex space form $\widetilde{M}^{m}(4 c)$, A. Mihai and I. Mihai proved the following result in

Theorem 9.4. Let $M^{n}$ be an $n$-dimensional $C R$-submanifold of a complex space form $\widetilde{M}^{m}(4 c)$. Then

$$
\|H\|^{2} \geq \rho+\rho^{\perp}-c-\frac{6 \alpha c}{n(n-1)},
$$

where $\alpha=\operatorname{rank}_{\mathbf{C}}(\mathcal{D})$. 


\subsection{Bi-slant submanifolds in locally conformal Kaehler space forms}

A locally conformally Kaehler manifold $(\widetilde{M}, J, g)$ (or simply an $L C K$-manifold) is a Hermitian manifold $(\widetilde{M}, J, g)$ which is locally conformal to a Kaehler manifold. Equivalently, there exists an open cover $\left\{U_{i}\right\}_{i \in I}$ of $\widetilde{M}$ and a family $\left\{f_{i}\right\}_{i \in I}$ of real-valued differentiable functions $f_{i}: U_{i} \rightarrow \mathbb{R}$ such that $g_{i}=\left.e^{-f_{i}} g\right|_{U_{i}}$ is a Kaehlerian metric on $U_{i}$, i.e., $\nabla^{*} J=0$, where $J$ is the almost complex structure, $g$ is the Hermitian metric, and $\nabla^{*}$ is the covariant differentiation with respect to $g$ (see, e.g., [73]).

A typical example of a compact $L C K$-manifold is a Hopf manifold which is diffeomorphic to $\mathbb{S}^{1} \times \mathbb{S}^{2 n-1}$ and it admits no Kaehler structure (see [132]).

A locally conformal Kaehler manifold with constant holomorphic sectional curvature $c$ is called a locally conformal Kaehler space form and it is denoted by $\widetilde{\mathcal{N}}(c)$. The curvature tensor $\widetilde{R}$ of a locally conformal Kaehler space form of constant holomorphic sectional curvature $c$ is given (see, e.g., $[88,97]$ )

$$
\begin{aligned}
\widetilde{R}(X, Y ; Z, W)= & \frac{c}{4}\{g(X, W) g(Y, Z)-g(X, Z) g(Y, W)\} \\
& +\frac{c}{4}\{g(J X, W) g(J Y, Z)-g(J X, Z) g(J Y, W)-2 g(J X, Y) g(J Z, W)\} \\
& -\frac{3}{4}\{g(Y, Z) \bar{P}(X, W)-g(X, Z) \bar{P}(Y, W)+g(X, W) \bar{P}(Y, Z)-g(Y, W) \bar{P}(X, Z)\},
\end{aligned}
$$

where $\bar{P}$ is a hybrid tensor field on $\widetilde{\mathcal{N}}(c)$, i.e., a $(0,2)$-tensor field satisfies

$$
\bar{P}(J X, Y)+\bar{P}(X, J Y)=0
$$

for any vector fields $X, Y$ tangent to $\widetilde{M}$.

Remark 9.3. Compact locally conformal Kaehler space forms of zero curvature were classified by I. Vaisman in [132] (see also [73, Theorem 6.8]).

A submanifold $M^{n}$ of an almost Hermitian manifold $\widetilde{M}^{m}$ is called bi-slant if there exist two orthogonal distributions $\mathcal{D}_{1}$ and $\mathcal{D}_{2}$ such that the following three conditions are satisfied (see [21]):

(i) $T M^{n}$ admits the orthogonal direct decomposition i.e., $T M^{n}=\mathcal{D}_{1} \oplus \mathcal{D}_{2}$.

(ii) $J \mathcal{D}_{1} \perp \mathcal{D}_{2}$ and $J \mathcal{D}_{2} \perp \mathcal{D}_{1}$

(iii) $\mathcal{D}_{1}$ and $\mathcal{D}_{2}$ are the slant distribution with slant angle $\theta_{1}$ and $\theta_{2}$, respectively.

Remark 9.4. A bi-slant submanifold is a CR-submanifold (resp., semi-slant; hemi-slant; or slant submanifold) if we have $\theta_{1}=0, \theta_{2}=\frac{\pi}{2}$ (resp., $\theta_{1}=0,0<\theta_{2}<\frac{\pi}{2} ; \theta_{1}=\frac{\pi}{2}, 0<\theta_{2}<\frac{\pi}{2}$; or $\mathcal{D}_{1}=\{0\}$ and $\mathcal{D}_{2} \neq\{0\}$ ).

For bi-slant submanifolds in locally conformal Kaehler space forms, M. Aquib, M. S. Lone and M. A. Lone obtained the following result in [5].

Theorem 9.5. Let $M^{n}$ be an n-dimensional bi-slant submanifold of a locally conformal Kaehler space form $\widetilde{N}^{m}(c)$. Then

$$
\begin{aligned}
\rho^{\perp} \leq & \|H\|^{2}-(\rho-c)-\frac{3}{n-1} \operatorname{trace}(\bar{P})+\frac{c}{2 n(n-1)}\left(d_{1} \cos ^{2} \theta_{1}+d_{1} \cos ^{2} \theta_{2}\right) \\
& -\frac{3}{2 n(n-1)} \sum_{1 \leq i<j \leq n} g\left(J e_{i}, e_{j}\right) \bar{P}\left(J e_{i}, e_{j}\right),
\end{aligned}
$$

where $d_{i}=\frac{1}{2} \operatorname{rank}\left(\mathcal{D}_{i}\right)(i=1,2)$ and $\left\{e_{1}, \ldots, e_{n}\right\}$ is an orthonormal frame of $T M^{n}$.

\section{Wintgen ideal submanifolds in Sasakian space forms}

\subsection{Almost contact metric manifold and Sasakian space forms}

A Riemannian $(2 n+1)$-manifold $\left(\bar{M}^{2 n+1}, g\right)$ is called an almost contact metric manifold (see [14]) if there exist a $(1,1)$ tensor field $\varphi$, a vector field $\xi$ (called the structure vector field), and a 1-form $\eta$ on $\bar{M}^{2 n+1}$ such that

$$
\begin{aligned}
& \eta(\xi)=1, \quad \varphi^{2}(X)=-X+\eta(X) \xi, \quad \varphi \xi=0, \quad \eta \circ \varphi=0, \\
& g(\varphi X, \varphi Y)=g(X, Y)-\eta(X) \eta(Y), \quad \eta(X)=g(X, \xi)
\end{aligned}
$$


for vector fields $X, Y$ tangent to $\bar{M}^{2 n+1}$. An almost contact metric manifold $\left(\bar{M}^{2 n+1}, \varphi, \xi, \eta, g\right)$ is called a Sasakian manifold if it satisfies

$$
\left(\nabla_{X} \varphi\right) Y=g(X, Y) \xi-\eta(Y) X .
$$

A Sasakian manifold is called Sasakian space form if it has constant $\varphi$-sectional curvature. The curvature tensor $\bar{R}$ of a Sasakian space form of constant $\bar{M}^{2 n+1}(c)$ constant $\varphi$-sectional curvature $c$ is given by

$$
\begin{aligned}
\bar{R}(X, Y) Z= & \frac{c+3}{4}\{g(Y, Z) X-g(X, Z) Y\}+\frac{c-1}{4}\{g(X, \varphi Z) \varphi Y-g(Y, \varphi Z) \varphi X+2 g(X, \varphi Y) \varphi Z\} \\
& +\frac{c-1}{4}\{\eta(X) \eta(Z) Y-\eta(Y) \eta(Z) X+g(X, Z) \eta(Y) \xi-g(Y, Z) \eta(X) \xi\}
\end{aligned}
$$

for $X, Y, Z$ tangent to $\bar{M}^{2 n+1}$.

Sasakian space forms $\bar{M}^{2 n+1}(c)$ can be modeled based on $c>-3, c=-3$ or $c<-3$. For instance, $\mathbb{R}^{2 m+1}$ has constant $\varphi$-sectional curvature -3 , while $S^{2 m+1}(1)$ is of constant $\varphi$-sectional curvature one.

\subsection{Invariant submanifolds in Sasakian space forms}

A $(2 n+1)$-dimensional submanifold $M^{2 n+1}$ of a $(2 m+1)$-dimensional Sasakian manifold $\left(\bar{M}^{2 n+1}, \varphi, \xi, \eta, g\right)$ is called invariant if the structure vector field $\xi$ is tangent to $M^{2 n+1}$ and $\varphi\left(T_{x} M^{2 n+1}\right) \subset T_{x} M^{2 n+1}$ for any $x \in M^{2 n+1}$. It is well-known that every invariant submanifold of a Sasakian manifold with the induced structure tensors is again a Sasakian manifold.

Analogous to Theorem 9.1, F. Dillen, J. Fastenakels and J. Van der Veken proved the following inequalities for invariant submanifolds in Sasakian space forms.

Theorem 10.1. [71] Let $M^{2 n+1}$ be an invariant submanifold of a Sasakian space form $\bar{M}^{2 m+1}$ (c) of constant $\varphi$ holomorphic sectional curvature $c$. Then

(i) $4 n\left(\tau^{\perp}\right)^{2} \geq\left(n(n+2) c+3 n^{2}-2 \tau\right)^{2}+n^{2}(m-n-1)(c-1)^{2}$ holds, with equality holding identically if and only if $M^{2 n}$ is $\eta$-Einstein, and

(ii) $4\left(\tau^{\perp}\right)^{2} \leq\left[\left(n^{2}+n+1\right) c+\left(3 n^{2}+n-1\right)-2 \tau\right]^{2}+\left(m n-n^{2}-1\right)(c-1)^{2}$ holds, with equality holding identically if and only if the complex rank of $\mathcal{A}=\sum_{\alpha=1}^{2 m} A_{\alpha}^{2}$ is at most 2 .

Theorem 10.1 implies the following.

Corollary 10.1. For an invariant submanifold $M^{2 n+1}$ of $S^{2 m+1}(1)$, we have $\rho \leq 1-\rho^{\perp}$.

10.3. C-totally real, Legendrian and slant submanifolds in Sasakian space forms

An $n$-dimensional submanifold $M^{n}$ of a almost contact metric manifold $\left(\widetilde{M}^{2 m+1}, \varphi, \xi, \eta, g\right)$ is called $C$ totally real if the structure vector field $\xi$ is normal to $M^{n}$. It follows that $\varphi\left(T_{x} M^{n}\right) \subset T_{x}^{\perp} M^{n}$ for $C$-totally real submanifolds. A $C$-totally real submanifold is called Legendrian if $n=m$. Thus, a Legendrian submanifold is a $C$-totally real submanifold with the smallest possible codimension.

For $C$-totally real submanifolds, I. Mihai [104] proved the following.

Proposition 10.1. Let $M^{n}$ be an $n$-dimensional C-totally real submanifold of a $(2 m+1)$-dimensional Sasakian space form $\bar{M}^{2 m+1}(c)$. Then we have

$$
\|H\|^{2}+\frac{c+3}{4} \geq \rho+\rho_{N} .
$$

The equality case of (10.1) holds identically if and only if, with respect to suitable orthonormal frames $\left\{e_{1}, \ldots, e_{n}\right\}$ and $\left\{\xi_{1}, \ldots, \xi_{2 m-n+1}\right\}$ with $\xi_{2 m-n+1}=\xi$, the shape operators of $M^{n}$ in $\bar{M}^{2 m+1}$ (c) take the forms of (7.4).

For Legendrian submanifolds, Mihai [104] proved the following Wintgen type inequality.

Theorem 10.2. Let $M^{n}$ be a Legendrian submanifold of a Sasakian space form $\bar{M}^{2 n+1}(c)$. Then

$$
\left(\rho^{\perp}\right)^{2} \leq\left(\|H\|^{2}-\rho+\frac{c+3}{4}\right)^{2}+\frac{4}{n(n-1)}\left(\rho-\frac{c+3}{4}\right) \cdot \frac{c-1}{4}+\frac{(c-1)^{2}}{8 n(n-1)} .
$$


The following are some easy consequences of Proposition 10.1 and Theorem 10.2.

Corollary 10.2. Let $M^{n}$ be a minimal Legendrian submanifold of $S^{2 n+1}(1)$. Then $\rho \leq 1-\rho^{\perp}$.

Corollary 10.3. Any Wintgen ideal Legendrian submanifold of a Sasakian space form is a Chen submanifold.

Recall that a submanifold $M^{n}$ tangent to $\xi$ of a Sasakian space form $\bar{M}^{2 m+1}(c)$ is said to be a contact slant submanifold if for any $p \in M^{n}$ and $X \in T_{p} M^{n}$ linearly independent on $\xi_{p}$, the angle between $\varphi X$ and $T_{p} M^{n}$ is a constant $\theta$, called the slant angle of $M^{n}$.

For slant submanifolds, Mihai [104] proved the following.

Theorem 10.3. Let $M^{n}$ be an $n$-dimensional contact $\theta$-slant submanifold of a $(2 m+1)$-dimensional Sasakian space form $\bar{M}^{2 m+1}(c)$. Then

$$
\rho+\rho_{N} \leq\|H\|^{2}+\frac{c+3}{4}+\frac{\left(3 \cos ^{2} \theta-2\right)(c-1)}{4 n} .
$$

In particular, Theorem 10.3 implies the following.

Corollary 10.4. Let $M^{n}$ be an $n$-dimensional contact slant submanifold of $S^{2 m+1}(1)$. Then $\rho+\rho^{\perp} \leq\|H\|^{2}+1$.

Remark 10.1. A Lorentzian manifold $M^{n}$ together with a unit time-like concircular vector field $\xi$, its associated 1-form $\eta$ and a (1,1)-tensor field $\varphi$ is called a Lorentzian concircular structure manifold (or simply a LCS-manifold). A. N. Siddiqui and K. Ahmad derived in [124] a Wintgen type inequality for totally real submanifolds and C-totally real submanifolds in LCS-manifolds with respect to the Levi-Civita connection as well as quarter symmetric metric connection.

\subsection{C-totally real, Legendrian and bi-slant submanifolds in Kenmotsu space forms}

An almost contact metric manifold $\left(\bar{M}^{2 m+1}, \varphi, \xi, \eta, g\right)$ is called a Kenmotsu manifold if it satisfies

$$
\left(\nabla_{X} \varphi\right) Y=g(\varphi X, Y) \xi-\eta(Y) \varphi X
$$

where $\nabla$ is the Levi-Civita connection of $\bar{M}^{2 m+1}$. Any Kenmotsu manifold can be obtained locally as follows (see [77, Proposition 3.2]): Let $\left(M_{0}, g_{0}, J\right)$ be an almost Hermitian manifold. Put $\bar{M}=M_{0} \times \mathbb{R}, \bar{g}=e^{2 t} g_{0}+$ $d t^{2}, \bar{\xi}=\frac{\partial}{\partial t}$ and define $\varphi$ by $\varphi X=J X$ for $X$ tangent to $\bar{M}$ and $\varphi \bar{\xi}=0$. Then we have

(1) The triple $(\bar{g}, \varphi, \bar{\xi})$ is an almost contact metric structure on $\bar{M}$.

(2) $\left(M_{0}, g_{0}, J\right)$ is a Kaehler manifold if and only if $(\bar{g}, \varphi, \bar{\xi})$ is a Kenmotsu structure on $\bar{M}$.

For $C$-totally real submanifolds in Kenmotsu space forms, M. Aquib and M. H. Shahid proved the following results in [6].

Proposition 10.2. Let $M^{n}$ be an $n$-dimensional $C$-totally real submanifold of a $(2 m+1)$-dimensional Kenmotsu space form $\widetilde{M}^{2 m+1}(c)$. Then we have

$$
\rho+\rho_{N} \leq\|H\|^{2}+\frac{c-3}{4}+\frac{c+1}{2 n} .
$$

The equality case holds identically if and only if, with respect to suitable orthonormal frames $\left\{e_{1}, \ldots, e_{n}\right\}$ and $\left\{e_{n+1}, \ldots, e_{2 m}, e_{2 m+1}=\xi\right\}$, the shape operators of $M^{n}$ in $\widetilde{M}^{2 m+1}(c)$ take the forms of (7.4).

For Legendrian submanifolds, Aquib and Shahid [6] proved the following.

Theorem 10.4. Let $M^{n}$ be a Legendrian submanifold of a Kenmotsu space form $\widetilde{M}^{2 n+1}(c)$. Then

$$
\begin{aligned}
\left(\rho^{\perp}\right)^{2} \leq & \left\{\|H\|^{2}-\left(\rho-\frac{c-3}{4}\right)+\frac{c+1}{2 n}\right\}^{2}+\frac{2}{n(n-1)}\left(\frac{c+1}{4}\right)^{2} \\
& +\frac{c+1}{n^{2}(n-1)^{2}}\left\{\left(n(n-1)\left(\rho-\frac{c-3}{4}\right)+\frac{c+1}{2}(n-1)\right\} .\right.
\end{aligned}
$$

A submanifold $M^{n}$ of an almost contact metric manifold is said to be a bi-slant submanifold, if there exist two orthogonal distributions $\mathcal{D}_{1}$ and $\mathcal{D}_{2}$, such that (1) $T M^{n}$ admits the orthogonal direct decomposition $T M^{n}=\mathcal{D}_{1} \oplus \mathcal{D}_{2} \oplus \operatorname{Span}\{\xi\}$ and (2) $\mathcal{D}_{1}$ and $\mathcal{D}_{2}$ are slant distributions with slant angle $\theta_{1}$ and $\theta_{2}$, respectively. We put $d_{i}=\frac{1}{2} \operatorname{rank}\left(\mathcal{D}_{i}\right), i=1,2$.

For bi-slant submanifolds, Aquib and Shahid [6] proved the following. 
Theorem 10.5. Let $M^{n}$ be a bi-slant submanifold of a Kenmotsu space form $\widetilde{M}^{2 n+1}(c)$. Then

$$
\left(\rho^{\perp}\right)^{2} \leq\|H\|^{2}-\left(\rho-\frac{c-3}{4}\right)-\frac{c+1}{2 n}+\frac{3(c+1)}{2 n(n-1)}\left(d_{1} \cos \theta_{1}+d_{2} \cos \theta_{2}\right) .
$$

In particular, Theorem 10.5 implies the following.

Corollary 10.5. Let $M^{n}$ be an invariant submanifold of of a Kenmotsu space form $\widetilde{M}^{2 n+1}(c)$. Then

$$
\rho^{\perp} \leq\|H\|^{2}-\left(\rho-\frac{c-3}{4}\right)-\frac{c+1}{2 n}+\frac{3(c+1)}{4(n-1)} .
$$

Corollary 10.6. Let $M^{n}$ be an anti-invariant submanifold of of a Kenmotsu space form $\widetilde{M}^{2 n+1}(c)$. Then

$$
\rho^{\perp} \leq\|H\|^{2}-\left(\rho-\frac{c-3}{4}\right)-\frac{c+1}{2 n} .
$$

Corollary 10.7. Let $M^{n}$ be a $\theta$-slant submanifold of of a Kenmotsu space form $\widetilde{M}^{2 n+1}(c)$. Then

$$
\rho^{\perp} \leq\|H\|^{2}-\left(\rho-\frac{c-3}{4}\right)-\frac{c+1}{2 n}+\frac{3(c+1)}{4 n(n-1)} \cos \theta .
$$

Corollary 10.8. Let $M^{n}$ be a CR-submanifold of of a Kenmotsu space form $\widetilde{M}^{2 n+1}(c)$. Then

$$
\rho^{\perp} \leq\|H\|^{2}-\left(\rho-\frac{c-3}{4}\right)-\frac{c+1}{2 n}+\frac{3(c+1)}{2 n(n-1)} d_{1} .
$$

\subsection{Legendrian submanifolds in generalized Sasakian-space-forms}

The notion of a generalized Sasakian space form was introduced by P. Alegre, D. E. Blair and A. Carriazo in [1] as follows. A $(2 m+1)$-dimensional manifold $\bar{M}^{2 m+1}$ equipped with an almost contact metric structure $(\varphi, \xi, \eta, g)$ is called a generalized Sasakian space form if there exist three functions $f_{1}, f_{2}, f_{3}$ on $\bar{M}^{2 m+1}$ such that the Riemann curvature tensor $\bar{R}$ of $\bar{M}^{2 m+1}$ satisfies

$$
\begin{aligned}
\bar{R}(X, Y) Z= & f_{1}\{g(Y, Z) X-g(X, Z) Y\}+f_{2}\{g(X, \varphi Z) \varphi Y-g(Y, \varphi Z) \varphi X+2 g(X, \varphi Y) \varphi Z\} \\
& +f_{3}\{\eta(X) \eta(Z) Y-\eta(Y) \eta(Z) X+g(X, Z) \eta(Y) \xi-g(Y, Z) \eta(X) \xi\} .
\end{aligned}
$$

We denote such a manifold by $\bar{M}^{2 m n+1}\left(f_{1}, f_{2}, f_{3}\right)$. The generalized Sasakian space form $\bar{M}^{2 m+1}\left(f_{1}, f_{2}, f_{3}\right)$ is a Sasakian space form if $f_{1}=\frac{c+3}{4}$ and $f_{2}=f_{3}=\frac{c-1}{4}$, where $c$ is a constant. Note that a Kenmotsu space form is a generalized Sasakian space form with $f_{1}=\frac{c-3}{4}$ and $f_{2}=f_{3}=\frac{c+1}{4}$.

Besides the Levi-Civita connection, S. Golasb introduced the notion of quarter symmetric connections in [81]. The Schouten-van Kampen connection was introduced in [118] for the study of non-holomorphic manifolds. The Tanaka-Webster connection is the canonical affine connection defined on a non-degenerate pseudoHermitian CR-manifold (see [129]). S. Tanno defined in [130] the Tanaka-Webster connection on contact metric manifolds.

In [86], S. K. Hui, R. S. Lemence and P. Mandal proved some Wintgen type inequalities for $C$-totally real and Legendrian submanifolds of $\bar{M}^{2 m+1}\left(f_{1}, f_{2}, f_{3}\right)$ with respect to quarter symmetric metric connection, the Schouten-van Kampen connection, and the Tanaka-Webster connection, respectively.

\section{Wintgen ideal submanifolds in quaternionic space forms}

\subsection{Quaternionic Kaehler manifold and quaternionic space forms}

A quaternionic Kaehler manifold is a Riemannian $4 m$-manifold whose Riemannian holonomy group is a subgroup of $S p(m) \cdot S p(1)$. An almost quaternionic Hermitian manifold $(\widehat{M}, \hat{g}, \Sigma)$ is a Riemannian manifold equipped with a rank 3-subbundle $\Sigma$ of $\operatorname{End}(T \widehat{M})$ with local basis $\left\{J_{1}, J_{2}, J_{3}\right\}$ satisfying

$$
\hat{g}\left(J_{\alpha} X, J_{\alpha} Y\right)=\hat{g}(X, Y), \quad J_{\alpha}^{2}=-I, \quad J_{\alpha} J_{\alpha+1}=-J_{\alpha+1} J_{\alpha}=J_{\alpha+2}, \quad X, Y \in T \widehat{M}
$$


for all $\alpha \in\{1,2,3\}$, where $I$ is the identity transformation on $T \widehat{M}$ and the indices are taken from $\{1,2,3\}$ modulo 3. Moreover, if the bundle $\Sigma$ is parallel with respect to the Levi-Civita connection of $\hat{g}$, then $(\widehat{M}, \hat{g}, \Sigma)$ is said to be a quaternionic Kaehler manifold (see [87]).

Let $X$ be a nonzero vector tangent to a quaternionic Kaehler manifold $(\widehat{M}, \hat{g} . \Sigma)$. Then the 4-plane $Q(X)$ spanned by $\left\{X, J_{1} X, J_{2} X, J_{3} X\right\}$, is called a quaternionic 4-plane. Any 2-plane in $Q(X)$ is called a quaternionic plane. The sectional curvature of a quaternionic plane is called a quaternionic sectional curvature. A quaternionic Kaehler manifold is called a quaternionic space form if its quaternionic sectional curvatures are equal to a constant. We denote by $\widehat{M}(c)$ a quaternionic space form of constant quaternionic sectional curvature $c$.

It is well known that the curvature tensor $\widehat{R}$ of a quaternionic space form $\widehat{M}(c)$ of constant quaternionic sectional curvature $c$ satisfies

$$
\widehat{R}(X, Y) Z=\frac{c}{4}\left\{\hat{g}(Z, Y) X-\hat{g}(X, Z) Y+\sum_{\alpha=1}^{3}\left[\hat{g}\left(Z, J_{\alpha} Y\right) J_{\alpha} X-\hat{g}\left(Z, J_{\alpha} X\right) J_{\alpha} Y+2 \hat{g}\left(X, J_{\alpha} Y\right) J_{\alpha} Z\right]\right\} .
$$

Let $M^{n}$ be a submanifold of a quaternionic Kaehler manifold $\widehat{M}^{4 m}$ of real dimension $4 m$. For any vector $X \in T_{x} M^{n}$, we put

$$
J_{\alpha} X=P_{\alpha} X+F_{\alpha} X, \quad P_{\alpha} X \in T_{x} M, \quad F_{\alpha} X \in T_{x}^{\perp} M^{n} .
$$

The squared norm of $P_{\alpha}$ is

$$
\left\|P_{\alpha}\right\|^{2}=\sum_{i, j=1}^{n} \hat{g}\left(P_{\alpha} e_{i}, e_{j}\right)^{2}
$$

where $\left\{e_{1}, \ldots, e_{n}\right\}$ is an orthonormal basis of $T_{x} M^{n}$.

\subsection{Lagrangian and slant submanifolds in quaternionic space forms}

A submanifold $M^{n}$ of a quaternionic Kaehler manifold $\widehat{M}^{4 m}$ of real dimension $4 m$ is called a $\theta$-slant or simply a slant submanifold if for each nonzero vector $X \in T_{x} M^{n}$, the angle $\theta(X)$ between $J_{\alpha}(X)$ and $T_{x} M^{n}, \alpha \in\{1,2,3\}$, is a global constant, so that it is independent of the choice of $p \in M^{n}$ and of $X \in T_{x} M^{n}$. A slant submanifold of a quaternionic Kaehler manifold is called proper (or proper $\theta$-slant) if $0<\theta<\frac{\pi}{2}$. A $\theta$-slant submanifold of a quaternionic Kaehler manifold is called totally real if $\theta=\frac{\pi}{2}$. And an $n$-dimensional totally real submanifold $M^{n}$ of a quaternionic Kaehler manifold $\widehat{M}^{4 m}$ is said to be a Lagrangian submanifold if $n=m$.

For Lagrangian submanifolds of a quaternionic space form $\widehat{M}^{4 m}(c)$, G. Macsim and V. Ghisoiu proved the following inequality in [96].

Theorem 11.1. Let $M^{n}$ be a Lagrangian submanifold of a quaternionic space form $\widehat{M}^{4 m}(c)$ of constant quaternionic sectional curvature $c$. Then

$$
\left(\rho^{\perp}\right)^{2} \leq\left(\|H\|^{2}-\rho+c\right)^{2}+\frac{6}{n(n-1)} c^{2}+\frac{4}{n(n-1)} c(\rho-c) .
$$

For slant submanifolds of $\bar{M}^{4 m}(c)$, Macsim and Ghisoiu [96] obtained the following.

Theorem 11.2. Let $M^{n}$ be a $\theta$-slant submanifold of a quaternionic space form $\widehat{M}^{4 m}(c)$. Then

$$
\|H\|^{2} \geq \rho+\rho^{\perp}-c-\frac{9 c}{n-1} \cos ^{\theta} .
$$

\subsection{Wintgen ideal inequality for quaternionic $C R$-submanifolds}

M. Barros, B.-Y. Chen and F. Urbano defined the notion of quaternionic CR-submanifolds of quaternionic Kaehler manifolds in [12] as a generalization of both quaternionic and totally real submanifolds. According to [12], a submanifold $M^{n}$ of a quaternionic Kaehler manifold $(\widehat{M}, \hat{g}, \Sigma)$ is said to be a quaternionic $C R$-submanifold if there exists two orthogonal complementary distributions $\mathcal{D}$ and $\mathcal{D}^{\perp}$ on $M^{n}$ such that $\mathcal{D}$ is invariant under quaternionic structure and $\mathcal{D}^{\perp}$ is totally real. It is obvious that a quaternionic CR-submanifold of a quaternionic Kaehler manifold reduces to a quaternionic submanifold (resp., to a totally real submanifold) if $\operatorname{dim} \mathcal{D}_{x}^{\perp}=0$, $x \in M^{n}$ (resp., $\left.\operatorname{dim} \mathcal{D}_{x}=0, x \in M\right)$. 
A quaternionic CR-submanifold $M^{n}$ is called proper if it is neither a quaternionic submanifold nor a totally real submanifold. In general, if $M^{n}$ is a quaternionic CR-submanifold of a quaternionic Kaehler manifold $(\widehat{M}, \hat{g}, \Sigma)$, then it is clear that the real dimension of $\mathcal{D}_{x}$ is divisible by 4 . Let us put $\operatorname{rank}_{\mathbb{H}} \mathcal{D}=p$ and $\operatorname{rank}_{\mathbb{R}} \mathcal{D}^{\perp}=q$.

For quaternionic CR-submanifolds, H. Alodan, B.-Y. Chen, S. Deshmukh, G.-E. Vilcu proved the following Wintgen's type inequality in [2].

Theorem 11.3. Let $M^{n}$ be a quaternionic $C R$-submanifold of a quaternionic space form $\widehat{M}^{4 m}(c)$ of constant quaternionic sectional curvature $c$. Then

$$
\rho \leq\|H\|^{2}-\rho^{\perp}+\frac{c}{4}+\frac{9 p c}{n(n-1)},
$$

where $p=\operatorname{rank}_{\mathbb{H}}(\mathcal{D})$.

Moreover, the equality sign holds in the above inequality at some point $x \in M^{n}$ if and only if there exists an orthonormal basis $\left\{e_{1}, \ldots, e_{n}\right\}$ of $T_{x} M^{n}$ and an orthonormal basis $\left\{\xi_{1}, \ldots, \xi_{4 m-n}\right\}$ of $T_{x}^{\perp} M^{n}$ such that the shape operators $A_{r} \equiv A_{\xi_{r}}$, $r=1, \ldots, 4 m-n$, take the forms of (7.4).

The following three corollaries are immediate consequence of Theorem 11.3.

Corollary 11.1. Let $M^{n}$ be a quaternionic CR-submanifold of the quaternionic Euclidean space $\mathbb{H}^{m}$. Then

$$
\|H\|^{2} \geq \rho+\rho^{\perp} .
$$

Corollary 11.2. Let $M^{n}$ be a quaternionic CR-submanifold of the quaternionic projective space $\mathbb{H} P^{m}(4)$ of constant quaternionic sectional curvature 4. Then

$$
\|H\|^{2} \geq \rho+\rho^{\perp}-1-\frac{36 p}{n(n-1)}
$$

where $p=\operatorname{rank}_{\mathbb{H}} \mathcal{D}$.

Corollary 11.3. Let $M^{n}$ be a quaternionic CR-submanifold of the quaternionic hyperbolic space $\mathbb{H} H^{m}(-4)$ of constant quaternionic sectional curvature -4 . Then

$$
\|H\|^{2} \geq \rho+\rho^{\perp}+1+\frac{36 p}{n(n-1)},
$$

where $p=\operatorname{rank}_{\mathbb{H}} \mathcal{D}$.

For quaternionic CR-submanifolds of a quaternionic space form with minimal codimension, the following result was also obtained in [2].

Theorem 11.4. Let $M^{n}$ be a quaternionic CR-submanifold of a quaternionic space form $\widehat{M}^{4 m}(c)$ with minimal codimension. If the dimension of $M^{n}$ is $n=4 p+q$, where $p=\operatorname{rank}_{\mathbb{H}} \mathcal{D}$ and $q=\operatorname{rank}_{\mathbb{R}} \mathcal{D}^{\perp}$, then the following inequality holds true:

$$
\left(\rho^{\perp}\right)^{2} \leq\left[\|H\|^{2}-\rho+\frac{c}{4}+\frac{9 p c}{n(n-1)}\right]^{2}+\frac{3 q(q-1) c^{2}}{8 n^{2}(n-1)^{2}}+\frac{c q(q-1)}{n^{2}(n-1)^{2}}\left(\rho_{\mathcal{D}^{\perp}}-\frac{c}{4}\right),
$$

where $\rho_{\mathcal{D}^{\perp}}$ denotes the normalized scalar curvature of the totally real distribution $\mathcal{D}^{\perp}$.

Moreover, the equality sign holds in the above inequality at some point $x \in M^{n}$ if and only if there exists an orthonormal basis $\left\{e_{1}, \ldots, e_{n}\right\}$ of $T_{x} M^{n}$ and an orthonormal basis $\left\{\xi_{1}, \ldots, \xi_{3 n}\right\}$ of $T_{x}^{\perp} M^{n}$ such that the shape operators $A_{r} \equiv A_{\xi_{r}}$, $r=1, \ldots, 3 q$, take the forms of (7.4).

Theorem 11.4 implies immediately the following.

Corollary 11.4. Let $M^{n}$ be a quaternionic CR-submanifold of the quaternionic Euclidean space $\mathbb{H}^{m}$. Then

$$
\rho^{\perp} \geq\left|\|H\|^{2}-\rho\right| \text {. }
$$

\section{Wintgen ideal submanifolds in golden Riemannian space forms}

A tensor field $F$ of type $(1,1)$ on a Riemannian manifold $(\widetilde{M}, \tilde{g})$ is called an almost product structure if it satisfies $F^{2}=I$. A Riemannian manifold $(\widetilde{M}, \tilde{g})$ endowed with an almost product structure $F$ is called an almost product Riemannian manifold if it satisfies $\tilde{g}(F X, Y)=\tilde{g}(X, F Y)$. 


\subsection{Locally Golden product space forms}

Let $(\widetilde{M}, \tilde{g})$ be a Riemannian $m$-manifold. A $(1,1)$-tensor field $\phi$ on $\widetilde{M}$ is called a golden structure if it satisfies

$$
\phi^{2}-\phi-I=0 .
$$

If the metric $\tilde{g}$ and a golden structure $\phi$ on $\widetilde{M}$ are compatible, i.e.,

$$
\widetilde{g}(\phi X, Y)=\tilde{g}(X, \phi Y)
$$

for any $X, Y \in T \widetilde{M}$, then $(\widetilde{M}, \tilde{g}, \phi)$ is called a golden Riemannian manifold [58]. The real positive root $\psi$ of the equation $x^{2}-x-1=0$, i.e., $\psi=\frac{1+\sqrt{5}}{2}$, is called the golden proportion.

Let $M^{n}$ be a submanifold of a golden Riemannian manifold $(\widetilde{M}, \tilde{g}, \phi)$. For any $X \in T M^{n}$ we put

$$
\phi X=P X+Q X
$$

where $P X$ and $Q X$ denotes the tangent and normal components of $\phi X$.

It was proved by M. Crasmareanu and C. Hretcanu in [58] that an almost product structure $F$ on a Riemannian manifold $(\widetilde{M}, \tilde{g})$ induces a Golden structure $\phi$ given by $\phi=\frac{1}{2}(I+\sqrt{5} F)$. Conversely, any golden structure $\phi$ on $\widetilde{M}$ induces an almost product structure $F=\frac{1}{\sqrt{5}}(2 \phi-I)$. Consequently, every locally product $M^{p}\left(c_{p}\right) \times M^{q}\left(c_{q}\right)$ of two real space forms $M^{p}\left(c_{p}\right)$ and $M^{q}\left(c_{q}\right)$ of constant sectional curvature $c_{p}$ and $c_{q}$ is a golden Riemannian manifold, which is called a locally product golden space form.

The Riemannian curvature tensor $\widetilde{R}$ of a locally product golden space form $M^{p}\left(c_{p}\right) \times M^{q}\left(c_{q}\right)$ is derived by N. Ö Poyraz and E. Yaşar [113] as follows:

$$
\begin{aligned}
\widetilde{R}(X, Y) Z= & \left(\frac{(\psi-1) c_{p}+\psi c_{q}}{2 \sqrt{5}}\right)\{g(Y, Z) X-g(X, Z) Y+g(\phi Y, Z) \phi X-g(\phi X, Z) \phi Y\} \\
& -\left(\frac{(1-\psi) c_{p}+\psi c_{q}}{4}\right)\{g(\phi Y, Z) X-g(\phi X, Z) Y+g(Y, Z) \phi X-g(X, Z) \phi Y\}
\end{aligned}
$$

where $\psi=\frac{1+\sqrt{5}}{2}$ is the golden proportion.

\subsection{C-totally real submanifolds of a locally Golden product space form}

A submanifold $M^{n}$ of a Golden Riemannian manifold $(\widetilde{M}, \tilde{g}, \phi)$ is called a C-totally real submanifold if $\phi$ maps each tangent space of $M^{n}$ into the correspondent normal space, i.e., $\phi\left(T_{x} M^{n}\right) \subset T_{x}^{\perp} M^{n}$ for $x \in M^{n}$.

The following result on $C$-totally real submanifolds of a golden product space form is obtained by M. A. Choudhary, O. Bahadir and H. Alsulami in [57].

Theorem 12.1. Let $M^{n}$ be an $n$-dimensional $C$-totally real submanifold of a locally product golden space form $\widetilde{M}^{m}=\left(M^{p}\left(c_{p}\right) \times M^{q}\left(c_{q}\right), \tilde{g}, \phi\right)$. Then

$$
\rho^{\perp} \leq\|H\|^{2}-2 \rho-2\left(\frac{(1-\psi) c_{p}-\psi c_{q}}{2 \sqrt{5}}\right)
$$

where $\psi=\frac{1+\sqrt{5}}{2}$ is the golden proportion.

Moreover, the equality holds in (12.3) if and only if there exists an orthonormal frame $\left\{e_{1}, \ldots, e_{n}, \xi_{1}, \ldots, \xi_{m-n}\right\}$ such that with respect to this frame the shape operators take the form of (7.4).

\subsection{Slant and invariant submanifolds of a locally Golden product space form}

A submanifold $M^{n}$ of a golden Riemannian manifold $(\widetilde{M}, \tilde{g}, \phi)$ is called a slant submanifold if, for each $0 \neq X \in T_{x} M^{n}, x \in M^{n}$, the angle $\theta(X)$ between $\phi X$ and $T_{x} M^{n}$ is constant, that is, $\theta(X)$ is independent of the choice of $x \in M^{n}$ and of $X \in T_{x} M^{n}$. If the slant angle $\theta$ of a slant submanifold $M^{n}$ satisfies $\theta=0$ (resp., $\theta=\frac{\pi}{2}$ ), then $M^{n}$ is called $\phi$-invariant (resp., $\phi$-anti-invariant). A slant submanifold which is neither invariant nor anti-invariant is called proper slant.

For proper slant submanifolds of a golden product space form, Choudhary, Bahadir and Alsulami proved the following result in [57]. 
Theorem 12.2. Let $M^{n}$ be an $n$-dimensional proper $\theta$-slant submanifold of a locally product golden space form $\bar{M}^{m}=\left(M^{p}\left(c_{p}\right) \times M^{q}\left(c_{q}\right), \tilde{g}, \phi\right)$. Then

$$
\begin{aligned}
\rho^{\perp} \leq & \|H\|^{2}-2 \rho-2\left(\frac{(1-\psi) c_{p}-\psi c_{q}}{2 \sqrt{5}}\right)\left\{1+\frac{\operatorname{trace}^{2} \phi}{n(n-1)}\right\} \\
& +2\left(\cos ^{2} \theta\right)\left(\frac{(1-\psi) c_{p}-\psi c_{q}}{2 \sqrt{5}}\right)\left\{\frac{1}{n-1}+\frac{\operatorname{trace} P}{n(n-1)}\right\}-\left(\frac{(1-\psi) c_{p}+\psi c_{q}}{4}\right) \frac{4}{n} \operatorname{trace} \phi .
\end{aligned}
$$

For invariant submanifolds of a golden product space form, Choudhary, Bahadir and Alsulami [57] proved the following.

Theorem 12.3. Let $M^{n}$ be an $n$-dimensional invariant submanifold of a locally product golden space form $\bar{M}^{m}=$ $\left(M^{p}\left(c_{p}\right) \times M^{q}\left(c_{q}\right), \tilde{g}, \phi\right)$. Then

$$
\begin{aligned}
\rho^{\perp} \leq & \|H\|^{2}-2 \rho-2\left(\frac{(1-\psi) c_{p}-\psi c_{q}}{2 \sqrt{5}}\right)\left\{1+\frac{\operatorname{trace}^{2} \phi}{n(n-1)}\right\} \\
& +2\left(\frac{(1-\psi) c_{p}-\psi c_{q}}{2 \sqrt{5}}\right)\left\{\frac{1}{n-1}+\frac{\operatorname{trace} P}{n(n-1)}\right\}-\left(\frac{(1-\psi) c_{p}+\psi c_{q}}{4}\right) \frac{4}{n} \operatorname{trace} \phi
\end{aligned}
$$

\section{Wintgen ideal submanifolds for statistical submanifolds}

The notion of statistical manifolds was introduced by S. Amari [3] in 1985, which provided a setting for the field of information geometry and it also associates a dual connection (known as conjugate connection). The nice applications of statistical manifolds in applied science and engineering have attracted the attention of many geometers. The theory of statistical model as statistical manifold is a fast growing research subject in differential geometry. Many articles have been published in the setting of statistical manifold in recent years.

\subsection{Statistical manifolds}

Let $(\widetilde{M}, \tilde{g})$ be a Riemannian manifold with Levi-Civita connection $\widetilde{\nabla}^{0}$. For a torsion-free affine connection $\widetilde{\nabla}$ on $(\widetilde{M}, \tilde{g})$, let $\widetilde{\nabla}^{*}$ be the torsion-free connection defined by

$$
Z \tilde{g}(X, Y)=\tilde{g}\left(\widetilde{\nabla}_{Z} X, Y\right)+\tilde{g}\left(X, \widetilde{\nabla}_{Z}^{*} Y\right)
$$

which is called the dual connection of $\widetilde{\nabla}$ with respect to $\tilde{g}$. It is easily shown that $\left(\widetilde{\nabla}^{*}\right)^{*}=\widetilde{\nabla}$. The Riemannian manifold $(\widetilde{M}, \tilde{g})$ equipped with a such pair of torsion-free affine connections $\widetilde{\nabla}, \widetilde{\nabla}^{*}$ is called a statistical manifold. And the pair $(\widetilde{\nabla}, \tilde{g})$ is called a statistical structure on $\tilde{M}$. If $(\widetilde{\nabla}, \tilde{g})$ is a statistical structure on $\widetilde{M}$, then $\left(\widetilde{\nabla}^{*}, \tilde{g}\right)$ is also a statistical structure.

For the statistical manifold, we have

$$
\widetilde{\nabla}+\widetilde{\nabla}^{*}=2 \widetilde{\nabla}^{0}
$$

In particular, $(\widetilde{M}, \widetilde{\nabla}, \tilde{g})$ is called a trivial statistical manifold whenever $\widetilde{\nabla}=\widetilde{\nabla}^{*}$.

A statistical structure $(\widetilde{\nabla}, \tilde{g})$ is said to be of constant curvature $c$ if

$$
\widetilde{R}^{\widetilde{\nabla}}(X, Y) Z=c\{\tilde{g}(Y, X) X-\tilde{g}(X, Z) Y\}
$$

holds, where $\widetilde{R} \widetilde{\nabla}$ denotes the curvature tensor associated with $\widetilde{\nabla}$. A statistical structure $(\widetilde{\nabla}, \tilde{g})$ of constant curvature 0 is called a Hessian structure (cf. e.g., [50, 76]).

Since the curvature tensor $\widetilde{R}^{\widetilde{\nabla}}$ and $\widetilde{R}^{\widetilde{\nabla}^{*}}$ of the dual connections $\widetilde{\nabla}$ and $\widetilde{\nabla}{ }^{*}$ on $\widetilde{M}$ satisfy

$$
g\left(\widetilde{R}^{\widetilde{\nabla}^{*}}(X, Y) Z, W\right)=-\tilde{g}\left(Z, \widetilde{R}^{\widetilde{\nabla}}(X, Y) W\right),
$$

it follows that if $(\widetilde{\nabla}, \tilde{g})$ is a statistical structure of constant curvature $c$, then $\left(\widetilde{\nabla}^{*}, \tilde{g}\right)$ is also a statistical structure of constant curvature $c$. In particular, if $(\widetilde{\nabla}, \tilde{g})$ is Hessian, then $\left(\widetilde{\nabla}^{*}, \tilde{g}\right)$ is also Hessian (see [123]). 


\subsection{Statistical submanifolds in statistical manifolds}

Let $M^{n}$ be an $n$-dimensional submanifold of a statistical $m$-manifold $\left(\widetilde{M}^{m}, \tilde{g}\right)$, then $\left(M^{n}, g\right)$ is also a statistical manifold with the induced connection $\nabla$ and the induced metric $g$. The fundamental equations for statistical submanifolds have been derived by P. W. Vos [133] in 1989 as follows. For tangent vector fields $X, Y$ of $M^{n}$ the Gauss formulas are

$$
\widetilde{\nabla}_{X} Y=\nabla_{X} Y+h(X, Y), \quad \widetilde{\nabla}_{X}^{*} Y=\nabla_{X}^{*} Y+h^{*}(X, Y),
$$

where $h, h^{*}$ are symmetric and bilinear, called the second fundamental forms (see [10]) or the imbedding curvature tensors (see [65]). We put

$$
h^{0}=\frac{1}{2}\left(h+h^{*}\right) .
$$

Since $h$ and $h^{*}$ are bilinear, there exist linear transformations $A_{\xi}$ and $A_{\xi}^{*}$ on $T M^{n}$, known as the shaper operators, defined by

$$
g\left(A_{\xi} X, Y\right)=\tilde{g}(h(X, Y), \xi), \quad g\left(A_{\xi}^{*} X, Y\right)=\tilde{g}\left(h^{*}(X, Y), \xi\right),
$$

for any normal vector field $\xi$ of $M^{n}$. Further, the corresponding Weingarten formulas of $M^{n}$ are given by

$$
\widetilde{\nabla}_{X} \xi=-A_{\xi} X+\nabla_{X}^{\perp} \xi, \quad \widetilde{\nabla}_{X}^{*} \xi=-A_{\xi}^{*} X+\nabla_{X}^{* \perp} \xi .
$$

Let $\left\{e_{1}, \ldots, e_{n}\right\}$ and $\left\{\xi_{1}, \ldots, \xi_{m-n}\right\}$ be orthonormal tangent and normal frames on $M^{n}$, respectively. Then the corresponding mean curvature vector fields $H$ and $H^{*}$ are given respectively by

$$
H=\frac{1}{n} \sum_{i=1}^{n} h\left(e_{i}, e_{i}\right)=\frac{1}{n} \sum_{r=1}^{m-n}\left(\sum_{i=1}^{n} h_{i i}^{r}\right) \xi_{r}, \quad H^{*}=\frac{1}{n} \sum_{i=1}^{n} h^{*}\left(e_{i}, e_{i}\right)=\frac{1}{n} \sum_{r=1}^{m-n}\left(\sum_{i=1}^{n} h_{i i}^{* r}\right) \xi_{r},
$$

for $1 \leq i, j \leq n$ and $1 \leq r \leq m-n$, where $h_{i j}^{r}=\tilde{g}\left(h\left(e_{i}, e_{j}\right), \xi_{r}\right)$ and $h_{i j}^{* r}=\tilde{g}\left(h^{*}\left(e_{i}, e_{j}\right), \xi_{r}\right)$.

Let $\widetilde{R}$ and $R$ be the curvature tensor of $\tilde{\nabla}$ and $\nabla$, respectively. Then the corresponding Gauss, Codazzi and Ricci equations are given respectively by [133]

$$
\begin{aligned}
& \tilde{g}(\widetilde{R}(X, Y) Z, W)=g(R(X, Y) Z, W)+\tilde{g}\left(h(X, Z), h^{*}(Y, W)\right)-\tilde{g}\left(h^{*}(X, W), h(Y, Z)\right), \\
& (\widetilde{R}(X, Y) Z)^{\perp}=\nabla_{X}^{\perp} h(Y, Z)-h\left(\nabla_{X} Y, Z\right)-h\left(Y, \nabla_{X} Z\right)-\left\{\nabla_{Y}^{\perp} h(X, Z)-h\left(\nabla_{Y} X, Z\right)-h\left(X, \nabla_{Y} Z\right)\right\}, \\
& \tilde{g}\left(\widetilde{R}^{\perp}(X, Y) \xi, \eta\right)=\tilde{g}(R(X, Y) \xi, \eta)+g\left(\left[A_{\xi}^{*}, A_{\eta}\right] X, Y\right),
\end{aligned}
$$

for any $X, Y, Z, W$ tangent to $M^{n}$ and $\xi, \eta$ normal to $M^{n}$, where $\widetilde{R}^{\perp}$ is the Riemannian curvature tensor on the normal bundle $T^{\perp} M^{n}$.

Similarly, let $\widetilde{R}^{*}$ and $R^{*}$ denote respectively the curvature tensor fields with respect to $\widetilde{\nabla}^{*}$ and $\nabla^{*}$. We can obtain the duals of all equations (13.4)-(13.6) with respect to $\widetilde{\nabla}^{*}$ and $\nabla^{*}$. Also,

$$
\tilde{S}=\frac{1}{2}\left(\widetilde{R}+\widetilde{R}^{*}\right) \text { and } S=\frac{1}{2}\left(R+R^{*}\right)
$$

are respectively the curvature tensor fields of $\widetilde{M}^{m}$ and $M^{n}$. Thus, the sectional curvature $K^{\nabla, \nabla^{*}}$ on $M^{n}$ of $\widetilde{M}^{m}$ is defined by $[107,108]$

$$
K^{\nabla, \nabla^{*}}(X \wedge Y)=g(S(X, Y) Y, X)=\frac{1}{2}\left\{g(R(X, Y) Y, X)+g\left(R^{*}(X, T) Y, X\right)\right\}
$$

for any orthonormal vectors $X, Y \in T_{x} M^{n}, x \in M^{n}$.

Let $\left\{e_{1}, \ldots, e_{n}\right\}$ and $\left\{\xi_{1}, \ldots, \xi_{m-n}\right\}$ be respectively orthonormal basis of $T_{p} M^{n}$ and $T_{p}^{\perp} M^{n}$ for $x \in M^{n}$. Then the normalized scalar curvature of $M^{n}$ is defined as

$$
\rho=\frac{1}{n(n-1)} \sum_{1 \leq i<j \leq n}\left\{g\left(R\left(e_{i}, e_{j}\right) e_{j}, e_{i}\right)+g\left(R^{*}\left(e_{i}, e_{j}\right) e_{j}, e_{i}\right)\right\}
$$

The normalized normal scalar curvature of $M^{n}$ in $\widetilde{M}^{m}$ is defined as

$$
\rho^{\perp}=\frac{1}{n(n-1)}\left\{\sum_{1 \leq r<s \leq m-n} \sum_{1 \leq i<j \leq n} g\left(R^{\perp}\left(e_{i}, e_{j}\right) \xi_{r}, \xi_{s}\right)+g\left(R^{* \perp}\left(e_{i}, e_{j}\right) \xi_{r}, \xi_{s}\right)\right\}^{1 / 2} .
$$




\subsection{Wintgen type inequalities of statistical submanifolds in statistical space forms}

Let $\widetilde{M}^{m}(c)$ be an $m$-dimensional statistical manifold of constant curvature $c$. Consider a statistical surface $\left(M^{2}, \nabla\right)$ of the statistical 4-manifold $\widetilde{M}^{4}(c)$. Denote the Gauss curvature and the normal curvature of $M^{2}$ with respect to $\nabla$ by $G$ and $G^{\perp}$, respectively, and denote the Gauss curvature with respect to the Levi-Civita connection $\nabla^{0}$ by $G^{0}$. Similarly, we denote the mean vector field, the dual mean curvature and the sectional curvature with respect to the Levi-Civita connection by $H, H^{*}$ and $\widetilde{K}^{0}$, respectively.

The following Wintgen type inequality for statistical surfaces in 4-dimensional statistical space forms was obtained by M. E. Aydin and I. Mihai in [7].

Theorem 13.1. Let $M^{2}$ be a statistical surface in a 4-dimensional statistical space form $\widetilde{M}^{4}(c)$ of constant curvature $c$. Then

$$
G+\left|G^{\perp}\right|+2 G^{0} \leq \frac{1}{2}\left(\|H\|^{2}+\left\|H^{*}\right\|^{2}\right)-c+2 \tilde{K}^{0}\left(e_{1} \wedge e_{2}\right) .
$$

In particular, Theorem 13.1 implies the following result for $c=0$.

Corollary 13.1. Let $M^{2}$ be a statistical surface of a Hessian 4-dimensional statistical manifold $\widetilde{M}^{4}(c)$ of Hessian curvature 0 . Then

$$
G+\left|G^{\perp}\right|+2 G^{0} \leq \frac{1}{2}\left(\|H\|^{2}+\left\|H^{*}\right\|^{2}\right) .
$$

For higher dimensional submanifolds in statistical space forms, M. E. Aydin, A. Mihai, I. Mihai proved in [8] the following.

Theorem 13.2. Let $M^{n}$ be a submanifold in a statistical manifold $\widetilde{M}^{m}(c)$ of constant curvature $c$. Then

$$
\rho^{\perp}+3 \rho \leq \frac{15}{2}\left(\|H\|^{2}+\left\|H^{*}\right\|^{2}\right)+12 g\left(H, H^{*}\right)-3 c+30\left(\tilde{\rho}^{0}-\rho^{0}\right),
$$

where

$$
\tilde{\rho}^{0}=\frac{2}{n(n-1)} \sum_{1 \leq i<j \leq n} \tilde{R}^{0}\left(e_{i}, e_{j}, e_{i}, e_{j}\right), \quad \rho^{0}=\tilde{\rho}^{0}+\frac{2}{n(n-1)} \sum_{r=1}^{m-n} \sum_{1 \leq i<j \leq n}\left[h_{i i}^{0 r} h_{j j}^{0 r}-\left(h_{i j}^{0 r}\right)^{2}\right] .
$$

\subsection{Totally real statistical submanifolds in holomorphic statistical space forms}

Let $\bar{M}^{m}$ be a Kaehler manifold of complex dimension $m$ equipped with an almost complex structure $J$ and a Kaehlerian metric $g$. A quadruple $\left(\bar{M}^{m}, \bar{\nabla}, g, J\right)$ is called a holomorphic statistical manifold (see, e.g., [125, 127]) if

(a) $(\bar{\nabla}, g)$ is a statistical structure on $\bar{M}^{m}$ and

(b) the fundamental 2-form $\omega$ on $\bar{M}^{m}$ given by $\omega(X, Y)=g(X, J Y)$ is $\bar{\nabla}$-parallel, that is, $\bar{\nabla} \omega=0$.

A statistical submanifold $M^{n}$ of a holomorphic statistical manifold $\bar{M}^{m}$ is said to be totally real if the almost complex structure $J$ of $\bar{M}^{m}$ carries each tangent space of $M^{n}$ into its corresponding normal space. A totally real statistical submanifold of maximal dimension is called Lagrangian statistical submanifold.

For Lagrangian statistical submanifolds in a holomorphic statistical space form $\bar{M}^{m}(c)$, A. N. Siddiqui, N. Aliya and M. H. Shahid proved the following two results in [127].

Theorem 13.3. Let $M^{n}$ be a Lagrangian statistical submanifold in a holomorphic statistical manifold $\bar{M}^{m}(c)$ of constant holomorphic sectional curvature $c$. Then

$$
n(n-1)\left(\rho^{\perp}\right)^{2}+\frac{c}{n(n-1)}\left(\|H\|^{2}+\left\|H^{*}\right\|^{2}\right) \geq\left(\frac{2 \rho}{n(n-1)}-\frac{c}{2}\right)^{2}+\frac{4 c}{n(n-1)}\left\|H^{0}\right\|^{2} .
$$

Theorem 13.4. Let $M^{n}$ be a Lagrangian statistical submanifold in a holomorphic statistical manifold $\bar{M}^{n}(c)$ of constant holomorphic sectional curvature $c$. Then

$$
\rho \geq \frac{c}{4}+\frac{n}{n-1} g\left(H, H^{*}\right)-\frac{1}{n(n-1)}\|h\| \cdot\left\|h^{*}\right\| .
$$




\subsection{Statistical submanifolds of statistical manifolds with quasi-constant curvature}

The notion of a quasi-constant curvature Riemannian manifold was introduced by the author and K. Yano in [55] as a Riemannian manifold $(\widetilde{M}, g)$ equipped with a unit vector field $P$ such that its curvature tensor $\widetilde{R}$ satisfies the condition:

$$
\begin{aligned}
\widetilde{R}(X, Y) Z= & a[\tilde{g}(Y, Z) X-\tilde{g}(X, Z) Y]+b\{T(Y) T(Z) X-\tilde{g}(X, Z) T(Y) P \\
& +\tilde{g}(Y, Z) T(X) P-T(X) T(Z) Y\},
\end{aligned}
$$

where $a, b$ are scalar functions and $T$ is a 1-form defined by the musical isomorphism: $T(X)=g(X, P)$. Similarly, a statistical structure $(\tilde{\nabla}, \tilde{g})$ on $\bar{M}^{m}$ is said to be of quasi-constant curvature if the curvature tensor $\widetilde{R}$ of $\tilde{\nabla}$ satisfies the same condition (13.7) (see [9]). In particular, if $b=0$, then $\bar{M}^{m}$ is simply a statistical manifold of constant curvature.

In [9], H. Aytimur, C. Ozgur proved the following Wintgen type inequality for statistical submanifolds of a statistical manifold with quasi-constant curvature.

Theorem 13.5. Let $M^{n}$ be a statistical submanifold of a statistical manifold $\bar{M}^{m}$ of quasi-constant curvature. Then we have

$$
\rho^{\perp} \leq \frac{3}{2}\left(\|H\|^{2}+\left\|H^{*}\right\|^{2}\right)+24\left\|H^{0}\right\|^{2}+3 \rho-3 a-\frac{6}{n} b+30\left(\tilde{\rho}^{0}-\rho^{0}\right),
$$

where $\tilde{\rho}^{0}$ and $\rho^{0}$ denote the normalized scalar curvatures of the Levi-Civita connection $\tilde{\nabla}^{0}$ and the induced Levi-Civita connection $\nabla^{0}$, respectively, and $\rho^{\perp}$ is the normalized normal scalar curvature of the statistical manifold $\left(M^{n}, \nabla, g\right)$.

\subsection{Wintgen type inequalities for submanifolds in statistical warped products}

Let $B$ and $F$ be two Riemannian manifolds with Riemannian metrics $g_{B}$ and $g_{F}$, respectively, and $f$ be a positive differentiable function on $B$. The warped product $B \times_{f} F$ is the product manifold $B \times F$ equipped with the Riemannian metric $g=g_{B}+f^{2} g_{F}$. The function $f$ is called the warping function. A warped product is said to be proper if its warping function is non-constant. In [131], L. Todjihounde provided a method to equip a dualistic structure on the warped product manifold $B \times{ }_{f} F$.

In [105], C. Murathan and B. Şahin investigated statistical submanifolds $M^{n}$ of a statistical warped product $I \times_{f} \widetilde{M}^{m}(c)$, where $\widetilde{M}^{m}(c)$ is a statistical manifold of constant curvature $c$ so that the metric of $I \times_{f} \widetilde{M}^{m}(c)$ is given by

$$
g=d t^{2}+f(t)^{2} g_{\widetilde{M}^{m}(c)},
$$

where $g_{\widetilde{M}^{m}(c)}$ is the metric of $\widetilde{M}^{m}(c)$.

In [105], they proved the following Wintgen type inequality for such submanifolds.

Theorem 13.6. Let $M^{n}$ be a statistical submanifold of a statistical warped product $I \times_{f} \widetilde{M}^{m}(c)$, where $\widetilde{M}^{m}(c)$ is a statistical manifold of constant curvature $c$. Then

$$
\rho^{\perp} \leq-12 \rho^{0}+3 \rho+9\left(\frac{c}{f^{2}}-\frac{f^{\prime 2}}{f^{2}}\right)\left(1-\frac{2}{n}\|T\|^{2}\right)-\frac{18 f^{\prime \prime}}{n f}\|T\|^{2}+6\left\|H^{0}\right\|^{2}+\frac{3}{2}\left(\|H\|^{2}+\left\|H^{*}\right\|^{2}\right),
$$

where $T=\partial / \partial t-\sum_{r=1}^{m-n} a_{r} \xi_{r}$ is a tangent vector field of $M^{n}$ and $\left\{\xi_{1}, \ldots, \xi_{m-n}\right\}$ is a local orthonormal frame of the normal bundle $T^{\perp} M^{n}$.

\subsection{Wintgen type inequality for Legendrian submanifolds of Sasakian statistical manifolds}

If a statistical manifold $\left(\widetilde{M}^{m}, \tilde{g}, \widetilde{\nabla}\right)$ has an almost contact metric-like structure $(\varphi, \xi, \eta)$, then $\left(\widetilde{M^{m}}, \tilde{g}, \widetilde{\nabla}, \varphi, \xi, \eta\right)$ is known as almost contact metric-like statistical manifold. In particular, if $(\varphi, \xi, \eta)$ is a Sasakian structure, then $\left(\widetilde{M}^{m}, \tilde{g}, \widetilde{\nabla}, \varphi, \xi, \eta\right)$ is called Sasakian statistical manifold. Further, a Sasakian statistical manifold is called a Sasakian statistical space form if it has constant $\varphi$-sectional curvature.

In [19], M. N. Boyom, Z. Jabeen, M. A. Lone, M. S. Lone and M. H. Shahid proved the following Wintgen type inequality for Legendrian submanifold in Sasakian statistical space forms.

Theorem 13.7. Let $M^{n}$ be a Legendrian submanifold of a Sasakian statistical space form of constant $\varphi$-sectional curvature $c$. Then we have

$$
\rho^{\perp} \leq 6\left(\|H\|^{2}+\left\|H^{*}\right\|^{2}+16\left\|H^{0}\right\|^{2}\right)-12 \rho-9+3 c+120\left(\bar{\rho}-\rho^{0}\right) .
$$




\subsection{Statistical submanifolds of Kenmotsu statistical manifolds}

For a statistical structure $(\widetilde{\nabla}, \tilde{g})$ on a manifold $\widetilde{M}$, put $\widetilde{K}=\widetilde{\nabla}-\nabla^{0}$. Then $\widetilde{K}$ satisfies (cf. [65]).

$$
\widetilde{K}_{X} Y=\widetilde{K}_{Y} X, \quad \tilde{g}\left(K_{X} Y, Z\right)=\tilde{g}\left(Y, K_{X} Z\right) .
$$

Let $(\widetilde{\mathcal{K}}, \tilde{g}, \tilde{\varphi}, \tilde{\xi}, \tilde{\eta})$ be a Kenmotsu manifold and assume that $(\widetilde{\nabla}, \tilde{g})$ is a statistical structure on $\widetilde{\mathcal{K}}$. Then the quadruple $(\widetilde{\nabla}, \tilde{g}, \tilde{\varphi}, \tilde{\xi})$ is called a Kenmotsu statistical structure on $\tilde{M}$ if

$$
\widetilde{K}(X, \tilde{\varphi} Y)+\tilde{\varphi} \widetilde{K}(X, Y)=0 .
$$

holds for any vector fields $X, Y$ tangent to $\widetilde{\mathcal{K}}$. A manifold equipped with a Kenmotsu statistical structure is called a Kenmotsu statistical manifold. A Kenmotsu statistical manifold $(\widetilde{\mathcal{K}}, \tilde{g}, \tilde{\varphi}, \tilde{\xi}, \tilde{\eta})$ is called a Kenmotsu statistical space form if it has constant $\tilde{\varphi}$-sectional curvature.

For a statistical submanifold $M^{n}$ of a Kenmotsu statistical manifold $\mathcal{K}^{2 m+1}$, the Carasoti curvatures $\mathcal{C}$ and $\mathcal{C}^{*}$ of $M^{n}$ are defined respectively by

$$
\mathcal{C}=\frac{1}{n} \sum_{1 \leq i<j \leq n} \sum_{s=1}^{2 m+1-n}\left(h_{i j}^{s}\right)^{2}=\frac{\|h\|^{2}}{n}, \quad \mathcal{C}^{*}=\frac{1}{n} \sum_{1 \leq i<j \leq n} \sum_{s=1}^{2 m+1-n}\left(h_{i j}^{* s}\right)^{2}=\frac{\left\|h^{*}\right\|^{2}}{n} .
$$

For statistical submanifolds of a Kenmotsu statistical space form, P. Bansal, S. Uddin and M. H. Shahid proved the following Wintgen type inequality.

Theorem 13.8. [11] Let $M^{n}$ be a statistical submanifolds of a Kenmotsu statistical space form $\mathcal{K}^{2 m+1}(c)$ of constant $\varphi$-sectional curvature $c$. Then

$$
\begin{aligned}
\rho^{\perp}-\sqrt{3} \rho \leq & \frac{5 \sqrt{3}}{2}\left(\|H\|^{2}+\left\|H^{*}\right\|^{2}\right)+4 \sqrt{3} g\left(H, H^{*}\right)-\sqrt{3}\left(\frac{c-3}{2}\right)-\frac{20 \sqrt{3}}{n(n-1)} \sum_{1 \leq i<j \leq n} \sum_{s=1}^{2 m+1-n}\left[h_{i i}^{0 s} h_{j j}^{0 s}-\mathcal{C}^{0}\right] \\
& -\left(\frac{\sqrt{3}(c+1)}{2 n(n-1)}\right)\left(3\|\varphi\|^{2}-2(n-1)\right),
\end{aligned}
$$

where $\mathcal{C}^{0}=\frac{1}{2}\left(\mathcal{C}+\mathcal{C}^{*}\right)$ and $h_{i j}^{0 s}=\frac{1}{2}\left(h_{i j}^{s}+h_{i j}^{* s}\right)$.

In particular, if the statistical submanifold $M^{n}$ is totally geodesic with respect to $\nabla^{0}$, then Theorem 13.8 implies the following.

Corollary 13.2. Let $M^{n}$ be a statistical submanifolds of a Kenmotsu statistical space form $\mathcal{K}^{2 m+1}(c)$ such that $M^{n}$ is totally geodesic with respect to $\nabla^{0}$. Then

$$
\rho^{\perp}-\sqrt{3} \rho \leq \frac{5 \sqrt{3}}{2}\left(\|H\|^{2}+\left\|H^{*}\right\|^{2}\right)+4 \sqrt{3} g\left(H, H^{*}\right)-\sqrt{3}\left(\frac{c-3}{2}\right)-\left(\frac{\sqrt{3}(c+1)}{2 n(n-1)}\right)\left(3\|\varphi\|^{2}-2(n-1)\right) .
$$

One may consider the local version of any Kenmotsu manifold. Let $\left(M_{0}, g_{0}, J\right)$ be an almost Hermitian manifold (see §10.4). Let us consider $\bar{N}=M_{0} \times \mathbb{R}, \tilde{g}=e^{2 t} g_{0}+(d t)^{2}$, the structure vector field $\xi=\frac{\partial}{\partial t}$, and the structure tensor field $\varphi$ on $\bar{N}$. Then we have:

(1) The triple $(\tilde{g}, \varphi, \xi)$ is an almost contact metric structure on $\bar{N}$.

(2) The pair $(g, J)$ is a Kaehler structure on $M_{0}$ if and only if the triple $(\tilde{g}, \varphi, \xi)$ is a Kenmotsu structure on $\bar{N}$.

Remark 13.1. Let $\bar{N}=M_{0} \times \mathbb{R}$ with a statistical structure $\left(\widetilde{\nabla}=\nabla^{\tilde{g}}+\tilde{\mathcal{K}}, \tilde{g}\right)$. If

$$
\left(\bar{N}=M_{0} \times \mathbb{R}, \tilde{\nabla}=\nabla^{\tilde{g}}+\tilde{\mathcal{K}}, \tilde{g}, \varphi, \xi\right)
$$

is a Kenmotsu statistical manifold of constant $\varphi$-sectional curvature $c$, then $c=-1$ and $\left(M_{0}, \nabla=\nabla^{g_{0}}+\mathcal{K}, g_{0}, J\right)$ is a Kenmotsu statistical manifold of constant holomorphic sectional curvature 0 (see [11, Remark 4]).

In [11], P. Bansal, S. Uddin and M. H. Shahid also obtained the following. 
Theorem 13.9. Let $\left(\bar{N}=M_{0} \times \mathbb{R}, \widetilde{\nabla}=\nabla^{\tilde{g}}+\tilde{K}, \tilde{g}, \varphi, \xi\right)$ be the Kenmotsu statistical space form of constant $\varphi$-sectional curvature c as given in Remark 13.1. If $M^{n}$ is a statistical submanifolds of $\bar{N}$, then

$$
\rho^{\perp}-\sqrt{3} \rho \leq \frac{5 \sqrt{3}}{2}\left(\|H\|^{2}+\left\|H^{*}\right\|^{2}\right)+4 \sqrt{3} g\left(H, H^{*}\right)+2 \sqrt{3}-\frac{20 \sqrt{3}}{n(n-1)} \sum_{1 \leq i<j \leq n} \sum_{s=1}^{2 m+1-n}\left[h_{i i}^{0 s} h_{j j}^{0 s}-\mathcal{C}^{0}\right] .
$$

In particular, if the statistical submanifold $M^{n}$ is totally geodesic with respect to $\nabla^{0}$, then Theorem 13.9 implies the following.

Corollary 13.3. Let $\left(\bar{N}=M_{0} \times \mathbb{R}, \widetilde{\nabla}=\nabla^{\tilde{g}}+\tilde{K}, \tilde{g}, \varphi, \xi\right)$ be the Kenmotsu statistical space form of constant $\varphi$-sectional curvature c as given in Remark 13.1. If a statistical submanifold $M^{n}$ is totally geodesic in $\bar{N}$ with respect to $\nabla^{0}$, then

$$
\rho^{\perp}-\sqrt{3} \rho \leq \frac{5 \sqrt{3}}{2}\left(\|H\|^{2}+\left\|H^{*}\right\|^{2}\right)+4 \sqrt{3} g\left(H, H^{*}\right)+2 \sqrt{3} .
$$

\subsection{Legendrian submanifolds of almost Kenmotsu statistical manifolds}

Let $\left(\widetilde{M}, \widetilde{g}, \widetilde{\nabla}, \widetilde{\nabla}^{*}\right)$ be a statistical manifold. If $\widetilde{M}$ is an almost contact metric manifold, then $\widetilde{M}$ is called almost contact metric statistical manifold; and if $(\widetilde{M}, \widetilde{g}, J)$ is an almost Hermitian manifold, then $(\widetilde{M}, \widetilde{g}, J, \widetilde{\nabla}, \widetilde{\nabla} *)$ is called almost Hermitian statistical manifold. In particular, if $(\widetilde{M}, \widetilde{g}, J)$ is an (almost) Kaehler manifold, then $\left(\widetilde{M}, \widetilde{g}, J, \widetilde{\nabla}, \widetilde{\nabla}^{*}\right)$ is called (almost) Kaehler statistical manifold.

Let $(\widetilde{N}, \nabla, g, J)$ be an almost Hermitian statistical manifold and let $\left(\mathbb{R}, d t,{ }^{\mathbb{R}} \nabla\right)$ be trivial statistical manifold. Consider the warped product $\widetilde{M}=\mathbb{R} \times{ }_{f} \widetilde{N}$ with warping function $f>0$ and with the warped product metric

$$
\tilde{g}=d t^{2}+f^{2} f_{\widetilde{N}} .
$$

Let $\xi=\frac{\partial}{\partial t}$ be the structure vector field on $\widetilde{M}$ and put $\eta=d t$. Then, for an arbitrary vector field $X$ on $\widetilde{M}$, we may put

$$
X=\tilde{X}-\eta(\widetilde{X}) \xi .
$$

By applying the almost complex structure $J$, we may define a tensor field $\varphi$ of type $(1,1)$ on $\widetilde{M}$ given by $\varphi \widetilde{X}=J X$ for any tangent vector field $\widetilde{X}$ on $\widetilde{M}$. Now, it is direct to verify that $(\widetilde{M}, \tilde{g}, \varphi, \xi, \eta)$ is an almost contact metric manifold. It was proved in [82, Theorem 4.1] that $(\tilde{N}, \nabla, g, J)$ is an almost Kaehler statistical manifold if and only if the warped product $\left(\widetilde{M}=\mathbb{R} \times_{f} \tilde{N}, \tilde{g}, \varphi, \xi\right)$ is an almost $(-\ln f)^{\prime}$-Kenmotsu statistical manifold.

For Legendrian submanifold of the statistical warped product manifolds $\widetilde{M}=\mathbb{R} \times_{f} \tilde{N}(c), \mathrm{R}$. Goriunus, I. K. Erken, A. Yazla and C. Murathan [82] the following.

Theorem 13.10. Let $\left(\mathbb{R}, d t,{ }^{\mathbb{R}} \nabla\right)$ be a trivial statistical manifold and $\widetilde{N}(c)$ be a holomorphic statistical space form. If $M^{n}$ is a Legendrian submanifold of the statistical warped product manifolds $\widetilde{M}=\mathbb{R} \times_{f} \widetilde{N}(c)$, then

$$
\rho^{\perp} \leq 2 \rho-8 \rho^{0}+\frac{1}{4 f^{2}}\left(2 f|c|-c+4\left(f^{\prime}\right)^{2}\right)+\|H\|^{2}+\left\|H^{*}\right\|^{2}+4\left\|H^{0}\right\|^{2} .
$$

Theorem 13.10 implies the following two corollaries.

Corollary 13.4. Let $\left(\mathbb{R}, d t,{ }^{\mathbb{R}} \nabla\right)$ be a trivial statistical manifold and $\widetilde{N}(0)=\mathbb{C}^{m}$ be a holomorphic statistical space form. If $M^{n}$ is a Legendrian submanifold of the statistical warped product manifolds $\widetilde{M}=\mathbb{R} \times e^{t} \mathbb{C}^{m}$, then

$$
\rho^{\perp} \leq 2 \rho-8 \rho^{0}+\|H\|^{2}+\left\|H^{*}\right\|^{2}+4\left\|H^{0}\right\|^{2}+1 .
$$

Corollary 13.5. Let $\left(\mathbb{R}, d t,{ }^{\mathbb{R}} \nabla\right)$ be a trivial statistical manifold and $\widetilde{N}(c)$ be a holomorphic statistical space form. If $M^{n}$ is a Legendrian submanifold of the statistical cosymplectic manifolds $\widetilde{M}=\mathbb{R} \times \widetilde{N}(c)$, then

$$
\rho^{\perp} \leq 2 \rho-8 \rho^{0}+\|H\|^{2}+\left\|H^{*}\right\|^{2}+4\left\|H^{0}\right\|^{2}+\frac{1}{4}(2|c|-c) .
$$




\section{Möbious geometry and Wintgen ideal submanifolds}

A diffeomorphism $\psi: S^{m} \rightarrow S^{m}$ which takes round $m$-spheres into itself is called a Möbius transformation. All Möbius transformations form a transformation group, called the Möbius transformation group of $S^{m}$, denoted by $\mathcal{M}\left(S^{m}\right)$. It is well known that the Möbius group $\mathcal{M}\left(S^{m}\right)$ coincides with the conformal group $\mathcal{C}\left(S^{n+1 m}\right)$ for $m \geq 2$. Möbius geometry has a long history. On the other hand, Möbius geometry of submanifolds was only studied extensively during the past 15 years. In particular, a complete Möbius invariant system for a submanifold $\varphi: M^{n} \rightarrow S^{m}$ was obtained by Changping Wang in [134]. Also, based on a result of F. Dillen, J. Fastenakels and J. Van der Veken in [72], M. Dajczer and R. Tojeiro [60] observed an important property that the Wintgen inequality, as well as the equality case, are invariant under Möbius transformations of the ambient space. Consequently, it is important to investigate Wintgen ideal submanifolds in the framework of Möbius geometry.

\subsection{Submanifold theory in Möbius geometry}

In this subsection, we provide the complete Möbius invariant system developed by Changping Wang in [134] as follows. The Lorentz inner product between $Y=\left(Y_{0}, Y_{1}, \cdots, Y_{n+p+1}\right)$ and $Z=\left(Z_{0}, Z_{1}, \cdots, Z_{n+p+1}\right) \in \mathbb{E}_{1}^{n+p+2}$ is given by

$$
\langle Y, Z\rangle=-Y_{0} Z_{0}+Y_{1} Z_{1}+\cdots+Y_{n+p+1} Z_{n+p+1} .
$$

Let $\phi: M^{n} \rightarrow S^{n+p} \subset \mathbb{E}^{n+p+1}$ be a submanifold without umbilical points. Take $\left\{e_{i}, \ldots, e_{n}\right\}$ as the tangent frame with respect to the induced metric $I=d \phi \cdot d \phi$, and $\left\{\theta_{1}, \ldots, \theta_{n}\right\}$ as the dual 1 -forms. Let $\left\{\hat{\xi}_{1}, \ldots, \hat{\xi}_{p}\right\}$ be orthonormal frame for the normal bundle.

The second fundamental form and the mean curvature of $\phi$ are

$$
I I=\sum_{i j, r} h_{i j}^{r} \theta_{i} \otimes \theta_{j} \hat{\xi}_{r}, \quad H=\frac{1}{n} \sum_{j, r} h_{j j}^{r} \hat{\xi}_{r}=\sum_{r} H^{r} \hat{\xi}_{r},
$$

respectively. Define the Möbius position vector $Y: M^{n} \rightarrow \mathbb{E}_{1}^{n+p+2}$ of $f$ by

$$
Y=\rho(1, \phi), \quad \rho=\sqrt{\frac{n}{n-1}}\left|I I-\frac{1}{n} \operatorname{trace}(I I) I\right|
$$

which is called the canonical lift of $\phi$.

Two submanifolds $\phi, \bar{\phi}: M^{n} \rightarrow S^{n+p}$ are said to be Möbius equivalent if there exists $T$ in the Lorentz group $O(n+p+1,1)$ in $\mathbb{E}_{1}^{n+p+2}$ such that $\bar{Y}=Y T$. It follows immediately that

$$
\mathrm{g}=\langle d Y, d Y\rangle=\rho^{2} d \phi \cdot d \phi
$$

is a Möbius invariant, called the Möbius metric of $\phi$. Denote by $\Delta$ the Laplacian with respect to g.

Define

$$
N=-\frac{1}{n} \Delta Y-\frac{1}{2 n^{2}}\langle\Delta Y, \Delta Y\rangle Y
$$

Let $\left\{E_{1}, \cdots, E_{n}\right\}$ be a local orthonormal frame for $\left(M^{n}, \mathrm{~g}\right)$ with dual 1-forms $\left\{\omega_{1}, \cdots, \omega_{n}\right\}$. We define the tangent frame $Y_{j}=E_{j}(Y)$ and the normal frame

$$
\xi_{r}=\left(H^{r}, \hat{\xi}_{r}+H^{r} \phi\right) .
$$

Then $\left\{Y, N, Y_{j}, \xi_{r}\right\}$ is a moving frame of $\mathbb{E}_{1}^{n+p+2}$ along $M^{n}$, which is orthonormal except

$$
\langle Y, Y\rangle=0=\langle N, N\rangle, \quad\langle N, Y\rangle=1 .
$$

Consider the range of indices as below: $1 \leq i, j, k \leq n ; 1 \leq r, s \leq p$. The structure equations are then given by

$$
\begin{aligned}
d Y & =\sum_{i} \omega_{i} Y_{i}, \\
d N & =\sum_{i j} A_{i j} \omega_{i} Y_{j}+\sum_{i, r} C_{i}^{r} \omega_{i} \xi_{r}, \\
d Y_{i} & =-\sum_{j} A_{i j} \omega_{j} Y-\omega_{i} N+\sum_{j} \omega_{i j} Y_{j}+\sum_{j, r} B_{i j}^{r} \omega_{j} \xi_{r}, \\
d \xi_{r} & =-\sum_{i} C_{i}^{r} \omega_{i} Y-\sum_{i, j} \omega_{i} B_{i j}^{r} Y_{j}+\sum_{s} \theta_{r s} \xi_{s},
\end{aligned}
$$


where $\omega_{i j}$ denotes the connection 1-forms of the Möbius metric $g$ and $\theta_{r s}$ are the normal connection 1-forms. The tensors

$$
\mathbf{A}=\sum_{i, j} A_{i j} \omega_{i} \otimes \omega_{j}, \quad \mathbf{B}=\sum_{i, j, r} B_{i j}^{r} \omega_{i} \otimes \omega_{j} \xi_{r}, \Phi=\sum_{j, r} C_{j}^{r} \omega_{j} \xi_{r}
$$

are called the Blaschke tensor, the Möbius second fundamental form and the Möbius form of $\varphi$, respectively (see [134]).

The integrability conditions for the structure equations are given by

$$
\begin{aligned}
& A_{i j, k}-A_{i k, j}=\sum_{r}\left(B_{i k}^{r} C_{j}^{r}-B_{i j}^{r} C_{k}^{r}\right), \\
& C_{i, j}^{r}-C_{j, i}^{r}=\sum_{k}\left(B_{i k}^{r} A_{k j}-B_{j k}^{r} A_{k i}\right), \\
& B_{i j, k}^{r}-B_{i k, j}^{r}=\delta_{i j} C_{k}^{r}-\delta_{i k} C_{j}^{r}, \\
& R_{i j k l}=\sum_{r}\left(B_{i k}^{r} B_{j l}^{r}-B_{i l}^{r} B_{j k}^{r}\right)+\delta_{i k} A_{j l}+\delta_{j l} A_{i k}-\delta_{i l} A_{j k}-\delta_{j k} A_{i l}, \\
& R_{r s i j}^{\perp}=\sum_{k}\left(B_{i k}^{r} B_{k j}^{s}-B_{i k}^{s} B_{k j}^{r}\right) .
\end{aligned}
$$

Here the covariant derivatives $A_{i j, k}, B_{i j, k}^{r}, C_{i, j}^{r}$ are defined as usual and $R$ and $R^{\perp}$ are the the curvature tensor of $\mathrm{g}$ and the normal curvature tensor, respectively. The tensor $\mathbf{B}$ satisfies the following identities

$$
\sum_{j} B_{j j}^{r}=0, \quad \sum_{i, j, r}\left(B_{i j}^{r}\right)^{2}=4 .
$$

All coefficients in the structure equations are determined by $\{\mathrm{g}, \mathbf{B}\}$ and the normal connection $\left\{\theta_{\alpha \beta}\right\}$. These are the complete set of Möbius invariants given by Changping Wang derived in [134].

\subsection{Wintgen ideal submanifolds with a two-dimensional integrable distribution}

For a Wintgen ideal submanifold $M^{n}$ in a real-space form $R^{m}(c)$, let $\mathfrak{D}_{2}$ be the 2-dimensional distribution defined by (7.5).

In [91], T. Li, X. Ma and C. Wang proved the following two classification theorems.

Theorem 14.1. Let $\phi: M^{n} \rightarrow \mathbb{E}^{n+p}, n \geq 3$, be a Wintgen ideal submanifold with integrable canonical distribution $\mathfrak{D}_{2}=\operatorname{Span}\left\{e_{1}, e_{2}\right\}$. Then, $\phi$ is locally Möbius equivalent to one of the following:

(a) a cone over a minimal Wintgen ideal surface in the sphere $S^{2+p}$;

(b) or a rotational submanifold over a minimal Wintgen ideal surface in hyperbolic space $H^{2+p}$;

(c) or a cylinder over a minimal Wintgen ideal surface in $\mathbb{E}^{2+p}$.

In the case where the canonical distribution $D_{2}$ is not integrable, T. Li, X. Ma and C. Wang considered the 3-dimensional distribution $\mathfrak{D}_{3}=\operatorname{Span}\left\{e_{1}, e_{2},\left[e_{1}, e_{2}\right]\right\}$ and obtained the following.

Theorem 14.2. Let $\phi: M^{n} \rightarrow \mathbb{E}^{n+p}, n \geq 4$, be a Wintgen ideal submanifold. If $\mathfrak{D}_{2}$ is not integrable, but $\mathfrak{D}_{3}$ is integrable, then $\phi$ is locally Möbius equivalent to one of the following:

(1) a cone over a 3-dimensional minimal Wintgen ideal submanifold in $S^{3+p}$; or

(2) a rotational submanifold over a 3-dimensional minimal Wintgen ideal submanifold in $\mathrm{H}^{3+p}$; or

(3) a cylinder over a 3-dimensional minimal Wintgen ideal submanifold in $\mathbb{E}^{3+p}$.

\subsection{Mean curvature spheres and Wintgen ideal submanifolds of codimension two}

Put

$$
Q_{+}^{n+2}=\left\{[Z] \in C P^{n+3}:\langle Z, Z\rangle=0,\langle Z, \bar{Z}\rangle>0\right\}
$$

be in the complex quadric $Q^{n+2}$ of a complex projective $(n+3)$-space $C P^{n+3}$.

Let $M^{n}$ be a submanifold of a round $(n+p)$-sphere $S^{n+p}$. By definition, the mean curvature sphere at one point $x \in M^{n}$ is the unique round $n$-sphere tangent to $M^{n}$ at $x$ which shares the same mean curvature vector with $M^{n}$ at $x$. In the codimension two case, this assigns a (oriented) space-like 2-space $\operatorname{Span}_{\mathbf{R}}\left\{\xi_{1}, \xi_{2}\right\}$ in the Lorentzian space $L^{n+4}=\mathbb{E}_{1}^{4}$ which is also identified with the isotropic complex line $\operatorname{Span}_{\mathbf{C}}\left\{\xi_{1}-i \xi_{2}\right\} \in C P^{n+3}$ (with respect to the $\mathrm{C}$-linear extension of the Lorentz metric). When the base point $x$ varies along $M^{n}$, this gives the mean curvature sphere congruence, which is also represented as a Gauss map (see [92] for details)

$$
[\xi] \triangleq\left[\xi_{1}-i \xi_{2}\right]: M^{n} \rightarrow Q_{+}^{n+2}
$$


which is called the conformal Gauss map (see [92]).

A key observation made in [92] is that under the hypothesis of being Wintgen ideal, this $n$-sphere congruence is indeed a 2-parameter family, and its envelope not only recovers $M^{n}$, but also extends it to a submanifold as a sphere bundle over a Riemann surface $\bar{M}^{2}$ (a holomorphic curve). The underlying surface $\bar{M}^{2}$ comes from the quotient surface $\bar{M}^{2}=M^{n} / \Gamma$ (at least locally), where $\Gamma$ is the foliation of $M^{n}$ by the integral submanifolds of the distribution $\mathfrak{D}^{\perp}=\operatorname{Span}\left\{e_{3}, \ldots, e_{n}\right\}$. Moreover, the mean curvature sphere $\left[\xi_{1}-i \xi_{2}\right]$ determines a holomorphic, 1-isotropic curve in $Q_{+}^{n+2}$, and all codimension two Wintgen ideal submanifolds can be constructed by such curves in $Q_{+}^{n+2}$.

T. Li, X. Ma, C. Wang and Z. Xie proved the following main result of [92] which describes Wintgen ideal submanifold of codimension two via holomorphic isotropic curves in $Q_{+}^{n+2}$.

Theorem 14.3. The mean curvature spheres $[\xi] \triangleq\left[\xi_{1}-i \xi_{2}\right] \in Q_{+}^{n+2}$ of a Wintgen ideal submanifold of codimension two is a holomorphic and 1-isotropic curve, i.e.,

$$
\xi_{\bar{z}} \| \xi,\left\langle\xi_{z}, \xi_{z}\right\rangle=0
$$

Conversely, given a holomorphic isotropic curve

$$
[\xi]: \bar{M}^{2} \rightarrow Q_{+}^{n+2} \subset C P^{n+3}
$$

the envelope $\widehat{M}^{n}$ of the corresponding 2-parameter family spheres is an n-dimensional Wintgen ideal submanifold (at the regular points).

T. Li, X. Ma, C. Wang and Z. Xie also pointed out in [92] that, via a complex stereographic projection, their characterization of Wintgen ideal submanifolds given above is equivalent to M. Dajczer and R. Tojeiro's description of such submanifolds in terms of minimal surfaces in the Euclidean space given in [60].

Remark 14.1. Several nice properties of mean curvature spheres of Wintgen ideal surfaces were also obtained by B. Rouxel in [115] (see also [41, Theorems 2.1-2.3]).

\subsection{Möbius homogenous surfaces in $S^{4}$}

Let $x, y, z$ be the natural coordinates of $\mathbb{E}^{3}$ and $u_{1}, \ldots, u_{5}$ that of $\mathbb{E}^{5}$. The mapping defined by

$$
u_{1}=\frac{y z}{\sqrt{3}}, u_{2}=\frac{x z}{\sqrt{3}}, u_{3}=\frac{x y}{\sqrt{3}}, u_{4}=\frac{x^{2}-y^{2}}{2 \sqrt{3}}, u_{5}=\frac{1}{6}\left(x^{2}+y^{2}-2 z^{2}\right)
$$

gives rise to an isometric immersion of $S^{2}\left(\frac{1}{3}\right)$ of curvature $\frac{1}{3}$ into $S^{4}(1)$. Two points $(x, y, z)$ and $(-x,-y,-z)$ of $S^{2}\left(\frac{1}{3}\right)$ are mapped into the same point. Thus, this mapping defines an embedding of the real projective plane $R P^{2}\left(\frac{1}{3}\right)$ into $S^{4}(1)$, known as the Veronese surface.

A submanifold $\phi: M^{n} \rightarrow S^{n+p}$ is called a locally Möbius homogeneous submanifold if for any two points $x, y \in$ $M^{n}$ there exists a Möbius transformation $\gamma \in \mathcal{M}\left(S^{n+p}\right)$ which takes $\phi(x)$ to $\phi(y)$ and takes a neighborhood $U$ of $\phi(x)$ in $\phi\left(M^{n}\right)$ to a neighborhood $V$ of $\phi(y)$ in $\phi\left(M^{n}\right)$.

In [135], C. Wang and Z. Xie obtained a complete classification of the Möbius homogenous surfaces in $S^{4}$ given as follows.

Theorem 14.4. Any locally Möbius homogeneous surface in $S^{4}$ is Möbius equivalent to an open part of one of the following Möbius homogenous surfaces:

(1) a round 2-sphere in $S^{4}$;

(2) the Veronese surface in $S^{4}$;

(3) the inverse of the stereographic projection of the surface $\left\{(\sin v, \cos v, a v, u):(u, v) \in \mathbb{E}^{2}\right\} \subset \mathbb{E}^{4}, a \in \mathbf{R}$;

(4) the inverse of the stereographic projection of the surface

$$
\left\{e^{\epsilon_{1} u+\epsilon_{2} v}\left(a \sin \left(\iota_{1} u+\iota_{2} v\right), a \cos \left(\iota_{1} u+\iota_{2} v\right), b \sin \left(\delta_{1} u+\delta_{2} v\right), b \cos \left(\delta_{1} u+\delta_{2} v\right)\right):(u, v) \in \mathbb{E}^{2}\right\} \subset \mathbb{E}^{4},
$$

where $\epsilon_{i}, \iota_{i}, \delta_{i}(i=1,2)$ and $a, b$ are constants such that $a^{2}+b^{2}=1$ and $\left(\epsilon_{1}, \iota_{1}, \delta_{1}\right)$ is not parallel to $\left(\epsilon_{2}, \iota_{2}, \delta_{2}\right)$;

(5) the inverse of the stereographic projection of the surface $\left\{\left(a e^{-r u} \sin u, a e^{-r u} \operatorname{cosu}, b e^{-r u}, v\right)(u, v) \in \mathbb{E}^{2}\right\} \subset \mathbb{E}^{4}$ where $r, a, b$ are constants which satisfy $r \neq 0$ and $a^{2}+b^{2}=1$. 


\subsection{Wintgen ideal submanifolds with vanishing Möbius form}

In [138] Z. Xie studied Wintgen ideal submanifolds with vanishing Möbius form and obtained the following two classification theorems.

Theorem 14.5. Let $\phi: M^{n} \rightarrow S^{n+p}(n \geq 3, p \geq 1)$ be an umbilical-free Wintgen ideal submanifold. If its Möbius form vanishes, then $\phi$ is locally Möbius equivalent to one of the following:

(1) a cone over a Veronese surface in $S^{2 k}(k \geq 2)$; or

(2) a cone over a flat super-minimal surface in $S^{2 k+1}(k \geq 2)$; or

(3) a cone over a flat super-minimal surface in $S^{3+p}$.

Theorem 14.6. Let $\phi: M^{n} \rightarrow S^{n+2}(n \geq 3)$ be a umbilical-free Wintgen ideal submanifold. If its Möbius form vanishes", then $\phi$ is locally Möbius equivalent to

(a) a cone over the Veronese surface in $S^{4}$; or

(b) a cone over the 3-dimensional minimal Wintgen ideal submanifold

$$
x: S O(3) \rightarrow S^{5} ; \quad(u, v, u \times v) \mapsto \frac{1}{\sqrt{2}}(u, v),
$$

which can also be seen as coming from Hopf bundle over the Veronese surface in $C P^{2}$.

\subsection{Conformal Gauss map and Möbius geometry of Wintgen ideal submanifolds}

Let $\phi: M^{n} \rightarrow S^{n+p}$ be a submanifold of $S^{n+p}$. Via the light-cone model of Möbius geometry, the submanifold $\phi: M^{n} \rightarrow S^{n+p}$ can be lifted to a submanifold in the Lorentz space $\mathbb{E}_{1}^{n+p+2}$ of dimension $n+p+2$; at the same time, the normal bundle $\operatorname{Span}\left\{\hat{\xi}_{1}, \ldots, \hat{\xi}_{p}\right\}$, corresponds to $\operatorname{Span}\left\{\xi_{1}, \ldots, \xi_{p}\right\}$ where $\left\{\xi_{r}\right\}$ are orthonormal space-like vectors in $\mathbb{E}_{1}^{n+p+2}$. The mapping

$$
\Xi: \bar{M}^{2} \rightarrow \operatorname{Gr}\left(p, \mathbb{E}_{1}^{n+p+2}\right)
$$

from $x \in M^{n}$ to the space-like subspace $\operatorname{Span}\left\{\xi_{1}(x), \ldots, \xi_{p}(x)\right\}$ is called the conformal Gauss map of $\phi$.

For the conformal Gauss map of a Wintgen ideal submanifold $\phi: M^{n} \rightarrow S^{n+p}$, X. Ma and Z. Xie proved the following two results in [95].

Theorem 14.7. For a Wintgen ideal submanifold $\phi: M^{n} \rightarrow S^{n+p}$ of dimension $n \geq 3$, the conformal Gauss map $\Xi$ factors as a projection map $\pi: M^{n} \rightarrow \bar{M}^{2}$ (which is a Riemannian submersion up to a constant), and a super-conformal harmonic map from a Riemann surface $\Xi: \bar{M}^{2} \rightarrow \operatorname{Gr}\left(p, \mathbb{E}_{1}^{n+p+2}\right)$. In other words, $\Xi\left(M^{n}\right)$ is a super-minimal surface $\bar{M}^{2} \subset \operatorname{Gr}\left(p, \mathbb{E}_{1}^{n+p+2}\right)$ (endowed with the induced metric).

Theorem 14.8. For a Wintgen ideal submanifold $\varphi: M^{n} \rightarrow S^{n+p}$ and the envelope $\widehat{M}^{n}$, we have the following conclusions:

(1) There is a fiber bundle structure $S^{n-2} \rightarrow \widehat{M}^{n} \rightarrow \bar{M}^{2}$ over a Riemann surface. The fibers are all round spheres of the ambient space.

(2) The projection $\pi: \widehat{M}^{n} \rightarrow \bar{M}^{2}$ is a Riemannian submersion up to a constant.

(3) As a natural extension of $M^{n}, \widehat{M}^{n}$ is still a Wintgen ideal submanifold.

\subsection{Classification of Wintgen ideal submanifolds via Möbius geometry}

Via Möbius geometry, Z. Xie, T. Li, X. Ma and C. Wang gave in [141] a coarse classification of all Wintgen ideal submanifolds into three classes:

Class 1. The reducible cases: $M^{n}$ is a cone, a cylinder or a rotational submanifold over a low dimensional minimal Wintgen ideal submanifold $\hat{M}_{\ell}$ in the space form $S^{\ell+p}, \mathbb{E}^{\ell+p}$ or $H^{\ell+p}$, respectively.

Class 2. The irreducible minimal cases: $M^{n}$ is Möbius equivalent to a minimal Wintgen ideal submanifold in a space form; at the same time it is not of Class 1.

Class 3. The generic case: $M^{n}$ is neither Möbius equivalent to a minimal Wintgen ideal submanifold nor reducible to such an example of lower dimension. 
Consequently, the investigation of Wintgen ideal submanifolds is reduced to the study of minimal ones and the generic (irreducible) ones.

In [141], Z. Xie, T. Li, X. Ma and C. Wang also obtained the following reduction theorem.

Theorem 14.9. Let $\phi: M^{n} \rightarrow \mathbb{E}^{n+p}(n \geq 3, p \geq 2)$ be a Wintgen ideal submanifold without umbilical points and let $\mathfrak{D}$ denote the minimal integrable distribution containing $\mathfrak{D}_{2}$. If $\operatorname{dim} \mathfrak{D}=\ell$, then $\phi$ is locally Möbius equivalent to

(a) a cone over a $\ell$-dimensional minimal Wintgen ideal submanifold in $S^{\ell+p}$;

(b) or a submanifold of revolution over a $\ell$-dimensional minimal Wintgen ideal submanifold in $H^{\ell+p}$;

(c) or a cylinder over a $\ell$-dimensional minimal Wintgen ideal submanifold in $\mathbb{E}^{\ell+p}$.

\section{Symmetry of Wintgen ideal submanfiolds}

\subsection{Deszcz's pseudo-symmetry}

Let us assume that $M^{n}$ is an Riemannian $n$-manifold with metric tensor $g$. The endomorphism $X \wedge Y$ of the Lie algebra of vector fields of $M^{n}$ is defined by

$$
(X \wedge Y) Z=g(Y, Z) X-g(X, Z) Y
$$

for vector fields $X, Y \in \mathfrak{X}\left(M^{n}\right)$, where $\mathfrak{X}(M)$ is Lie algebra of vector fields on $M^{n}$. Let $\nabla$ denote the Levi-Civita connection of $\left(M^{n}, g\right)$. Then

$$
R(X, Y)=\nabla_{X} \nabla_{Y}-\nabla_{Y} \nabla_{X}-\nabla_{[X, Y]}
$$

is called the curvature operator of $\left(M^{n}, g\right)$.

A Riemannian manifold $\left(M^{n}, g\right)$ is called semi-symmetric if, for all vector fields $X, Y \in \mathfrak{X}\left(M^{n}\right)$, we have

$$
R \cdot R=0,
$$

in which the curvature operator $R$ on the left-hand side of (15.2) acts as a derivation on the second $R$.

In [69], R. Deszcz generalized the concept of semi-symmetry and introduced the notion of pseudo-symmetric manifolds as follows: For a $(0, k)$-tensor $T$ on $M^{n}$, the following $(0, k+2)$-tensor $Q(g, T)$ was defined by $\mathrm{S}$. Tachibana in [128].

$$
\begin{aligned}
Q(g, T)\left(X_{1}, \ldots, X_{k} ; X, Y\right) & =((X \wedge Y) \cdot T)\left(X_{1}, \ldots, X_{k}\right) \\
& =-T\left((X \wedge Y) X_{1}, \ldots, X_{k}\right)-\cdots-T\left(X_{1}, \ldots, X_{k-1},(X \wedge Y) X_{k}\right) .
\end{aligned}
$$

A manifold $M^{n}$ is called pseudo-symmetric if its curvature tensor $R$ has the property (see [69])

$$
R \cdot R=L_{R} Q(g, R)
$$

for some function $L_{R}: M^{n} \rightarrow \mathbf{R}$.

Pseudo-symmetric manifolds are natural generalizations of local symmetric and semi-symmetric manifolds. These manifolds arose from studies on totally umbilical submanifolds of semi-symmetric spaces and also from studies on geodesic mappings onto semi-symmetric manifolds. Similarly, a Riemannian manifold $M^{n}(n \geq 3)$ is said to be Ricci pseudo-symmetric in the sense of Deszcz, or simply Deszcz Ricci-symmetric if it satisfies

$$
R \cdot S=L_{S} Q(g, S)
$$

for some function $L_{S}: M^{n} \rightarrow \mathbf{R}$, where $S$ denotes the Ricci tensor of $M^{n}$. Further a Riemannian manifold $M^{n}(n \geq 3)$ is said to have a pseudo-symmetric conformal Weyl tensor $C$ if, in the same notations as before,

$$
C \cdot C=L_{C} Q(g, C)
$$

for some function $L_{C}: M^{n} \rightarrow \mathbf{R}$. 


\subsection{Pseudo-symmetry of Wintgen ideal submanifolds in real space forms}

The following results were proved by S. Decu, M. Petrović-Torgašev, A. Šebeković and L. Verstraelen in [70].

Theorem 15.1. Let $M^{n}(n \geq 4)$ be a Wintgen ideal submanifold codimension 2 in a real space form $R^{n+q}(c)(q \geq 2)$ of constant curvature c. Then $M^{n}$ is Deszcz symmetric if and only if $M^{n}$ is totally umbilical in $R^{n+q}(c)$ in which case $L_{R}=0$, or $M^{n}$ is minimal in $R^{n+q}(c)$ in which case $L_{R}=c$.

Theorem 15.2. Let $M^{n}(n \geq 4)$ be a Wintgen ideal submanifold of a real space form $R^{n+q}(c)(q \geq 2)$. Then $M^{n}$ is Deszcz Ricci-symmetric if and only if $M^{n}$ is Deszcz symmetric.

Theorem 15.3. Every Wintgen ideal submanifold $M^{n}(n \geq 4)$ of a real space form $R^{n+q}(c)(q \geq 2)$ is a Riemannian manifold with pseudo-symmetric conformal Weyl tensor.

Theorem 15.4. Let $M^{n}(n \geq 4)$ be a Wintgen ideal submanifold of a real space form $R^{n+q}(c)(q \geq 2)$. Then $M^{n}$ is minimal if and only if the pseudo-symmetric conformal Weyl tensor $C$ is given by

$$
L_{C}=\frac{n-3}{(n-1)(n-2)}(c-\inf K) .
$$

Remark 15.1. When the codimension of $M^{n}$ is 3 (resp., codimension of $M^{n}$ is 2), Theorems 15.1-15.4 were obtained earlier in [70] (resp., in [112]).

\subsection{Pseudo-psymmetry of Wintgen ideal Lagrangian submanifolds in complex space forms}

Recall that the Wintgen type inequality was established by I. Mihai in [101] for Lagrangian submanifolds in complex space forms (see Theorem 9.2).

In [111], M. Petrović-Torgašev and A. Pantić proved the following three results for Lagrangian submanifolds of complex space forms.

Theorem 15.5. A Wintgen ideal Lagrangian submanifold $M^{n}(n \geq 4)$ of a complex space form $\widetilde{M}^{n}(4 c)$ is a Deszcz symmetric Riemannian manifold if and only if it is totally geodesic (with $L_{R}=0$ ) or a minimal or pseudo-umbilical submanifold of $\widetilde{M}^{n}(4 c)$.

Theorem 15.6. A Wintgen ideal Lagrangian submanifold $M^{n}(n \geq 4)$ of a complex space form $\widetilde{M}^{n}(4 c)$ is a Deszcz symmetric if and only if it is Deszcz Ricci-symmetric.

Theorem 15.7. Let $M^{n}(n \geq 4)$ be a Wintgen ideal Lagrangian submanifold of a complex space form $\widetilde{M}^{n}(4 c)$. Then (1) $M^{n}$ is conformally flat if and only if $M^{n}$ is a totally geodesic submanifold.

(2) If $M^{n}$ is not a conformally flat submanifold, then $M^{n}$ has pseudo-symmetric conformal Weyl tensor $C$ and the corresponding function $L_{C}$ defined by (15.5) is given by

$$
L_{C}=\frac{n-3}{\left(n^{2}-1\right)(n-2)^{2}}(\tau-n(n-1) \inf K),
$$

where $\tau$ is the scalar curvature of $M^{n}$.

15.4. Pseudo-psymmetry of Wintgen ideal Legendrian submanifolds in Sasakian space forms

The Wintgen type inequality for Legendrian submanifolds in Sasakian space forms was established by I. Mihai in [104] (see Theorem 10.1). For such submanifolds, A. Šebeković, M. Petrović-Torgašev and A. Pantić proved the following three results in [120].

Theorem 15.8. A Wintgen ideal Legendrian submanifold $M^{n}(n \geq 4)$ of a Sasakian space form $\widetilde{M}^{2 n+1}(c)$ of constant $\varphi$-sectional curvature $c$ is a Deszcz symmetric Riemannian manifold if and only if it is totally umbilical $\left(\right.$ with $\left.L_{R}=0\right)$ or a minimal or pseudo-umbilical submanifold of $\widetilde{M}^{2 n+1}(c)$ (with $\left.L_{R}=-\frac{c+3}{4}-\|H\|^{2}\right)$.

Theorem 15.9. Any Wintgen ideal Legendrian submanifold $M^{n}(n \geq 4)$ of a Sasakian space form $\widetilde{M}^{2 n+1}(c)$ is a Deszcz symmetric if and only if it is Deszcz Ricci-symmetric. 
Theorem 15.10. Let $M^{n}(n \geq 4)$ be a Wintgen ideal Legendrian submanifold of a Sasakian space form $\widetilde{M}^{2 n+1}(c)$. Then (1) $M^{n}$ is conformally flat if and only if $M^{n}$ is a totally umbilical submanifold of $\widetilde{M}^{2 n+1}(c)$.

(2) If $M^{n}$ is not a conformally flat submanifold, then $M^{n}$ has pseudo-symmetric conformal Weyl tensor $C$ and the corresponding function $L_{C}$ defined by (15.5) is given by

$$
L_{C}=\frac{n-3}{\left(n^{2}-1\right)(n-2)^{2}}(\tau+n(n-1) \inf K)
$$

where $\tau$ is the scalar curvature of $M^{n}$.

\subsection{Roter spaces, Deszcz symmetric spaces, and Wintgen ideal submanifolds}

Let $\left(M^{n}, g\right)$ be a Riemannian $n$-manifold with $n \geq 4$ and Ricci tensor $S$. Put

$$
U_{S}=\left\{x \in M^{n}: S-\frac{\tau}{n} g \neq 0 \text { at } x\right\}
$$

and denote by $U_{C}$ the set of all points of $M^{n}$ at which the Weyl-conformal curvature tensor $C \neq 0$. Then $\left(M^{n}, g\right)$ is called a Roter type manifold or a Roter space if its Riemannian curvature tensor $R$ satisfies

$$
R=\alpha S \wedge S+\beta g \wedge S+\gamma g \wedge g
$$

where $\alpha, \beta, \gamma$ are some functions on $U_{S} \cap U_{C}$.

The following link between Roter spaces, Deszcz symmetric spaces, and Wintgen ideal submanifolds was proved by S. Decu, M. Petrović-Torgašev, A. Šebeković and L. Verstraelen in [67]

Theorem 15.11. Let $M^{n}(n \geq 4)$ be a Wintgen ideal submanifold of a real space form $R^{m}(c)$. Then $M^{n}$ is Deszcz symmetric if and only if $M^{n}$ is a Roter space

\section{References}

[1] Alegre, P., Blair, D. E., Carriazo, A.: Generalized Sasakian space forms. Israel J. Math. 141, 157-183 (2004).

[2] Alodan, H., Chen, B.-Y., Deshmukh, S., Vilcu, G.-E.: A generalized Wintgen inequality for quaternionic CR-submanifolds. Rev. R. Acad. Cienc. Exactas Fis. Nat. Ser. A Mat. 114 (3), Art 129, 14 pp (2020).

[3] Amari, S.: Differential-geometrical methods in statistics. Springer-Verlag, New York, NY (1985).

[4] Anciaux, H.: Minimal submanifolds in pseudo-Riemannian geometry, World Scientific, Hackensack, NJ (2011).

[5] Aquib, M., Lone, M. S., Lone, M. A.: Generalized Wintgen inequality for bi-slant submanifolds in locally conformal Kaehler space forms. Mat. Vesnik 70 (3), 243-249 (2018).

[6] Aquib, M., Shahid, M. H.: Generalized Wintgen inequality for submanifolds in Kenmotsu space forms.Tamkang J. Math. 50 (2), 155-164 (2019).

[7] Aydin, M. E., Mihai, I.: Wintgen inequality for statistical surfaces, Math. Inequal. Appl. 22 (1), 123-132 (2019).

[8] Aydin, M. E., Mihai, A., Mihai, I. : Generalized Wintgen inequality for statistical submanifolds in statistical manifolds of constant curvature. Bull. Math. Sci. 7 (1), 155-166 (2017)

[9] Aytimur, H., Ozgur, C.: Inequalities for submanifolds in statistical manifolds of quasi-constant curvature. Ann. Polon. Math. 121 (3), 197-215 (2018).

[10] Bansal, P., Shahid, M. H., Lone, M. A.: Geometric bounds for $\delta$-Casorati curvature in statistical submanifolds of statistical space forms. Balkan J. Geom. Appl. 24 (1), 1-11 (2019).

[11] Bansal, P., Uddin, S., Shahid, M. H.: On the normal scalar curvature conjecture in Kenmotsu statistical manifolds. J. Geom. Phys. 142, 37-46 (2019).

[12] Barros, M., Chen, B.-Y., Urbano, F.: Quaternion CR-submanifolds of quaternion manifolds. Kodai Math. J. 4, 399-417 (1981).

[13] Bejancu, A.: Geometry of $C R$-submanifolds. D. Reidel Publ. Co., Bodrecht-Boston-Lancaster-London (1986).

[14] Blair, D. E.: Riemannian geometry of contact and symplectic manifolds. Progress in Mathematics, 203, Birkhäuser, Boston, MA (2002).

[15] Borrelli, V., Chen, B.-Y., Morvan, J.-M.: Une caractérisation géométrique de la sphère de Whitney. C. R. Acad. Sci. Paris Sér. I Math. 321, 1485-1490 (1995).

[16] Bulca, B., Arslan, K.: Semiparalel Wintgen Ideal Surfaces in $\mathbb{E}^{n}$, C. R. Acad. Bulgare Sci. 67 (5), 613-622 (2014).

[17] Byrd, P. F., Friedman, M. D.: Handbook of elliptic integrals for engineers and scientists. Second edition, Springer-Verlag, New YorkHeidelberg (1971)

[18] Borůvka, O.: Sur une classe de surfaces minma plonées dans un espace á quatre dimensions à courbure constante. C. R. Acad. Sci. 187, 334-336 (1928).

[19] Boyom, M. N., Jabeen, Z., Lone, M. A., Lone, M. S., Shahid, M. H. Generalized Wintgen inequality for Legendrian submanifolds in Sasakian statistical manifolds. In: Geometric science of information, pp. 407-412, Lecture Notes in Comput. Sci., 11712, Springer, Cham, 2019.

[20] Bryant, R. L. Conformal and minimal immersions of compact surfaces into the 4-sphere. J. Diff. Geom. 17, 455-473 (1982).

[21] Carriazo, A.: Bi-slant immersions. in: Proc. ICRAMS 2000, Kharagpur, India, pp. 88-97 (2000). 
[22] Castro, I.: Lagrangian surfaces with circular ellipse of curvature in complex space forms. Math. Proc. Cambridge. Philo. Soc. 136, 239-245 (2004).

[23] Chen, B.-Y.: Slant immersions. Bull. Austral. Math. Soc. 41, 135-147 (1990).

[24] Chen, B.-Y.: Geometry of Slant Submanifolds. Katholieke Universiteit Leuven, Belgium (1990).

[25] Chen, B.-Y.: Some pinching and classification theorems for minimal submanifolds. Arch. Math. 60, 568-578 (1993).

[26] Chen, B.-Y.: Differential geometry of semiring of immersions I. General theory. Bull. Inst. Math. Acad. Sinica 21 (1), 1-34 (1993).

[27] Chen, B.-Y.: Mean curvature and shape operator of isometric immersions in real-space forms. Glasgow Math. J. 38, 87-97 (1996).

[28] Chen, B.-Y.: Complex extensors and Lagrangian submanifolds in complex Euclidean spaces. Tohoku Math. J. 49, 277-297 (1997).

[29] Chen, B.-Y.: Interaction of Legendre curves and Lagrangian submanifolds. Israel J. Math. 99, 69-108 (1997).

[30] Chen, B.-Y.: Strings of Riemannian invariants, inequalities, ideal immersions and their applications. In: The Third Pacific Rim Geometry Conference (Seoul, 1996), pp. 7-60, Int. Press, Cambridge MA (1998).

[31] Chen, B.-Y.: Relations between Ricci curvature and shape operator for submanifolds with arbitrary codimensions. Glasgow Math. J. 41 (1), $33-41$ (1999).

[32] Chen, B.-Y.: Some new obstructions to minimal and Lagrangian isometric immersions. Japan. J. Math. 26, 105-127 (2000).

[33] Chen, B.-Y.: Riemannian submanifolds. In: Handbook of Differential Geometry. Vol. I, pp. 187-418, North-Holland, Amsterdam (2000).

[34] Chen, B.-Y.: Riemannian geometry of Lagrangian submanifolds. Taiwanese J. Math. 5, 681-723 (2001).

[35] Chen, B.-Y.: $\delta$-invariants, inequalities of submanifolds and their applications. In: Topics in differential geometry, pp. 29-155, Ed. Acad. Romane, Bucharest (2008).

[36] Chen, B.-Y.: Classification of Wintgen ideal surfaces in Euclidean 4-space with equal Gauss and normal curvature. Ann. Global Anal. Geom. 38, $145-160$ (2010).

[37] Chen, B.-Y.: A minimal immersion of hyperbolic plane in neutral pseudo-hyperbolic 4-space and its characterization. Arch. Math. 94, 257-265 (2010).

[38] Chen, B.-Y.: A Wintgen type inequality for surfaces in 4D neutral pseudo-Riemannian space forms and its applications to minimal immersions. JMI Int. J. Math. Sci., 1, 1-12 (2010).

[39] Chen, B.-Y.: Classification of minimal Lorentz surfaces in indefinite space forms with arbitrary codimension and arbitrary index. Publ. Math. Debrecen. 78 (2), 485-503 (2011).

[40] Chen, B.-Y.: Pseudo-Riemannian Geometry, $\delta$-invariants and Applications. World Scientific, Hackensack, NJ (2011).

[41] Chen, B.-Y.: On Wintgen ideal surfaces. In: Riemannian geometry and applications-Proceedings RIGA 2011, pp. 59-74, Ed. Univ. Bucuresti, Bucharest (2011).

[42] Chen, B.-Y.: Wintgen ideal surfaces in four-dimensional neutral indefinite space form $R_{2}^{4}($ c). Results Math. 61, 329-345 (2012).

[43] Chen, B.-Y.: A tour through $\delta$-invariants: from Nash's embedding theorem to ideal immersions, best ways of living and beyond. Inst. Math. Publ. 94(108), 67-80 (2013).

[44] Chen, B.-Y.: On ideal hypersurfaces of Euclidean 4-space. Arab J. Math. Sci. 19, 129-144 (2013).

[45] Chen, B.-Y.: Total mean curvature and submanifolds of finite type. 2nd Edition, World Scientific, Hackensack, NJ (2015).

[46] Chen, B.-Y.: Differential geometry of warped product manifolds and submanifolds. World Scientific, Hackensack, NJ (2017).

[47] Chen, B.-Y.: Recent developments in $\delta$-Casorati curvatures. Turk. J. Math. 45 (1), 1-46 (2021).

[48] Chen, B.-Y., Dillen, F., Verstraelen, L., Vrancken, L.: Totally real submanifolds of $C P^{n}$ satisfying a basic equality. Arch. Math. 63, 553-564 (1994).

[49] Chen, B.-Y., Houh, C. S.: Totally real submanifolds of a quaternion projective space. Ann. Mat. Pura Appl. 70, 185-199 (1979).

[50] Chen, B.-Y., Mihai, A., Mihai, I.: A Chen first inequality for statistical submanifolds in Hessian manifolds of constant Hessian curvature. Results Math. 74 (4), Art. 165, 11 pp. (2019).

[51] Chen, B.-Y., Ogiue, K.: On totally real submanifolds. Trans. Amer. Math. Soc. 193, 257-266 (1974).

[52] Chen, B.-Y., Suceavă, B. D.: Classification theorems for space-like surfaces in 4-dimensional indefinite space forms with index 2. Taiwanese J. Math. 15, 523-541 (2011).

[53] Chen, B.-Y., Yamaguchi, S.: Classification of surfaces with totally geodesic Gauss image. Indiana Univ. Math. J. 32, 143-154 (1983).

[54] Chen, B.-Y., Yamaguchi, S.: Submanifolds with totally geodesic Gauss image. Geom. Dedicata. 15, 313-322 (1984).

[55] Chen, B.-Y., Yano, K.: Hypersurfaces of a conformally flat space. Tensor (N.S.). 26, 318-322 (1972).

[56] Chen, B.-Y., Yano, K.: Special conformally flat spaces and canal hypersurfaces. Tohoku Math. J. 25, 177-184 (1973).

[57] Choudhary,M. A., Bahadir, O., Alsulami, H.: Generalized Wintgen inequality for some submanifolds in golden Riemannian space forms. Balkan J. Geom. Appl. 25 (2), 1-11 (2020).

[58] Crasmareanu, M., Hretcanu, C.: Golden differential geometry. Chaos Solitons \& Fractals. 38 (5), 1229-1238 (2008).

[59] Dajczer, M., Tojeiro, R.: All superconformal surfaces in $\mathbb{R}^{4}$ in terms of minimal surfaces. Math. Z. 261, 869-890 (2009).

[60] Dajczer, M., Tojeiro, R.: Submanifolds of codimension two attaining equality in an extrinsic inequality. Math. Proc. Cambridge Philos. Soc. 146 (2), 461-474 (2009)

[61] DeSmet, P. J., Dillen, F., Verstraelen, L., Vrancken, L.: A pointwise inequality in submanifold theory. Arch. Math. (Brno). 35, 115-128 (1999).

[62] Decruyenaere, F., Dillen, F., Mihai, I., Verstraelen, L.: Tensor products of spherical and equivariant immersions. Bull. Belg. Math. Soc. Simon Stevin. 1 (5), 643-648 (1994).

[63] Decu, S.: Extrinsic and intrinsic principal directions of ideal submanifolds. Bull. Transilv. Univ. Brasov Ser. III. 1(50), $93-97$ (2008).

[64] Decu, S., Haesen, S., Verstraelen, L.: Optimal inequalities involving Casorati curvatures. Bull. Transilvania Univ. of Braşov Series III, Math. Inform. Phys. 14(49), 85-93 (2007).

[65] Decu, S., Haesen, S., Verstraelen, L., Vîlcu, G.-E.: Curvature invariants of statistical submanifolds in Kenmotsu statistical manifolds of constant $\phi$-sectional curvature. Entropy. 20, Art. 529 (2018).

[66] Decu, S., Petrović-Torgašev, M., Šebeković, A., Verstraelen, L.: On the intrinsic Deszcz symmetries and the extrinsic Chen character of Wintgen ideal submanifolds. Tamkang J. Math. 41 (2), 109-116 (2010).

[67] Decu, S., Petrović-Torgašev, M., Šebeković, A., Verstraelen, L.: On the Roter type of Wintgen ideal submanifolds. Rev. Roumaine Math. Pures Appl. 57 (1), 75-90 (2012).

[68] Decu, S., Petrović-Torgašev, M., Šebeković, A., Verstraelen, L.: Ricci and Casorati principal directions of Wintgen ideal submanifolds. Filomat. 28 (4), 657-661 (2014).

[69] Deszcz, R.: On pseudosymmetric spaces. Bull. Soc. Math. Belg. 44, 1-34 (1992). 
[70] Deszcz, R., Petrović-Torgašev, M., Sentürk, Z., Verstraelen, L.: Characterization of the pseudo-symmetries of ideal Wintgen submanifolds of dimension 3. Publ. Inst. Math. (Beograd) (N.S.). 88(102), 53-65 (2010).

[71] Dillen, F., Fastenakels, J., Van der Veken, J.: A pinching theorem for the normal scalar curvature of invariant submanifolds. J. Geom. Phys. 57, 833-840 (2007).

[72] Dillen, F., Fastenakels, J., Van der Veken, J.: Remarks on an inequality involving the normal scalar curvature. In: Pure and applied differential geometry-PADGE 2007, pp. 83-92, Ber. Math., Shaker Verlag, Aachen (2007).

[73] Dragomir, S., Ornea, L.: Locally Conformal Kaehler Geometry. Birkhauser, Basel (1998).

[74] Friedrich, Th.: On surfaces in four-spaces. Ann. Glob. Anal. Geom. 2, 257-287 (1984).

[75] Friedrich, Th.: On superminimal surfaces. Arch. Math. (Brno). 33, 41-56 (1997).

[76] Furuhata, H.: Hypersurfaces in statistical manifolds. Differential Geom. Appl. 27, 420-429 (2009).

[77] Furuhata, H., Hasegawa, I., Okuyama, Y., Sato,K.: Kenmotsu statistical manifolds and warped product. J. Geom. 108, 1175-1191 (2017).

[78] Ge, J., Tang, Z.: A proof of the DDVV conjecture and its equality case. Pacific J. Math. 237, 87-95 (2008).

[79] Gheysens, L., Verheyen, P., Verstraelen, L.: Sur les surfaces A ou les surfaces de Chen. C. R. Acad. Sci. Paris Sér. I Math. 292, 913-916 (1981).

[80] Gheysens, L., Verheyen, P., Verstraelen, L.: Characterization and examples of Chen submanifolds. J. Geom. 20, 47-62 (1983).

[81] Goląb, S.: On semi-symmetric and quarter-symmetric linear connections. Tensor (N.S.). 29 (3), $249-254$ (1975).

[82] Görünüş, R., Erken, I. K., Yazla, A., Murathan, C.: A generalized Wintgen inequality for Legendrian submanifolds in almost Kenmotsu statistical manifolds. Int. Electron. J. Geom. 12 (1), 43-56 (2019).

[83] Guadalupe, I. V., Rodriguez, L.: Normal curvature of surfaces in space forms. Pacific J. Math. 106, 95-103 (1983).

[84] Haesen, S.: Optimal inequalities for embedded space-times. Kragujevac J. Math. 28, 69-85 (2005).

[85] Hoffman, D., Osserman, R.: The geometry of the generalized Gauss map, Mem. Amer. Math. Soc. 236 (1980).

[86] Hui, S. K., Lemence, R. S., Mandal, P.: Wintgen inequalities on Legendrian submanifolds of generalized Sasakian-space-forms. Comment. Math. Univ. Carolin. 61 (1), 105-117 (2020).

[87] Ishihara, S.: Quaternion Kählerian manifolds. J. Differential Geometry. 9, 483-500 (1974).

[88] Kashiwada, T.: Some properties of locally conformally Kaehler manifolds. Hokkaido J. Math. 8, 191-198 (1979).

[89] Kommerell, K.: Rimannsche Flächen im ebenen Raum von vier Dimensionen. Math. Ann. 60, 548-596 (1905).

[90] Kwietniewski, S.: Über Flächen der 4-dimensionalen Raumes deren Tangentialebenen paarweise isoklin sind. Doctoral Dissertation, University of Zürich (1902).

[91] Li, T., Ma, X., Wang, C.: Wintgen ideal submanifolds with a low-dimensional integrable distribution. Front. Math. China. 10 (1), 111-136 (2015).

[92] Li, T., Ma, X., Wang, C., Xie, Z.: Wintgen ideal submanifolds of codimension two, complex curves, and Möbius geometry. Tohoku Math. J. 68 (4), 621-638 (2016).

[93] Loo, B.: The space of harmonic maps of $S^{2}$ into $S^{4}$. Trans. Amer. Math. Soc. 313, 81-102 (1989).

[94] Lu, Z.: Normal scalar curvature conjecture and its applications. J. Funct. Anal. 261, 1284-1308 (2011).

[95] Ma, X., Xie, Z.: The Möbius geometry of Wintgen ideal submanifolds. In: Real and complex submanifolds, pp. 411-425, Springer Proc. Math. Stat., 106, Springer, Tokyo (2014).

[96] Macsim, G., Ghişoiu, V.: Generalized Wintgen inequality for Lagrangian submanifolds in quaternionic space forms. Math. Inequal. Appl. 22 (3), 803-813 (2019).

[97] Matsumoto, K.: Locally conformally Kaehler manifolds and their applications. Mem. Sect. Stiit. Acad. Romania Ser. IV 14, 7-49 (1991).

[98] Mihai, A.: Scalar normal curvature of Lagrangian 3-dimensional submanifolds in complex space forms. In: Pure and applied differential geometry-ADGE 2007, pp. 171-177, Ber. Math., Shaker Verlag, Aachen (2007).

[99] Mihai, A., Mihai, I.: CR-submanifolds in complex and Sasakian space forms. In: Geometry of Cauchy-Riemann submanifolds, pp. 217-266, Springer, Singapore (2016).

[100] Mihai, I.: On generalized Wintgen inequality. In: Riemannian geometry and applications-Proceedings RIGA 2011, pp. 203-208, Ed. Univ. Bucureşti, Bucharest (2011).

[101] Mihai, I.: On the generalized Wintgen inequality for Lagrangian submanifolds in complex space forms. Nonlinear Anal. 95, 714-720 (2014).

[102] Mihai, I.: On the generalized Wintgen inequality for submanifolds in Sasakian space forms. In: Riemannian Geometry and ApplicationsProceedings RIGA 2014, pp. 153-158, Editura Univ. Bucur., Bucharest (2014).

[103] Mihai, I.: On the generalized Wintgen inequality for submanifolds in complex and Sasakian space forms.In: Recent advances in the geometry of submanifolds-dedicated to the memory of Franki Dillen (1963-2013), pp. 111-126, Contemp. Math., 674, Amer. Math. Soc., Providence, RI (2016).

[104] Mihai, I.: On the generalized Wintgen inequality for Legendrian submanifolds in Sasakian space forms. Tohoku Math. J. 69 (1), 43-53 (2017).

[105] Murathan, C., Şahin, B. : A study of Wintgen like inequality for submanifolds in statistical warped product manifolds. J. Geom. 109 (2), Art. 30, 18 pp (2018).

[106] Ogiue, K.: Differential geometry of Kaehler submanifolds. Adv. Math. 13, 73-114 (1974).

[107] Opozda, B.: Bochner's technique for statistical structures. Ann. Glob. Anal. Geom. 48 (4), 357-395 (2015).

[108] Opozda, B.: A sectional curvature for statistical structures. Linear Algebra Appl. 497, 134-161 (2016).

[109] Ou, Y.-L., Chen, B.-Y.: Biharmonic Submanifolds and Biharmonic Maps in Riemannian Geometry. World Scientific, Hackensack, NJ (2020).

[110] Petrović-Torgašev, M.: Deszcz symmetries of ideal submanifolds. Bull. Transilv. Univ. Braşov Ser. B (N.S.). 14(49), suppl., 249-262 (2007).

[111] Petrović-Torgašev, M., Pantić, A.: Pseudo-symmetries of generalized Wintgen ideal Lagrangian submanifolds. Publ. Inst. Math. (Beograd) (N.S.) 103(117), 181-190 (2018).

[112] Petrović-Torgašev, M., Verstraelen, L.: On Deszcz symmetries of Wintgen ideal submanifolds. Arch. Math. (Brno). 44, 57-67 (2008).

[113] Ö. Poyraz, N., Yaşar, E.: Lightlike hypersurfaces of a golden semi-Riemannian manifold. Mediterr. J. Math. 14 (5) Paper No. 204, 20 pp (2017).

[114] Reckziegel, H.: Horizontal lifts of isometric immersions into the bundle space of a pseudo-Riemannian submersion. In: Global Diff. Geom and Global Analysis (1984), Lecture Notes in Mathematics 12, pp. 264-279 (1985).

[115] Rouxel, B.: Harmonic spheres of a submanifold in Euclidean space. In: Proc. Proceedings of the 3rd Congress of Geometry (Thessaloniki, 1991), pp. 357-364, Aristotle Univ. Thessaloniki, Thessaloniki (1992).

[116] Rouxel, B.: Chen submanifolds. In: Geometry and topology of submanifolds, VI (Leuven, 1993/Brussels, 1993), pp. 185-198, World Sci. Publ., River Edge, NJ (1994).

[117] Sasaki, M.: Space-like maximal surfaces in 4-dimensional space forms of index 2. Tokyo J. Math. 25, 295-306 (2002). 
[118] Schouten, J. A., van Kampen, E. R. : Zur Einbettungs- und Krümmungstheorie nichtholonomer Gebilde. Math. Ann. 103, 752-783 (1930).

[119] Šebeković, A.: Symmetries of Wintgen ideal submanifolds. Bull. Transilv. Univ. Braşov Ser. III 1(50), 333-341 (2008).

[120] Šebeković, A., Petrović-Torgašev, M., Pantić, A.: Pseudosymmetry properties of generalised Wintgen ideal Legendrian submanifolds, Filomat. 33 (4), 1209-1215 (2019).

[121] Sentürk, Z.: Characterisation of the Deszcz symmetric ideal Wintgen submanifolds. An. Ştiinţ. Univ. Al. I. Cuza Iaşi. Mat. (N.S.). 53, suppl. 1, 309-316 (2007).

[122] Şahin, B.: Slant submanifolds of quaternion Kaehler manifolds. Comm. Korean Math. Soc. 22 (1), 23-35 (2007).

[123] Shima, H.: The geometry of Hessian structures. World Scientific, Hackensack, NJ (2007).

[124] Siddiqui, A. N., Ahmad, K.: Generalized Wintgen inequality for totally real submanifolds in LCS-manifolds. Balkan J. Geom. Appl. 24 (2), 53-62 (2019).

[125] Siddiqui, A. N., Al-Solamy, F. R., Shahid, M. H., Mihai, I.: On CR-statistical submanifolds of holomorphic statistical manifolds. arXiv:2009.12055v1 [math.DG], 25 September 2020.

[126] Siddiqui, A. N., Chen, B.-Y., Bahadir, O.: Statistical solitons and inequalities for statistical warped product submanifolds, Mathematics. (7), Art. 797, 19 pp (2019).

[127] Siddiqui, A. N., Shahid, M. H.: On totally real statistical submanifolds. Filomat. 32 (13), 4473-4483 (2018).

[128] Tachibana, S.-I.: A theorem on Riemannian manifolds of positive curvature operator. Proc. Japan Acad. 50, 301-302 (1974).

[129] Tanaka, N.: On non-degenerate real hypersurfaces, graded Lie algebras and Cartan connections. Japan J. Math. 2 (1), 131-190 (1976).

[130] Tanno, S.: Variational problems on contact Riemannian manifolds. Trans. Amer. Math. Soc. 314, 349-379 (1989).

[131] Todjihounde, L.: Dualistic structures on warped product manifolds. Diff. Geom. Dyn. Syst. 8, 278-284 (2006).

[132] Vaisman, I.: On locally conformal almost Kaehler manifolds. Israel J. Math. 24, 338-351 (1976).

[133] Vos, P. W.: Fundamental equations for statistical submanifolds with applications to the Bartlett correction. Ann. Inst. Stat. Math. 41, 429-450 (1989).

[134] Wang, C.: Möbius geometry of submanifolds in $S^{n}$. Manuscripta Math. 96, 517-534 (1998).

[135] Wang, C., Xie, Z.: Classification of Möbius homogenous surfaces in $S^{4}$. Ann. Glob. Anal. Geom. 46, 241-257 (2014).

[136] Webster, S. M.: Pseudo-Hermitian structures on a real hypersurface. J. Differential Geometry 13 (1), 25-41 (1978).

[137] Wintgen, P.: Sur l'inégalité de Chen-Willmore. C. R. Acad. Sci. Paris 288, 993-995 (1979).

[138] Xie, Z.: Wintgen ideal submanifolds with vanishing Möbius form, Ann. Global Anal. Geom. 48 (4), 331-343 (2015).

[139] Xie, Z.: Three special classes of Wintgen ideal submanifolds, J. Geom. Phys. 114, 523-533 (2017).

[140] Xie, Z., Li, T., Ma, X., Wang, C.: Möbius geometry of three-dimensional Wintgen ideal submanifolds in $S^{5}$. Sci. China Math. 57 (6), $1203-1220$ (2014).

[141] Xie, Z., Li, T., Ma, X., Wang, C.: Wintgen ideal submanifolds: reduction theorems and a coarse classification. Ann. Global Anal. Geom. 53 (3), 377-403 (2018).

\section{Affiliations}

BANG-YEN CHEN

ADDRESS: Michigan State University, Department of Mathematics, East Lansing, Michigan 48824, U.S.A.

E-MAIL: chenb@msu.edu

ORCID ID: 0000-0002-1270-094X 